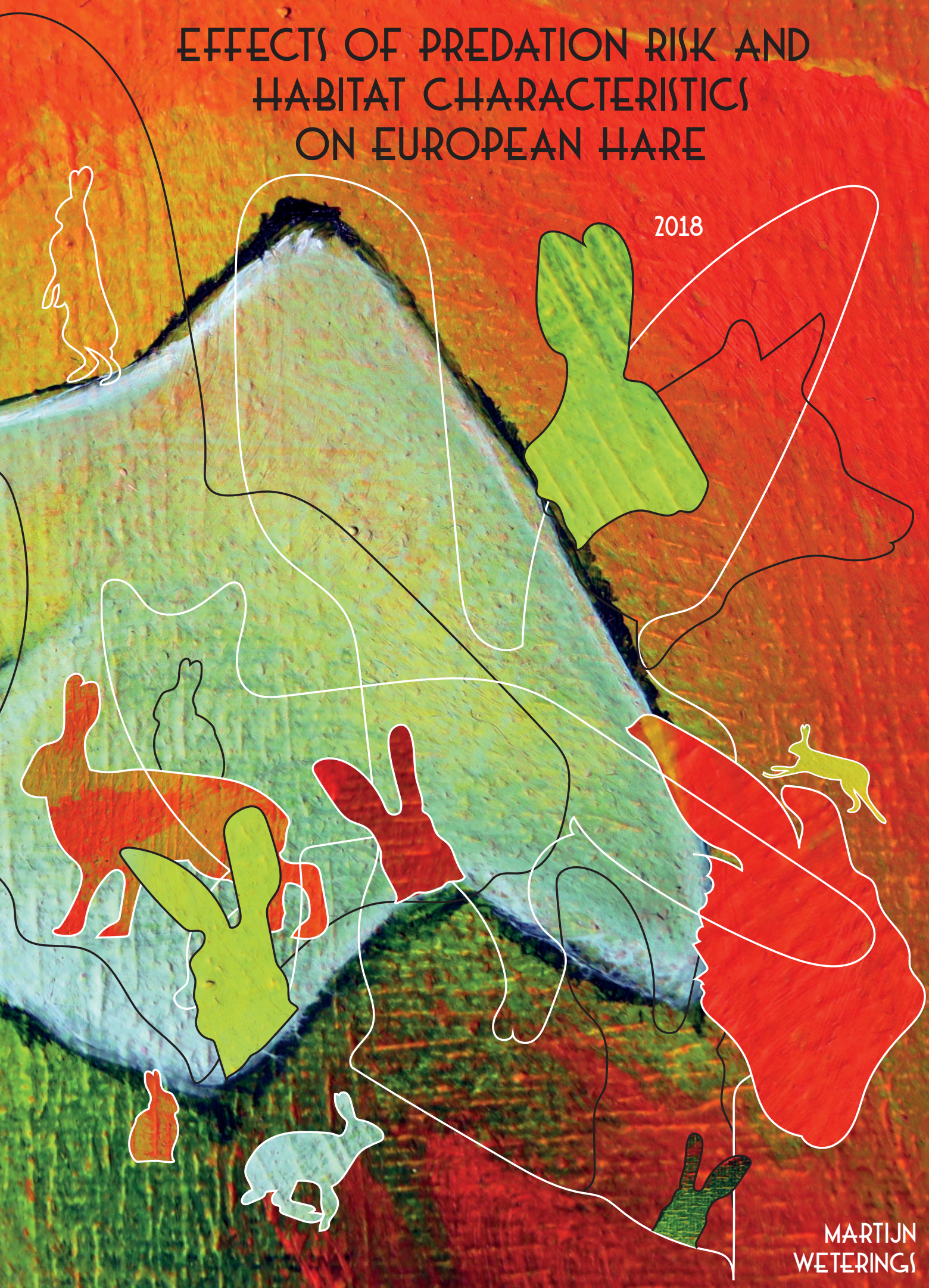





\title{
Effects of predation risk and habitat characteristics on European hare
}

\author{
Martijn J.A. Weterings
}

"The wandering hares are the most dangerous to follow, for they are so cunning in the ways and mazes of the fields, running up the hills and rocks, because by custom they know a nearer way with other tricks to the confusion of the dogs and discouragement of the hunters"

Noble (1800): the sportsman's dictionary; or the gentleman's companion for town and country 


\section{Thesis committee}

\section{Promotor}

Prof. Dr H.H.T Prins

Professor of Resource Ecology

Wageningen University \& Research

\section{Co-promotors}

Dr F. van Langevelde

Associate professor, Resource Ecology Group

Wageningen University \& Research

Dr S.E. van Wieren

\section{Other members}

Prof. Dr K. Hackländer, University of Natural Resources and Life Sciences, Vienna, Austria

Prof. Dr M. Naguib, Wageningen University \& Research

Dr L. Bakker, Netherlands Institute of Ecology, Wageningen

Prof. Dr H.G. Rödel, Paris University, France

This research was conducted under the auspices of the Graduate School Production Ecology \& Resource Conservation 


\title{
Effects of predation risk and habitat characteristics on European hare
}

\author{
Martijn J.A. Weterings
}

Thesis

submitted in fulfilment of the requirements for the degree of doctor at Wageningen University

by the authority of the Rector Magnificus, Prof. Dr A.P.J. Mol, in the presence of the

Thesis Committee appointed by the Academic Board to be defended in public on Thursday 24 May 2018 at 11 a.m. in the Aula. 
Martinus J.A. Weterings

Effects of predation risk and habitat characteristics on European hare, 183 pages.

PhD thesis, Wageningen University, Wageningen, the Netherlands (2018) With references, with summaries in English and Dutch

ISBN: 978-94-6343-868-1

DOI: https://doi.org/10.18174/447195 


\section{TABLE OF CONTENT}

$\begin{array}{ll}\text { Chapter 1: } & \text { General introduction }\end{array}$

Chapter 2: Strong reactive movement response of the medium-sized

European hare to elevated predation risk in short vegetation

Chapter 3: Predators, fellow prey and risk factors: Implications for the space use of two sympatric leporids

Chapter 4: Food quality and quantity is more important explaining foraging of an intermediate-sized mammalian herbivore than predation or competition

Chapter 5: Chronic exposure to high predation risk of multiple predators decreases body condition and reproductive output of European hare

Chapter 6: General discussion

Bibliography

Summary

Samenvatting

Acknowledgements

Short biography

Publications

Affiliation of co-authors

PE\&RC Training and Education Statement 



\section{CHAPTER 1}

General introduction

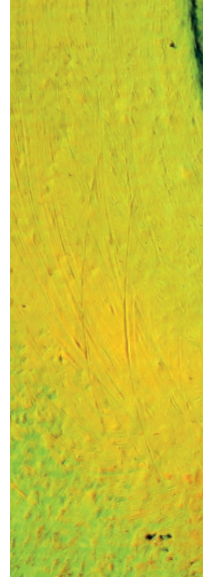





\section{THE CHANGE IN THE LANDSCAPE OF NORTH-WESTERN EUROPE}

Since the 1940s, the landscape in north-western Europe changed, reducing habitat for many species because of modern agricultural practises and a loss of associated habitat diversity, structure and quality (Robinson \& Sutherland, 2002). This general change was caused by an increase in mechanization, removals of hedgerows, increased use of herbicides (Edwards et al., 2000), pesticides (Geiger et al., 2009) and nutrients (Ceulemans et al., 2011), and a decrease in the use of crop rotations. Additionally, 'habitat drainage, changes in soil nutrient status, changes in the timing of cereal sowing and the application of chemicals' (Robinson \& Sutherland, 2002, p.165) affected the number and diversity of plant species (Ceulemans et al., 2011). Because more than $59 \%$ of the total land surface in north-western Europe (BE, DK, GE, LU, NL \& UK) is attributed to agriculture (FAO, 2010), intensification resulted in an accelerated homogenisation of the landscape (Benton et al., 2003). This homogenisation of the landscape possibly improved the accessibility for generalist predators in the landscape, with an increased impact on prey populations (Schneider, 2001; Smith et al., 2005; Gorini et al., 2012). Additionally, predator numbers have increased in north-western Europe in the last decades (e.g., birds of prey: Parlevliet, 2003; red fox: Tapper, 1992; Knauer et al., 2010), while they have also expanded their distribution (e.g., birds of prey: Boele et al., 2008; Hustings \& Vergeer, 2002; red fox: Davidson et al., 2012). However, the effects of predation are often difficult to investigate or not taken into account in explaining prey response to changes in the environment (Jonker et al., 2010).

\section{PREDATION RISK}

Predators affect prey mortality and behaviour (Lima \& Dill, 1990; Ylönen et al., 1992). For example, after the introduction of wolves in Yellowstone National Park, elk populations decreased in numbers (White \& Garrot, 2005). At the same time, elk increased their vigilance (Laundré et al., 2001) and changed their movements depending on the habitat characteristics (Fortin et al., 2005). Elk avoided open areas with a higher predation risk and spent more time in closed habitats of lower risk, reducing foraging efficiency (Winnie et al., 2006). As a response to wolf predation risk, the change in habitat use by elk probably affected the plant species communities (Ripple et al., 2001; Ripple \& Beschta, 2012; but see Kauffman et al., 2010; Mech, 2010). Generally, prey species experience a landscape of fear (Laundré et al., 2001) as is illustrated by the wolf-elk example. As a result, prey anti-predator behaviour can affect prey habitat use, foraging efficiency and 
resource use (Verdolin, 2006; Møller, 2008), having a potential important effect on the food quality and quantity exploited by prey (Shipley, 2007).

Besides habitat characteristics (Gorini et al., 2012), traits of the predators (Creel, 2011) and prey (Sinclair \& Krebs, 2002) and the presence of interspecific competitors (Holt, 2009) (henceforth "competitors") are important factors that determine the response of prey to predation risk. The effects of predation risk, for example, depend on the hunting mode of predators (Creel, 2011), group size and body mass of prey species, or the ability of prey to make use of a refuge for escape (Lima \& Dill, 1990). The population sizes of smaller prey species and solitary prey species are expected to be stronger affected by predation, whereas the larger prey species or prey species that live in groups are expected to be stronger affected by food availability (Sinclair et al., 2003; Hopcraft et al., 2010; Creel, 2011). Very few prey species, however, are affected by one single predator species only (Sih et al., 1998). Grizzly bears in Yellowstone National Park, for example, competed with the introduced wolves over elk prey, causing changes in the timing of predation and the type of elk prey targeted by both predator species (Griffin et al., 2011).

The relative importance and the role of predators in ecosystems for prey is still subject of debate (Smith et al., 2003; Dobson, 2014). In general, risk effects that trigger anti-predator behaviour are at least as strong as the effects of direct consumption (i.e., kills) (Preisser et al., 2005). Predators can affect the physiology (Creel et al., 2007; Creel \& Christianson, 2008) and reproduction (Krebs et al., 2001; Sheriff et al., 2009) of prey species. The introduction of wolves in Yellowstone National Park was correlated with a reduction in elk numbers, much more than could be contributed to prey kills (Vucetich et al., 2005; White \& Garrott, 2005). Additionally calf recruitment of elk was thought to be negatively affected by the physiological cost of the anti-predator behaviour of elk (Creel et al., 2007). Preisser et al. (2005) attributed 58\% of the magnitude of the total predator effect to risk effects of predators, rising to $85 \%$ when also considering cascading effects of predators on resources of prey (respectively $4 \%$ and 52\% larger than effects of direct consumption). However the relative strength of top-down (i.e., predation) versus bottom-up (i.e., food quality and quantity) modulation of prey species behaviour, for example such as in Yellowstone National Park, is still unclear (Dobson, 2014). An understanding of the relative strength of these mechanisms that modulate prey behaviour will also contribute to an improved understanding of the responses of prey species to the changes in the north-west European landscape. Dobson (2014) argued that the key scientific challenge for the $21^{\text {th }}$ 
century is to understand the complex interactions between predators, prey and habitat characteristics that structure food webs and determine species abundance. In order to understand the behavioural responses of prey to the changes in the north-west European landscape, I will focus on the European hare as a case study. In European hares, risk effects are thought to be particularly strong, because they are solitary, free-ranging and do not make use of a burrow for escape (Creel, 2011). Additionally, European hares have developed a very strong anti-predator strategy, as they are built for flight, while at the same time they can be immobile, vigilant and cryptic (Focardi \& Rizzotto, 1999).

\section{THE EUROPEAN HARE}

In 2008, the European Hare (Lepus europaeus Pallas, 1778) has moved up on the IUCN red list from Lower-Risk-Least Concern in 1996 towards Least Concern (Smith \& Johnston, 2008). Although the species is still widespread and abundant from north-western Spain to halfway Mongolia, its population trend (i.e., hare hunting bags) decreased (IUCN, 2017) since the 1960s (Broekhuizen, 1982; Smith et al., 2005) and earlier. Denmark experienced an 85\% decrease in hare hunting bags between 1940 and 2001 (Olesen \& Asferg, 2006), whereas hare numbers hunted in Switzerland decreased about 85\% between 1930 and 2000 (LundströmGilliéron \& Schlaepfer, 2003). A similar pattern was found in Norfolk, where the total number of hares shot showed a decreasing trend from the 1910 s till the 1980s (Hutchings \& Harris, 1996). In 2009, NEM reported a 30\% decrease in European hare population numbers in the Netherlands from 1997 to 2008. Although there is still no consensus as why European hares have declined (Smith et al., 2005), a broad spectrum of possible causes has been put forward (Hutchings \& Harris, 1996; Lamarque et al., 1996; Roedenbeck \& Voser, 2008) that probably interacted in complex ways (Smith et al., 2005). The intensification of agriculture has been suggested to be the 'ultimate cause of hare population declines' (Smith et al., 2005, p.1), resulting in the homogenisation of the landscape (i.e., a loss of habitat diversity, structure and quality). This homogenisation of the landscape may have mediated the impact of predators (Schneider, 2001; Smith et al., 2005), because during the same period hare population numbers were strongly negatively correlated to population numbers of predators (i.e., especially red fox (Vulpes vulpes) (Reynolds \& Tapper, 1995; Schmidt et al., 2004; Knauer et al., 2010).

Various species of predators prey on the European hare (Huber, 2004; Tapper \& Yalden, 2010), mainly belonging to four different types, namely (a) birds of prey 
(e.g., Northern goshawk, Eurasian sparrow hawk, short-eared owl, long-eared owl, little owl, Eurasian buzzard, western marsh harrier, hen harrier, Montagu's harrier, Tawny owl, and barn owl), (b) omnivorous birds (e.g., great egret, grey heron, purple heron, and white stork), (c) carnivorous mammals (e.g., domestic or feral cat, stoat, European mink, least weasel, European polecat, and American mink), and (d) omnivorous mammals (e.g., domestic dog, beech marten, European pine marten, and red fox). In this thesis I will focus on adult hares solely, even though predation risk of hares depends on their life stage. Additionally, I will avoid the breeding season to reduce the complexity of the research.

The space use of adult hares shows a distinctive daily rhythm related to their digestive physiology as a herbivore (Tapper \& Yalden, 2010). During daytime they rest, digest, and re-ingest their pellets, often in cover (Neumann et al., 2011). During the night they feed mainly on herbs and grasses in less covered areas. As a result, hares often commute daily between the two habitat types (Schai-Braun et al., 2012) that may differ in predation risk. The characteristics of the habitat, such as the presence of edge habitat, vegetation structure, cover and the openness of the landscape (Bresinski, 1983; Focardi \& Rizzotto, 1999; Caravaggi et al., 2015; Smith et al., 2005), are assumed to determine the way hares respond to predation risk. For example, in habitat with high cover (e.g., tall vegetation) hares effectively make use of morphological crypsis to reduce flight distance. In habitat with low cover hare make use of flight to escape predators (Focardi \& Rizzotto, 1999). The use of habitats that differ in predation risk and the strong response to predation risk makes the European hare the ideal species to study the effects of habitat characteristics and predation risk and their interactive effects on prey species. This will help us to understand the responses of prey to the changes in the north-west European landscape.

\section{THE GAPS IN KNOWLEDGE}

In this thesis, I focus on five aspects of the European hare that can be affected by predation risk, namely: movement, space use, foraging behaviour, body condition and reproductive output.

Studying movement offers the potential to understand the relative riskiness of a habitat (Valeix et al., 2010) because habitat characteristics affect prey movement responses (Broekhuis et al., 2013) and the rate at which predators succeed in preying upon animals (Gorini et al., 2012). Although movement responses to predation risk have been investigated in correlative field studies (e.g., Valeix et al., 
2009), the interaction between predation risk and habitat characteristics on the movement responses of mammal prey has not as yet been tested experimentally in a field setting.

Space use and foraging behaviour are affected by habitat characteristics, such as food quality and quantity (Barboza et al., 2009), the risk of predation (Laundré et al., 2001), and the behaviour of fellow prey (i.e., prey that shares a common predator and competes for enemy free-space, but that can also compete over food) (Holt \& Lawton 1994). Although the effects of food quality and food quantity and predation risk received some attention to date, the relative importance of top-down and bottom-up factors (Dobson, 2014) for the space use and foraging behaviour of prey is not well understood in the presence of competitors (Morris, 2009; DeCesare et al., 2010). By investigating the relative importance of these factors that affect behavioural trade-offs in complex landscapes, we can get insight into the mechanisms that determine space use and foraging behaviour of prey species.

Prey body condition and reproduction can be negatively affected by anti-predator behaviour, because of the energetic or physiological costs involved (Preisser et al., 2005; Creel \& Christianson, 2008; Sheriff et al., 2009). However, effects of chronic exposure to risk of multiple predators to the reproductive output of prey species are poorly investigated. By studying the risk effects of multiple predators on prey fitness, I hope to signify predation-risk effects (Zanette et al., 2014).

\section{THESIS OBJECTIVES}

The objective of the thesis was to investigate the effects of predation risk and habitat characteristics on European hare. This will help us to understand the responses of prey to the changes in the north-west European landscape.

In this thesis I report on:

1. The effect of elevated predation risk on the movement of the European hare in patches of different vegetation structure

2. The effect of predators, vegetation structure and competitors on the space use of the European hare

3. The effect of predators on the foraging behaviour of the European hare, relative to the effect of food quality, food quantity and competitors

4. The effect of chronic exposure to predation risk of multiple predators on the body condition and reproductive output of the European hare 


\section{THESIS OUTLINE}

The conceptual outline of the thesis is shown in figure 1.1. In chapter 2 I report on the effects of predation risk by predators and vegetation structure on the movement response (i.e., space use) of European hare. In chapter 3 I report on the effects of predation risk by predators, vegetation structure and competitors. In chapter 4 I report on the effects of predation risk by predators, competitors, vegetation structure and forage quality and quantity. Whereas, in chapter 5, I report on the effects of predation risk by multiple predators on the body condition and reproductive output of the European hare.

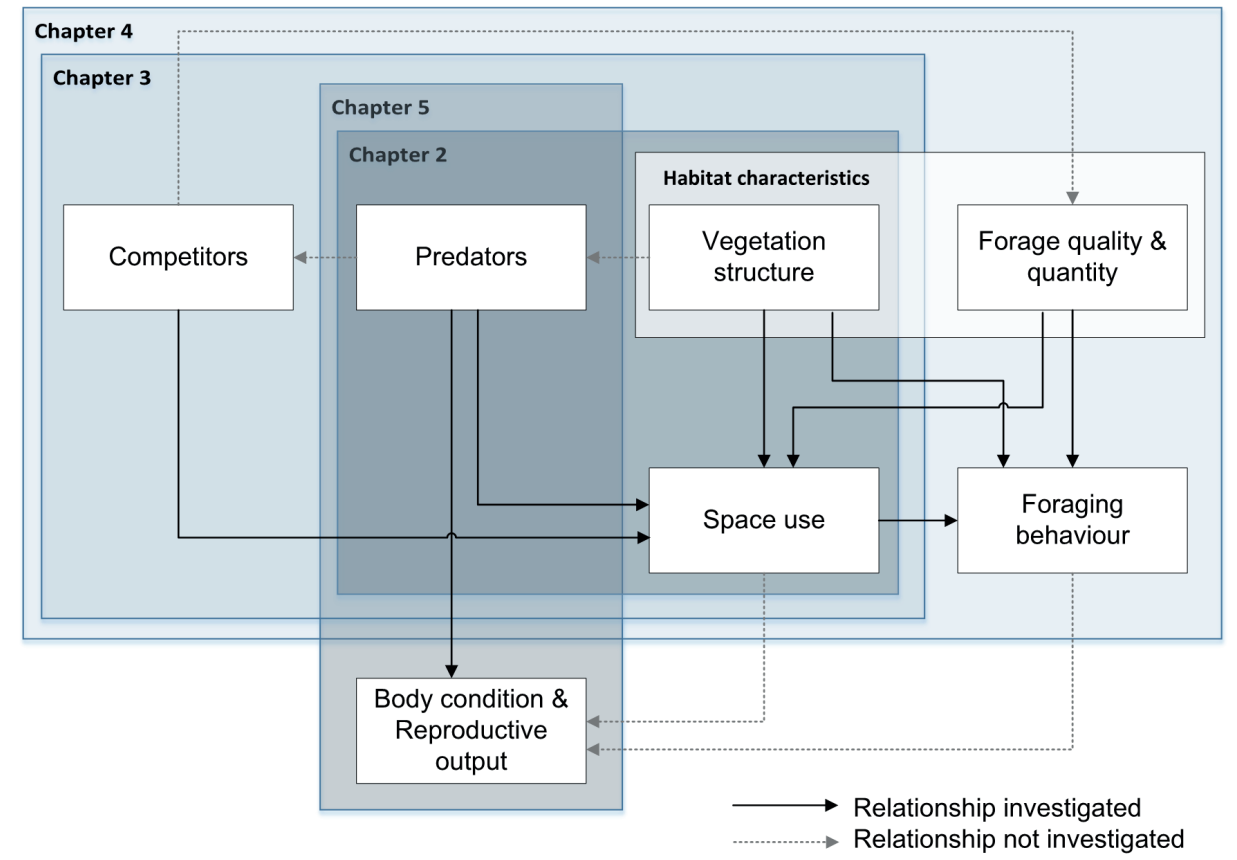

Figure 1.1: Conceptual outline of thesis chapters.

Arrows indicate relationships between factors.

Reactive movement responses of prey are affected by habitat characteristics, such as cover, which determine predation risk (Focardi \& Rizzotto, 1999; Smith et al., 2005). I expected that open habitats with low cover facilitate predator detection, movement and escape, while closed habitats reduce the ability to detect predators and hinder movement. In chapter 2 I tested this hypothesis by repeatedly subjecting hares to the non-lethal predation risk of a leashed dog in a cross-over design on the predator-free island of Schiermonnikoog. I then measured the reactive 
movement responses of GPS-collared hares in patches with different vegetation structure on a short (i.e., several hours) and long (i.e., 24 hours) timescale.

Habitat characteristics, and the presence and behaviour of the predator (Sih, 2005) and fellow prey (Holt, 2009) determine the risk experienced by prey. This results in 'risky places' and 'risky times' that affect the space use of prey (Creel et al., 2008, i.e., landscape of fear, Laundré et al., 2001). However, the influence of habitat characteristics on the threat level of a predator and fellow prey or the interactions between these factors on prey space use was not well understood. Therefore, in chapter 3, I tested the effects of predators, fellow prey and habitat characteristics on the space use of two sympatric prey species. I studied a onepredator, two-prey system in a coastal-dune landscape with the European hare and European rabbit (Oryctolagus cuniculus) as fellow prey and red fox (Vulpes vulpes) as their main predator. Camera traps were used to observe space use of each species that was quantified as an index of the residence time.

Prey space use and foraging behaviour is affected by a trade-off between food quality, food quantity, predation risk and the effects of competitors. Prey is expected to forage in low-risk patches of low-quality food when predators and competitors are more active (Hernández \& Laundré, 2005). Foraging in patches of low-quality food will cost more time, while foraging efficiency is reduced. Small to medium-sized prey are expected to be affected more strongly by predation and competition, than by food quality and quantity (Sinclair et al., 2003; Brown \& Kotler, 2004; Hopcraft et al., 2010). In chapter 4, I tested the relative importance of predator and competitor activity and forage quality and quantity for the proportion of time spent in a vegetation type, and the proportion of time spent foraging by the intermediate-sized herbivore European hare. I equipped twelve hares with GPS and accelerometers in a coastal-dune landscape to investigate space use and foraging behaviour of medium-sized prey. Hand-plucked samples of important plant species in the diet of hare were used to analyse forage quality and quantity, whereas the activity of predators and competitors was investigated using camera traps.

Body condition and reproductive output of prey are supposed to be negatively affected by chronic predation risk (Hawlena \& Schmitz, 2010; Zanette et al., 2014). In chapter 5, I therefore tested the correlation between chronic exposure to predation risk of multiple predators and body condition and reproductive output of European hare from 13 hunting leases in The Netherlands. Density of all predators year-round present was estimated. I expressed the chronic exposure to predation risk as the sum of the field metabolic rates of all predator species. To describe body condition, I extracted four components of the body measurements 
of shot hares by a principal component analysis. Reproductive output of hares was estimated as the number of placental scars.

Finally, in chapter 6, I present the synthesis of the results and the discussions, and how this contributes to the understanding of the relative importance and role of predation risk for prey. 




\section{CHAPTER 2}

\section{Strong reactive movement response of the medium-sized European hare to elevated predation risk in short vegetation}

Weterings, M.J.A., Zaccaroni, M., Van der Koore, N., Zijlstra, L.M., Kuipers, H.J., Van Langevelde, F., Van Wieren, S.E.

Published in Animal Behaviour (2016) 115: 107-114

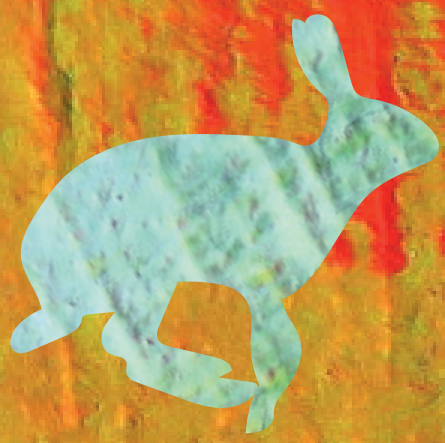




\section{ABSTRACT}

Reactive movement responses of prey are affected by habitat characteristics, such as cover, which determine predation risk. Open habitats with low cover facilitate predator detection, movement and escape, while closed habitats reduce the ability to detect predators and hinder movement. We performed a field experiment using nonlethal predators to study the reactive movement responses of medium-sized prey in patches with different vegetation characteristics related to elevated predation risk. Ten GPS-collared, free-ranging European hares, Lepus europaeus, were repeatedly subjected to a leashed dog and two humans in an experimental crossover design. Linear mixed models were used to assess the effect of the treatment and its interaction with vegetation parameters on the movement behaviour of the European hare. The reactive movement response was best explained by the model that included the interaction between elevated predation risk and vegetation structure. A strong immediate response was found in short vegetation up to $1 \mathrm{~h}$ after the treatment ended. The effect extended beyond the duration of the treatment and was synchronized with the resting and foraging period over the next $24 \mathrm{~h}$. The distance covered between resting and foraging grounds was negatively affected, while use of less risky, low quality vegetation during resting and foraging was favoured. Medium-sized prey species exhibit strong behavioural responses to the perceived predation risk, which we demonstrate here for the European hare. An elevated predation risk, for example by dogs, can trigger costly behavioural responses in these medium-sized prey species. 


\section{INTRODUCTION}

Predators affect prey by increasing mortality and altering behaviour (Creel \& Christianson, 2008; Lima \& Dill, 1990). The impact of predators scaring prey (risk effects) is at least as strong as or stronger than predator density effects (Preisser et al., 2005). These risk effects induce antipredator behaviour, such as predator avoidance, and negatively affect foraging efficiency (Møller, 2008).

Prey can show both predictive and reactive responses to perceived predation risk. Predictive responses are based on previous knowledge and are thus not immediate, but are expressed by long-term habitat selection (Valeix et al., 2009). Reactive responses are based on current knowledge resulting in immediate responses during an encounter with a predator. Habitat characteristics are expected to affect reactive movement responses (Broekhuis et al., 2013; Ydenberg \& Dill, 1986) and the rate at which predators succeed in preying upon animals (Gorini et al., 2012). Previous studies have characterized the riskiness of habitats (i.e. amount of cover) by vegetation density, height or structure (Bissett \& Bernard, 2007; Riginos \& Grace, 2008). In general, open, less structured, relatively homogeneous habitats with little cover lead to high visibility for both prey and predators. This type of open habitat reduces the opportunity for hiding and concealment, but will cause prey to be more easily alerted (Focardi \& Rizzotto, 1999). Little cover facilitates movement or escape. In contrast, closed, structured, relatively heterogeneous habitats provide cover, but they reduce the ability to visually detect predators and hinder movements (Lima, 1992).

Studying movement offers the potential to understand the relative riskiness of habitats (Valeix et al., 2010). Movement allows animals to optimally use their habitat by trading off energy investment and expenditure (Johnson et al., 1992). Overall, faster movement can be expected in open habitats when predation risk is elevated than in closed habitats (Hauzy et al., 2010). Although reactive responses have been the focus of various correlative field studies (Valeix et al., 2009) and experimental laboratory studies (mostly aquatic, Waggett \& Buskey, 2007), the interaction between elevated predation risk and cover on reactive movement responses of mammal prey has not as yet been tested experimentally in a field setting.

Predation effects are dependent on body mass. Small (e.g. rodent) and medium (e.g. lagomorph) prey species are expected to be top-down regulated by predation, whereas population sizes of large prey species (e.g. bovines) will be determined 
by food availability (Hopcraft et al., 2010; Sinclair et al., 2003). For medium-sized prey, elevated predator encounters in open habitats are expected to result in a reactive movement response characterized by increased speed, or longer stretches (Waggett \& Buskey, 2007) alternating with small angle directional changes to allow prey to escape predators. In closed habitats, prey are expected to remain motionless due to the presence of cover.

The European hare, Lepus europaeus, is a medium-sized, solitary, noncentralplace herbivore found in open areas. Most medium sized mammals like the European hare are nocturnal or crepuscular and share visual and auditory adaptations for living in low light conditions (Ashby, 1972); thus they presumably perceive predation risk in a similar way. Hare populations have decreased consistently and substantially in Europe since the 1960s (Tapper \& Parsons, 1984). A broad spectrum of possible causes has been postulated (Smith et al., 2005), including recent increases in their main predator, the red fox, Vulpes vulpes (Knauer et al., 2010). Smith et al. (2005) identified agricultural intensification as the ultimate cause of their decline, leading to more homogeneous landscapes that are lacking in cover. As a consequence, hare populations could experience greater predator impact (Schneider, 2001). Risk effects are especially profound in hares, as they cannot rely on aggregation or a burrow to reduce predation risk (Creel, 2011). Hares have developed a very strong active antipredator strategy; they are built for flight, while at the same time they can be immobile, vigilant and cryptic.

We hypothesized that the reactive movement response of medium-sized prey in low cover habitats would increase in speed with an elevated predation risk compared with movement in high cover habitats. To test this hypothesis we performed a field experiment and manipulated predation risk using nonlethal predators. European hares were used as the medium-sized prey species and were affixed with GPS collars to more accurately track movement.

\section{METHODS}

\section{Study area}

The study was conducted on the island of Schiermonnikoog $\left(53^{\circ} 30^{\prime} \mathrm{N}, 6^{\circ} 10^{\prime} \mathrm{E}\right)$, The Netherlands. Two 200 ha experimental areas were selected, both at least $1 \mathrm{~km}$ apart to prevent overlap of hare territories between the two sites (figure 2.1). The sites had similar vegetation types and structures, containing successional stages from pioneer to climax stage, with a patchy distribution of both homogeneous 
and heterogeneous dune and salt marsh habitat. The salt marsh was a lowland area under tidal influence positioned parallel to the coastline. Currently, a fluctuating population of between 300 and 600 sedentary European hares live on the island (Van Wieren et al., 2006), with an average home range (values are given as mean $\pm \mathrm{SD}$ throughout the manuscript) of $27.3 \pm 9.0$ ha (Kunst et al., 2001).

On the island of Schiermonnikoog, birds of prey such as the marsh harrier, Circus aeruginosus, goshawk, Accipiter gentilis, and common buzzard, Buteo, and feral cats prey upon hares (Van Wieren et al., 2006), albeit mainly on leverets and juveniles during the reproductive season. Thus, adults experience low natural predation risk. Moreover, hunting has not been allowed since 1996.

\section{Experimental design}

European hare body weights increase from midway through the autumn to overcome the reduction in resource quality and quantity in winter and to build up fat reserves. These reserves are necessary for reproduction; hares are capital breeders, at least during their first litter (Valencak et al., 2009). It can be assumed that, owing to their accumulated body fat, European hares respond maximally to predation risk in autumn and the onset of winter (Luttbeg et al., 2003), making antipredator behaviour more pronounced. The study was therefore conducted outside the reproductive season, from 17 November to 22 December 2012.

To test reactive movement responses to elevated predation risk, an experimental cross-over design with three nonlethal predators (i.e. a dog and two humans) was performed. Because both predators and disturbers can cause similar antipredator behaviour (Frid \& Dill, 2002), such behaviours are thought to be a generalized response to stimuli that indicate potential predation risk (Koops, 2004). Sheriff et al., (2009) demonstrated that nonlethal dog exposure had a negative effect on the reproductive output of snowshoe hares. It was thus likely that exposure to a nonlethal dog in this study would trigger antipredator behaviour of our prey species. Reactive movement responses of European hares were measured using GPS data loggers that stored one position fix every 6 min for 2 x 12 days. Previous work demonstrated that patterns of activity and movement of brushtail possums, Trichosurus vulpecula, were affected by tagging and could persist for at least 4 days (Dennis \& Shah, 2012). Therefore, the initial experiment was preceded by a 5-day settling down period (Petrovan et al., 2013) and subsequent experiments were separated by a 5 -day washout period, during which the hares did not receive any treatment. We assumed that our treatment did not impact the hares more strongly than the tagging itself. Thus, we assumed that a 5-day washout period 
eliminated previous treatment effects and minimized carry-over effects (Ruxton \& Colegrave, 2011).

In each of the two experimental areas, five healthy European hares (three females and two males) with an average bodyweight of $3291 \pm 237 \mathrm{~g}(\mathrm{~N}=10)$ were caught and equipped with a GPS transmitter positioned on a neck belt. We used lightweight GPS transmitters ( $69 \mathrm{~g}, 2.11 \pm 0.15 \%$ of body weight) with a radio link for wireless communication (Type A, E-obs GmBH, Gruenwald, Germany) to minimize disturbance of the experimental subjects. Body weight was measured, as it can influence movement patterns and habitat use (Prevedello et al., 2010). To capture study animals, hares were flushed by a line of beaters and caught using eight Speedset static hare nets (ca. $90 \mathrm{~m} \mathrm{x} 45 \mathrm{~cm}$, with $13 \mathrm{~cm}$ full mesh; JB's Nets, Alexandria, U.K.) positioned in a T shape. Hares that were captured in the nets were removed within 2-3min by trained experts standing under cover in front of the nets. After capture, the hares were temporarily kept in darkened wooden boxes ( $20 \times 30 \mathrm{~cm}$ and $25 \mathrm{~cm}$ high) with ventilation holes to reduce excess body heat and were blindfolded by a hood to reduce visual stimulation and stress (Paci et al., 2012). Tagging proceeded immediately after all hares in the area were flushed. The hooded hares were gently stabilized by two persons and tagged without sedation (Gerritsmann et al., 2012); handling was kept to a minimum and took $4 \mathrm{~min}$ or less on average. Experimental design, capturing and tagging of hares were approved by the Wageningen University Animal Experiment Committee (no. 2012083) and followed the EU Directive 2010/63 on the protection of animals used for scientific purposes. 


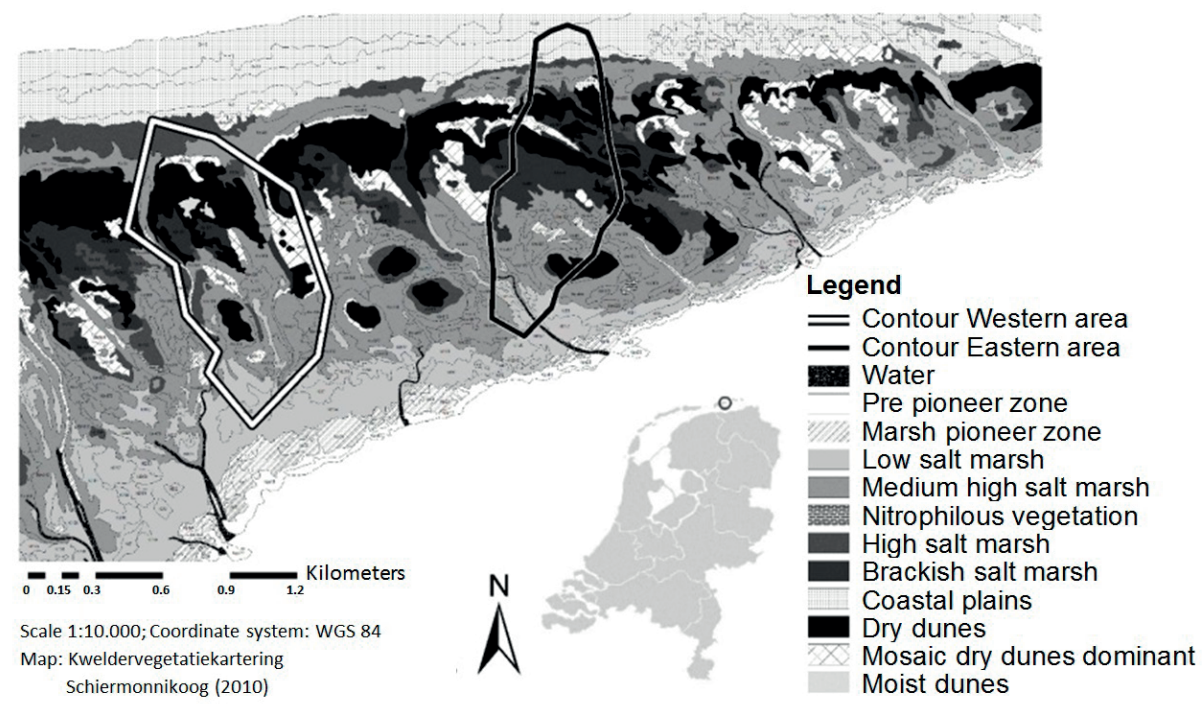

Figure 2.1: Delineation of the study area on Schiermonnikoog, including the two experimental areas in the salt marsh; the western (left) and eastern (right) areas are shown with white and black contour lines, respectively.

During the experimental treatment, two persons walked with a nonbarking and leashed ( $\pm 5 \mathrm{~m}$ long line) 4-year-old $\operatorname{dog}$ (black Labrador retriever) through the centre of hare activity for $57 \pm 11 \mathrm{~min}$. The centre of hare activity (determined by the locations of all individuals) was updated daily using new remotely recorded GPS locations obtained from the hares' transmitters; locations were determined using a BaseStation (b5, E-obs GmBH, Gruenwald, Germany). During the first 12 days, the treatment was executed in the western area every day and the eastern area served as the control area. After the 5-day washout period, the treatment was executed in the eastern area for 12 consecutive days, while the western area served as the control area. Hares did not move between study areas because the minimum distance that separated the GPS locations of the collared hares in the two study areas was $537 \mathrm{~m}$. The onset of the experiment was on average $72 \pm 35 \mathrm{~min}$ before sunset (i.e. during peak hare movement (Schai-Braun et al., 2012)), which began at 1623 hours (GMT+1). At that time of the day hares become active (Schai-Braun et al., 2012). This timing allowed us to measure potential reductions as well as increases in movement activity as a response to the treatment.

\section{Data preparation}

Accuracy of all GPS collars was measured in the habitats studied prior to animal tagging. After data collection, we used only those GPS (E-obs GmbH GPS log- 
gers, Ublox LEA-4T) locations with a horizontal inaccuracy of less than $10 \mathrm{~m}$, improving the GPS accuracy from $8.63 \pm 9.62 \mathrm{~m}$ to $5.82 \pm 4.06 \mathrm{~m}$. Spatial data were corrected for location errors (Bjørneraas et al., 2010; Friar et al., 2004), which reduced the original data set to $66.5 \%$ of its original size. First, GPS data were averaged over two subsequent GPS locations around a single gap when their Euclidean distance was smaller than $18 \mathrm{~m}$ (mean GPS accuracy $\pm 3 \mathrm{SD}$ ). Subsequently, we removed 'spikes' where the angle between three successive GPS locations was greater than $150^{\circ}$ and smaller than $210^{\circ}$. Lastly, we removed cases where calculated speed over ground was greater than $15 \mathrm{~m} / \mathrm{s}$ or the distance travelled between the 6 min intervals was greater than $1000 \mathrm{~m}$.

We parameterized the riskiness of habitats in classes based on the dominant vertical vegetation structure (VST), primarily using vegetation height $(0 \mathrm{e} 1 \mathrm{~m})$. Low cover represented high-risk vegetation with a short height, while high cover implied the opposite. Habitats were placed on an ordinal scale from low cover to high cover as follows: bare soil (BS, $0 \mathrm{~cm})$; short herb/grass layer $(\mathrm{SO}, 0-30 \mathrm{~cm})$; tall herb/grass layer (TA, 30-100 cm); shrubs $(\mathrm{SH}, 0-50 \mathrm{~cm}$ ). Geographical analysis (ArcGIS 10.1) was used to obtain the vegetation structure from landscape maps (1:10 000 with an accuracy of $2 \mathrm{~m}$, based on false-colour aerial photography, average patch size 0.66 ha; Pranger \& Tolman, 2012). We also included two additional vegetation parameters: the Euclidean distance (DIS) between European hare GPS location and the nearest vegetation patch edge, and patch edge length (EDG). Not only do structure-rich landscapes have a high edge to area ratio (Gorini et al., 2012), European hares prefer edge habitat, and their body weight (condition) is related to edge length (Wincentz-Jensen, 2009).

\section{Data analysis}

We ran multiple linear mixed models (LMM) to assess the effects elevated predation risk, and its interaction with all three vegetation parameters, on the movement behaviour of European hare. We quantified the movement behaviour by calculating the stretch lengths of the movement paths (i.e. the distance between two fixed points), which indicates the net displacement distance covered by the hares during the 6 min interval between the GPS fixes. Predictor variables were grouped into three categories: (1) treatment (and its interaction with the $1 \mathrm{~h}$ time span before, during and after the treatment); (2) vegetation parameters; and (3) control variables. Vegetation parameters consisted of VST, DIS and EDG. We included the two plausible control variables most relevant for hare movement, namely the time to sunset (Schai-Braun et al., 2012) and weight (Prevedello et al., 2010). We generated 14 candidate models from the combinations of these cat- 
egories, including an intercept- and treatment-only model for comparison (table 2.1). Small sample Akaike information criterion (AICc) values were used to select the best fitting 95\% weighted models. Candidate models were used to assess the relative strength of our hypothesis, following Grueber et al. (2011). To prevent over prediction, the maximum number of predictor variables in any model never exceeded five, following Burnham and Anderson (2002).

Individual hares on separate days were used as subjects in the LMM to overcome autocorrelation in the covariance matrix between consecutive days. The GPS interval was set as the repeated measure. The autoregressive moving average (ARMA $(1,1))$ covariance structure was selected using restricted maximum likelihood (REML) estimation based on minimum AICc values. Maximum likelihood (ML) was used to compare the different models.

\section{RESULTS}

We found that the reactive movement response of European hares was best explained by the model including the interaction between the treatment and vegetation structure, with the time until sunset and body weight as controlling variables (table 2.1; see Appendix 2, table A2.1 for beta coefficients and standard errors of the best fitting model).

Models that included VST had a better fit than models that included the DIS, while EDG performed worst of the vegetation parameters. Models that included an interaction between the treatment and the vegetation structure outperformed similar models containing only the main effects of the treatment and vegetation structure. In contrast, models including the main effects of DIS and EDG had a better fit than models that included the interaction of these parameters with the treatment. There was no significant difference in movement response between the two study areas $(\mathrm{F} 1,442=1.17, \mathrm{P}=0.28)$. Day, as a random factor, was not significant (Wald $=0.51, \mathrm{P}=0.61$ ), suggesting that habituation to the treatment was not relevant. 
Table 2.1: Results of the linear mixed model selection, the effect of elevated predation risk and its interaction with vegetation parameters on the movement response of European hares

\begin{tabular}{|c|c|c|c|c|c|c|}
\hline Model type & Rank & Model & $K$ & $\mathrm{AICc}$ & $\triangle \mathrm{AICc}$ & $w_{i}$ \\
\hline Intercept & 14 & Intercept & 4 & 8995.0 & 294.0 & $<0.01$ \\
\hline \multirow[t]{2}{*}{$\mathrm{T}$} & 10 & $\left(\mathrm{~T}^{*} \mathrm{HR}\right)$ & 9 & 8782.2 & 81.2 & $<0.01$ \\
\hline & 8 & $\left(\mathrm{~T}^{*} \mathrm{HR}\right)+\mathrm{VST}$ & 12 & 8767.7 & 66.7 & $<0.01$ \\
\hline \multirow[t]{3}{*}{$\mathrm{T}+$ vegetation parameter } & 9 & $\left(\mathrm{~T}^{*} \mathrm{HR}\right)+\mathrm{DIS}$ & 10 & 8779.7 & 78.7 & $<0.01$ \\
\hline & 11 & $\left(T^{*} H R\right)+E D G$ & 10 & 8783.2 & 82.3 & $<0.01$ \\
\hline & 2 & $\left(T^{*} \mathrm{HR}\right)+\mathrm{VST}+\mathrm{TS}+\mathrm{W}$ & 14 & 8710.1 & 9.1 & 0.01 \\
\hline \multirow[t]{3}{*}{$\mathrm{T}+$ vegetation parameter + control } & 3 & $\left(\mathrm{~T}^{*} \mathrm{HR}\right)+\mathrm{DIS}+\mathrm{TS}+\mathrm{W}$ & 11 & 8716.9 & 15.9 & $<0.01$ \\
\hline & 5 & $\left(\mathrm{~T}^{*} \mathrm{HR}\right)+\mathrm{EDG}+\mathrm{TS}+\mathrm{W}$ & 12 & 8722.6 & 21.6 & $<0.01$ \\
\hline & 7 & $\left(\mathrm{~T}^{*} \mathrm{HR} * \mathrm{VST}\right)$ & 27 & 8760.5 & 59.5 & $<0.01$ \\
\hline \multirow[t]{3}{*}{$\mathrm{T}^{*}$ vegetation parameter } & 12 & $\left(\mathrm{~T}^{*} \mathrm{HR} * \mathrm{DIS}\right)$ & 13 & 8784.2 & 83.2 & $<0.01$ \\
\hline & 13 & $(\mathrm{~T} * \mathrm{HR} * \mathrm{EDG})$ & 13 & 8786.4 & 85.4 & $<0.01$ \\
\hline & 1 & $\left(\mathrm{~T}^{*} \mathrm{HR}^{*} \mathrm{VST}\right)+\mathrm{TS}+\mathrm{W}$ & 29 & 8701.0 & 0.0 & 0.99 \\
\hline \multirow[t]{2}{*}{$\mathrm{T}^{*}$ vegetation parameter + control } & 4 & $\left(\mathrm{~T}^{*} \mathrm{HR} * \mathrm{DIS}\right)+\mathrm{TS}+\mathrm{W}$ & 14 & 8722.5 & 21.5 & $<0.01$ \\
\hline & 6 & $\left(\mathrm{~T} * \mathrm{HR}^{*} \mathrm{EDG}\right)+\mathrm{TS}+\mathrm{W}$ & 15 & 8726.7 & 25.7 & $<0.01$ \\
\hline
\end{tabular}

AICc: Akaike information criterion corrected for small sample size; $\triangle \mathrm{AICc}$ : delta AICc with regard to best fitting model; DIS: distance to nearest edge; EDG: patch edge length; HR: $\mathrm{h}$ block ( $1 \mathrm{~h}$ data segment before, during and after the experiment); K: number of estimated parameters; T: treatment; TS: time to sunset; VST: vegetation structure; W: body weight; wi: Akaike weight or relative weight of each model.

Elevated predation risk increased stretch length compared to control areas in habitats with little structure (i.e. bare soil, low and tall herb/grass layers) during the treatment. This behavioural change was similar $1 \mathrm{~h}$ after treatment, but only in vegetation with low and tall herb/grass layers (figure 2.2). Remarkably, the behavioural response was strongest in shrub vegetation $1 \mathrm{~h}$ before the treatment commenced. Stretch length in the treated areas during the time of treatment was significantly higher in the low herb/grass layers compared with the tall herb/ grass layers. Stretch length in the former was also significantly greater compared with stretch length in the same vegetation structure $1 \mathrm{~h}$ before the treatment took place.

In the control area, the average 6 min stretch length was shortest in shrub vegetation $1 \mathrm{~h}$ before the treatment. Stretch length was also significantly different in shrub vegetation compared with bare soil or vegetation with low and high herb/grass layers during the same time period. Furthermore, in shrub vegetation, 
stretch length differed between the time of treatment and the subsequent $1 \mathrm{~h}$ time period.

The effect of the predation risk treatment on the Euclidean distance between the position of the hares at the start of the dog walk and positions during the rest of the day (figure 2.3) roughly followed the day-night cycle. This cycle could be split in three distinct phases. During the first $2 \mathrm{~h}$ and last $7 \mathrm{~h}$ of the day, the Euclidean distance moved by hares exposed to elevated predation risk was larger compared to hares in the untreated area, but distances were lower for treated hares during the night (figure 2.3). The same pattern was observed for hares in vegetation with tall herb/grass layers (figure $2.4 \mathrm{a}$ ) and in shrub vegetation (figure $2.4 \mathrm{~b})$. Compared with the control group, hares in the treated area were more often present in vegetation with tall herb/grass layers during the night (figure 2.4a), but were less frequently present in this type of vegetation structure during the day. Hares were found in vegetation with tall grass/herb layers in $58.4 \%$ of the total number of fixes. Hares in the treated area were present in shrub vegetation less often during the night, but more often during the day, compared with the control (figure 2.4b). In addition to the increased presence in the shrub layer during the day, treated hares exhibited a larger average stretch length in shrubs $1 \mathrm{~h}$ prior to treatment (figure 2.2). Hares were in shrub vegetation in $15.1 \%$ of the total number of fixes. The difference (treatment - control) in the fraction of time spent by hares on bare soil and in vegetation with short herb/grass layers did not show the distinct pattern seen in the vegetation with tall herb/grass layers and shrub vegetation. 


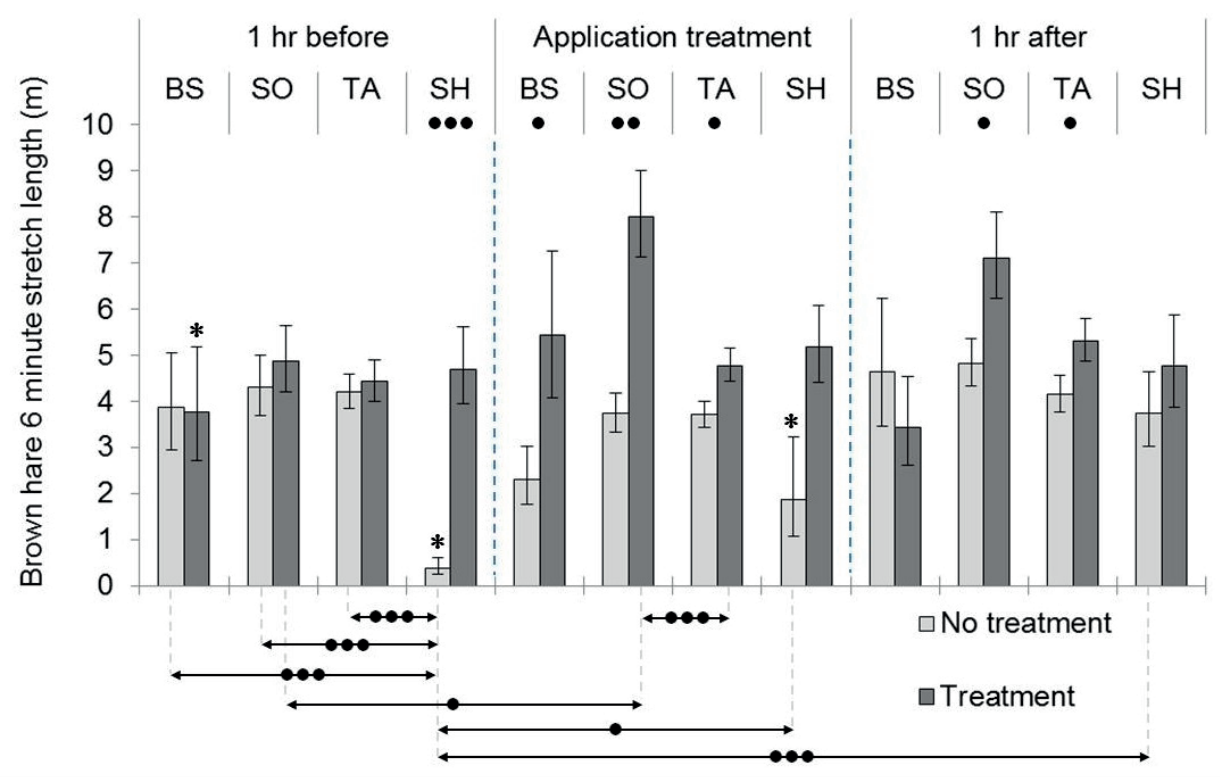

Figure 2.2: Six-minute stretch length of European hare $1 \mathrm{~h}$ prior to, during and after predation risk treatment in different habitats (marginal means $+95 \%$ confidence intervals).

Vegetation structures (\% used) are as follows: BS = bare soil, $0 \mathrm{~cm}(4.4 \%)$; $\mathrm{SO}=$ short herb/grass layer, $0-30 \mathrm{~cm}(26.4 \%)$; $\mathrm{TA}=$ tall herb/grass layer, $30-100 \mathrm{~cm}(62.1 \%)$; $\mathrm{SH}=\operatorname{shrub}, 0-50 \mathrm{~cm}(7.2 \%)$. Dots above the bar graph indicate differences between treated and untreated groups, whereas the dots underneath the columns show differences between types of vegetation structure within a treatment group. $\bullet \mathrm{P}<0.05 ; \bullet \bullet P<0.01 ; \bullet \bullet P<0.001$ (Sidak test). Marginal means were evaluated $0.69 \mathrm{~h}$ before sunset for a European hare with a weight of $3322 \mathrm{~g}$ (average $\mathrm{N}=25.5, *<5$ samples).

\section{DISCUSSION}

We investigated the reactive movement response of European hares following exposure to an elevated predation risk, that is, a leashed dog with two humans, in a mosaic of salt marsh vegetation. Our study is the first to experimentally manipulate and measure the short- and longer-term effects of predation risk on the movement activity of a medium-sized mammal in a field setting (Ferrari et al., 2009).

We showed that there was a strong reactive movement response to elevated predation risk in short vegetation. Vegetation structure was the most important vegetation parameter affecting the reactive movement response of hares, followed by the distance to the nearest vegetation patch and patch edge length. Finally, the effect of the elevated risk treatment extended beyond the immediate time of the 
treatment. Although the effect roughly followed the night and day activity cycle, risk exposure primarily influenced the time spent in vegetation with high cover (shrub), in which prey was found after the sunrise following the treatment.

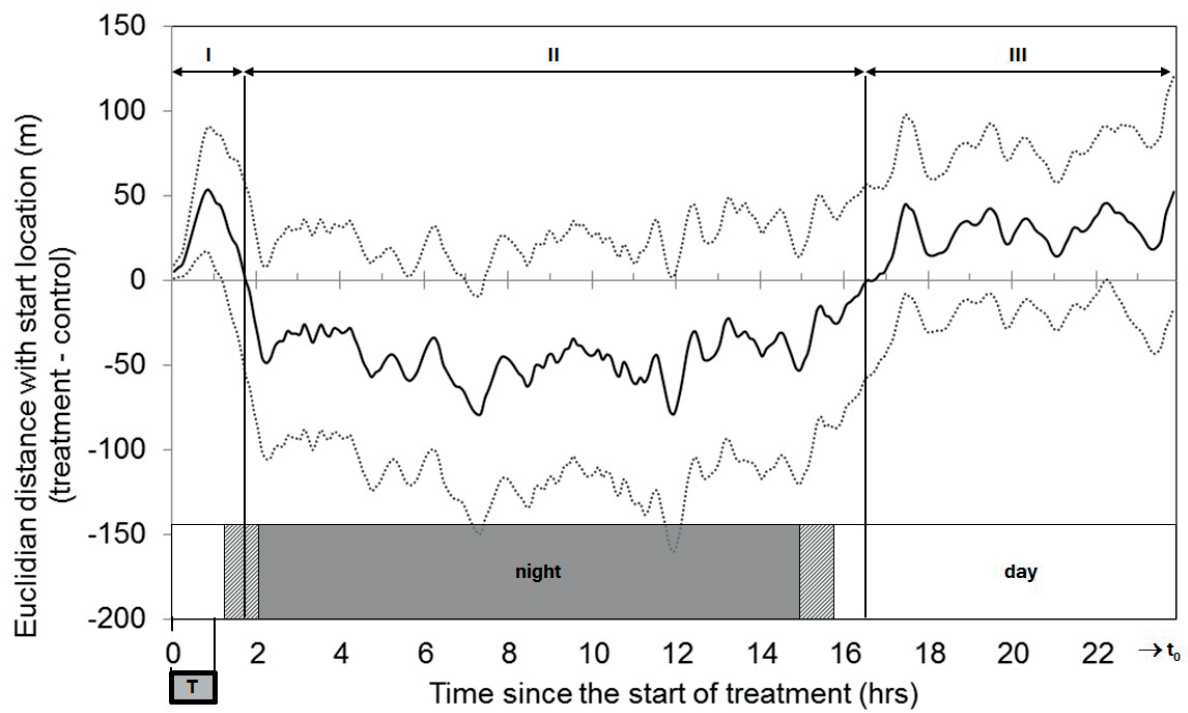

Figure 2.3: Time since the start of the treatment and the Euclidean distance between the start location at $\mathrm{t}_{0}$ and the location of European hares at $\mathrm{t}_{\mathrm{x}}$ (average $\mathrm{N}=50.1$; treatment - control).

Bold black line indicates the mean, the broken lines indicate the upper and lower 95\% confidence intervals. $\mathrm{T}=$ application of treatment. Three phases can be distinguished: I and III, where treatment $>$ control, and II, where treatment $<$ control. The light grey areas indicate dusk and dawn. 

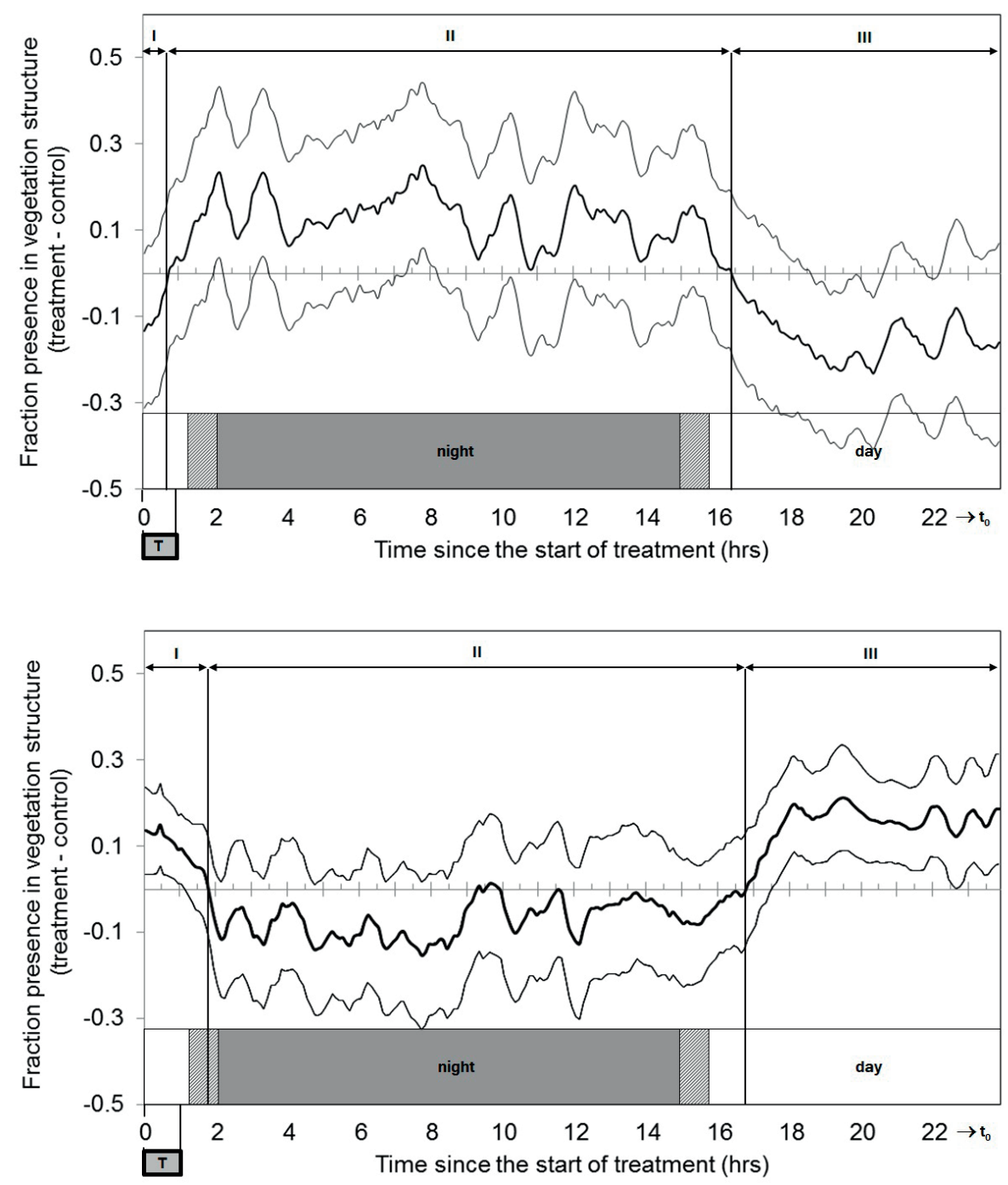

Figure 2.4: The time since the start of the treatment and the fraction of time spent in (a) vegetation with tall herb/grass layer $(30-100 \mathrm{~cm})$ and $(\mathrm{b})$ shrub vegetation $(0-50 \mathrm{~cm})$ by European hares (average $\mathrm{N}=50.1$; treatment - control).

Bold black line indicates the mean, the broken lines indicate the upper and lower 95\% confidence intervals. $\mathrm{T}=$ application of treatment. Three phases can be distinguished: I and III, where treatment $>$ control, and II, where treatment $<$ control. The light grey areas indicate dusk and dawn.

\section{Reactive response}

As hypothesized, the reactive response increased prey average speed only in areas of low cover, while the average speed in high cover did not show a significant 
increase. There are several explanations for these responses. First, low cover is correlated with high visibility and more frequent encounter rates (Gorini et al., 2012). An imminent predation threat can encourage prey to avoid the attention of the predator (Broom \& Ruxton, 2005; Ydenberg \& Dill, 1986) by moving to a safer location with more cover. Second, the spatial distribution of vegetation patches in the salt marsh mosaic may affect habitat-dependent escape tactics (Heithaus et al., 2009). Compared to a more open area, a patchwork of small vegetation patches decreases the detection distance of approaching predators. Prey present in low cover patches seem to increase speed, resulting in movement to new vegetation patches, where 'its escape tactic is most effective' (Lima, 1992, p.598). Third, the interaction between predator type and vegetation structure is important for the perceived predation risk by prey. For example, Hewson (1977) emphasizes the importance of low cover to enable European hares to see approaching predators and thus facilitate flight. High cover benefits stealth-dependent predators (i.e. ambush or stalker), while low cover benefits coursers. During our experiment, prey that recognized the nonlethal predator as a courser could have been avoiding low vegetation to reduce predation risk.

We found an increase in the speed of hares in shrub vegetation over time in the untreated areas around the time of treatment application (i.e. just prior to sunset). At that time of day, European hares increased locomotion to translocate from resting areas to foraging grounds (Schai-Braun et al., 2012). For unknown reasons, the stretch length of hares in shrub vegetation in the treated area $1 \mathrm{~h}$ before the application of the treatment was significantly higher than the control. For the treated prey, the perceived predation risk in shrub vegetation during treatment did not increase the benefits of fleeing to outweigh the benefits of staying (Ydenberg \& Dill, 1986). Vegetation with high cover reduces fear in animals (Stankowich $\&$ Blumstein, 2005). In addition, European hares make use of morphological crypsis in covered vegetation to effectively reduce flight distance (Focardi \& Rizzotto, 1999).

Our results show a clear influence of the daily activity cycle, as seen in the transition from one phase to another, on the effect of treatment over the subsequent $24 \mathrm{~h}$. Although marginally significant, treated prey tended not to move as far as the control group from their original position at dusk during the start of the treatment; nor did they return as close to that position at dawn. Cooper and Sherbrooke (2013) showed that cryptic prey take their own recent movement into account in assessing predation risk, explaining the reduction in speed after an initial flight response. According to models of patch use under predation risk, animals 
either reduce behaviours that attract predators, such as moving, or increase the encounter rate with predators (Brown, 1999). European hares leave their resting areas just before sunset and move to foraging grounds, where they forage during the night (Tapper \& Barnes, 1986). Just prior to sunrise, they relocate to their shelters again. It seems that these transitions from shelter to foraging ground and back are affected several hours after the application of the treatment, resulting in shorter translocations, possibly in an attempt to avoid predator attention. Compared with the control group, prey that experienced an elevated predation risk favoured the high cover vegetation patches during their daily activities over the subsequent $24 \mathrm{~h}$. These patches contained a lower quality of forage, but were less risky (Gorini et al., 2012; Hauzy et al., 2010), and thus indicate an antipredator benefit (Lima \& Dill, 1990).

Continuous disturbance resulting in antipredator behaviour and the effects of behavioural changes on lower trophic levels (i.e. trait-mediated indirect effects) require further attention (Kuijper et al., 2013). Kuijper and Bakker (2005), for example, showed that exclusion of European hares allows the invasion of late successional plant species in some habitats, with shifts in species composition being most pronounced in unproductive areas. Trait-mediated indirect effects of prey may thus have a strong effect in dunes or other unproductive habitats.

Here we used a nonlethal dog as a (cursorial) predator, because we see this experiment as a first step towards evaluating the risk effects on medium-sized prey (Luttbeg et al., 2003). The question remains as to whether these effects were the result of the direct exposure to the humans or the dog, or the result of the dog's odour. Odours can cause habitat shifts, alter activity patterns or reduce non-defensive behaviours, and skin- and fur-derived odours have a stronger longlasting effect on prey species than those from urine or faeces (Apfelbach et al., 2005). However, according to Thaker et al. (2011), ungulates did not avoid areas of high utilization by cursorial predators, probably as olfactory cues associated with coursing predators were not related to predator proximity (Wilkenros et al., 2015; but see Kuijper et al., 2014).

Smaller prey species exhibit stronger behavioural responses to perceived predation risks than larger species (Creel, 2011). Nevertheless, it is not uncommon for prey to compensate behaviourally for losses, for example in foraging, due to risk effects (Luttbeg et al., 2003). Repeated exposure to elevated predation risk, as well as disturbance (Frid \& Dill, 2002), could make the effects on medium sized prey shown here more long lasting, with potential demographic consequences (Sher- 
iff et al., 2011). European hares are sensitive to disturbance, especially by dogs (Hoeksma, 1950) and roads (Roedenbeck \& Voser, 2008). Frequent disturbances could have contributed to the population declines of European hares near human populations (Cromsigt et al., 2013). Young et al. (2011) suggested that dogs should be excluded from critical wildlife habitats, as they and their accompanying humans can affect prey habitat choice and movement activity, and can bring about a costly stress response (Lenth et al., 2008).

\section{Conclusions}

We found an interaction between elevated predation risk and cover on the reactive movement responses of a medium-sized prey, the European hare, in a field experiment. Hares show a strong reactive response to elevated risk in low cover. Vegetation structure most strongly affected the reactive response of hares. We also observed an extended effect of the treatment used in this study, with hares spending more time in high cover vegetation during resting and feeding for at least $24 \mathrm{~h}$ following predator exposure. 


\section{APPENDIX 2}

The coefficients (b) and standard errors (SE) of the best fitting model (the three-way interaction between the treatment, vegetation structure and $\mathrm{h}$ block) were generally higher during the control phase of the experiment than during the treatment phase (table A2.1). Time until sunset was positively related to European hare 6 min stretch length, while a higher European hare body weight was related to shorter stretch lengths.

Table A2.1: Beta coefficients and SEs of the best fitting model [(T * HR * VST) + TS + W] variables related to European hare 6 min stretch length

\begin{tabular}{lccc}
\hline \multicolumn{3}{c}{ HR } \\
\hline Model variable* $^{*}$ & 1 h before & During treatment & $1 \mathrm{~h} \mathrm{after}$ \\
\hline T (control) ${ }^{*}$ VST & & \\
1. $\mathrm{BS}$ & $2.55 \pm 0.62$ & $3.16 \pm 0.79$ & $2.38 \pm 0.81$ \\
2. $\mathrm{LO}$ & $0.077 \pm 0.43$ & $0.67 \pm 0.67$ & $0.076 \pm 0.69$ \\
3. HI & $0.15 \pm 0.46$ & $1.16 \pm 0.69$ & $0.35 \pm 0.71$ \\
4. SH & Ref & $0.88 \pm 0.54$ & $-0.27 \pm 0.58$ \\
T (treatment) ${ }^{\text {VST }}$ & & & \\
1. BS & Ref & $2.10 \pm 0.68$ & $2.11 \pm 0.61$ \\
2. LO & Ref & $0.39 \pm 0.38$ & $-0.20 \pm 0.41$ \\
3. HI & Ref & $0.37 \pm 0.40$ & $-0.070 \pm 0.43$ \\
4. SH & Ref & Ref & Ref \\
\hline
\end{tabular}

HR: h block ( $1 \mathrm{~h}$ data segment before, during and after the experiment); T: treatment; TS: time until sunset (h); VST: vegetation structure [BS, bare soil $(0 \mathrm{~cm})$; LO, low herb/grass layer $(0-30 \mathrm{~cm}) ; \mathrm{HI}$, high herb/grass layer $(30-100 \mathrm{~cm})$; SH, shrub $(0-50 \mathrm{~cm})]$; W: body weight $(\mathrm{g})$; ref: reference category $=$ shrub vegetation $1 \mathrm{~h}$ before treatment.

$*$ Without main effects of three-way interaction. Intercept: $\beta \pm \mathrm{SE}=-2.37 \pm 0.49 ; \mathrm{TS}: \beta \pm \mathrm{SE}=0.34$ $\pm 0.043 ; \mathrm{W}: \beta \pm \mathrm{SE}=-0.0002 \pm 0.0001$. 




\section{CHAPTER 3}

Predators, fellow prey and risk factors: Implications for the space use of two sympatric leporids

Weterings, M.J.A., Ewert, S.P., Peereboom, J.N., Kuipers, H.J., Kuijper,

D.P.J., Prins, H.H.T., Jansen, P.A., Van Langevelde, F., Van Wieren, S.E.

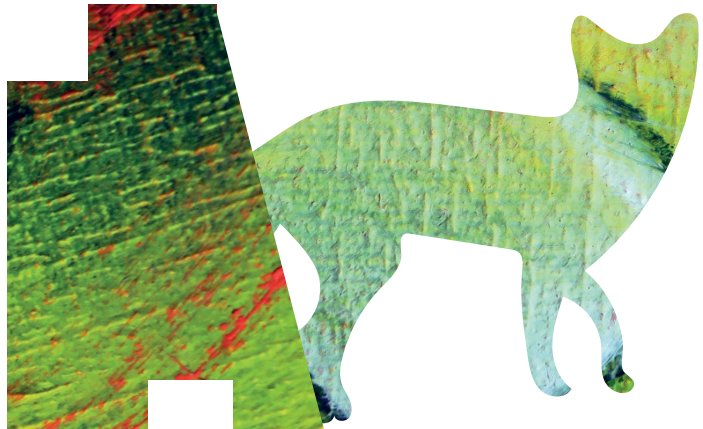




\section{ABSTRACT}

Prey space use is affected by 'risky places' and 'risky times.' Perceived risk depends on the behaviour of the predator and characteristics of the environment. Prey species can also perceive predation risk as a result of the presence and behaviour of fellow prey. However, the influence of environmental characteristics (i.e., risk factors) on the perceived threat level of a predator and fellow prey or the interactions between these factors is not well understood. Our objective was to explore the effects of predators, fellow prey and risk factors on the space use of two sympatric prey species.

We studied a one-predator, two-prey system in a coastal dune landscape in the Netherlands with the European hare (Lepus europaeus) and European rabbit (Oryctolagus cuniculus) as sympatric prey species and red fox (Vulpes vulpes) as their main predator. The space use of each species was observed using camera traps. We quantified the space use as an index of the residence time.

Our prey species differed in their responses to the space use by predators, space use by fellow prey, and risk factors. Activity by rabbits was correlated with activity by predators in time, but uncorrelated in space. Activity by hares was uncorrelated with activity by predators in time, but correlated in space. In the presence of predators, hares that were affected by fellow prey shifted habitat and sought low-risk locations. Predator presence possibly released avoidance and promoted coexistence between prey species in the high-risk habitat, but seem to have no effect in the low-risk habitat. Apparent competition may have reversed the relationship between the space use by predators and risk factors on the space use by prey.

Our study demonstrates that space use of two sympatric prey species is complex. Properties of prey species affect the relations between the space use by predators, space use by fellow prey and risk factors. Additionally, risk factors and species presence interact with the space use by predators or the space use by fellow prey on the space use by prey. 


\section{INTRODUCTION}

Two alternative views dominate studies on the relationship between the use of space by predators and that of their prey (Sih, 2005). If prey avoid locations that contain predators, the space uses of predators and prey are negatively related (Thaker et al., 2011), whereas if predators aggregate in locations with prey, the space uses of predators and prey are positively related (Stephens \& Krebs, 1986). However, prey and predators respond to each other, resulting in a dynamic relationship (Lima, 2002). In this behavioural response race (Sih, 2005), predators select locations and times in response to the use of space by prey (Laundré, 2010), resulting in 'risky places' and 'risky times' (Creel et al., 2008, i.e., landscape of fear, Laundré et al., 2001), whereas prey select locations and times to avoid these risky places and times (Lima \& Dill, 1990).

The use of space by prey is also affected by indirect effects. Indirect effects occur when one species affects another species via a third intermediate species (Holt, 2009). Apparent competition is an indirect effect in which a given prey species experiences more predation risk because of changes in predator abundance (numeric response) or predation rate (functional response) resulting from the presence of fellow prey (Holt, 2009). Short-term apparent competition (aggregative response) occurs if the use of space by a predator is driven by the prey distribution at short time scales (Holt \& Kotler, 1987). Prey species that have a shared predator compete for enemy-free space (Holt \& Lawton, 1994). These species avoid risky places and times that are shaped by spatial and temporal variations in the behaviour of fellow prey.

The perceived risk of a location is a combination of the probabilities of attack and escape (Bednekoff \& Lima, 1998) and is thought to depend on the presence of a predator (Kuijper et al., 2014), characteristics of the environment that determine risk (henceforth risk factors) (Gorini et al., 2012), behaviour of fellow prey (Holt \& Lawton, 1994) and interactions between these factors (Kuijper et al., 2015). The characteristics that determine the probability of an attack are not necessarily the same characteristics that determine the probability of escape (Gorini et al., 2012). The spatial variation in risk factors has a major influence on the relation between the use of space by predators and prey (Chesson, 2000); however, this is not well understood in the presence of fellow prey (Oliver et al., 2009; DeCesare et al., 2010; Wirsing et al., 2010). 
Our objective was to explore the effects of predators, fellow prey and risk factors on space use of two sympatric prey species. We used a one-predator, two-prey system composed of the European hare (Lepus europaeus, Pallas, 1778) and European rabbit (Oryctolagus cuniculus, Linnaeus, 1758) as sympatric prey species and the red fox (Vulpes, Linnaeus, 1758) as their main predator. These prey species are thought to avoid each other (Flux, 2008; but see Stott, 2003).

Rabbits are social central-place herbivores and prefer edge habitat (Barnes \& Tapper, 1986). The presence of rabbits can dilute (Lima \& Dill, 1990) or enhance the risk effects for other prey species, the latter because they are a staple food of several predators (Norbury, 2001), such as the fox. The solitary hare is a non-central-place herbivore that is common in open areas (Barnes \& Tapper, 1986). Foxes can substantially impact hare populations (Schmidt et al., 2004). The effect of hare predation has been suggested to depend on the available vegetation structure, cover and openness of the landscape (Focardi \& Rizzotto, 1999; Smith et al., 2005). Hares are known to use tall vegetation as cover or resting places (Neumann et al., 2011). Additionally, hare space use is sometimes positively (Caravaggi et al., 2015) or negatively (Bresinski, 1983) related to edge habitats, presumably depending on the associated risks of the habitat. The fox is known to make use of linear landscape features, such as edges (Frey \& Conover, 2006) and ecotones (Kiener \& Zaitsev, 2010). Foxes avoid open areas, and depending on prey availability (Halpin \& Bissonette, 1988), select for habitats with protective cover, such as shrub vegetation (Kiener \& Zaitsev, 2010).

Niche overlap between two prey species can drive competition. Although hares and rabbit have a considerable overlap in their diet (Kuijper et al., 2004), it is still unclear what mechanism maintains allopatry or drives competition between the two species (Flux, 2008). The competitive advantage of a species depends on its capacity to reduce the availability of resources for the competitor, but also on its capacity to withstand a reduction in the availability of resources by its competitor (Persson, 1985). Introduced hares have been found to be more successful in replacing rabbits on islands than introduced predators or diseases, however rabbits are equally capable of excluding hares from islands (Flux, 1993). This result suggests that the outcome of the interactions between the two prey species depends on the circumstances, but are asymmetric. Such asymmetric indirect effects are often observed in one-predator, two-prey systems (Chaneton \& Bonsall, 2000). Although we expect asymmetric interactions, the mechanism between hares and rabbits is still speculative. We therefore investigated the following question: how 
does space use by hares and rabbits relate to space use by predators, space use by fellow prey and risk factors, and their interactions?

\section{METHODS}

Field work was conducted in the coastal dune landscape 'Noordhollands Duinreservaat' near Castricum $\left(52^{\circ} 33^{\prime} \mathrm{N}, 4^{\circ} 38^{\prime} \mathrm{E}\right)$ in the Netherlands. The average annual rainfall in the region was $\sim 1000 \mathrm{~mm} /$ year. There were three study areas with small populations of hares and rabbits (Vennewater (VW), Koningsbos (KB) and Infiltration area Castricum (ICAS), figure 3.1). We selected the study sites based on previous sightings of hares and rabbits. The study area contained 13 different vegetation types (Appendix 3, table A3.1) and a mosaic of vegetation, including patches of dune grass, thicket, brushwood and forest. This late succession vegetation situated on fertile soils, enriched by atmospheric nitrogen deposition, has a high biomass productivity (Kooijman et al., 1998), but parts remain open dune grassland vegetation because of grazing by rabbits and cattle. Compared to other areas (Trewhella et al., 1988), the fox density in this coastal dune landscape was estimated to be very high, between five and eight individuals per square kilometre (Mulder, 2005).

\section{Field measurements}

\section{Risk factors}

We investigated open versus half-open vegetation structures, edge versus nonedge locations and shrub height as determinants of the environment that affect perceived risk as mentioned in the introduction. An open vegetation structure provides quality foraging ground for hares and rabbits (Kuijper \& Bakker, 2008), whereas a half-open vegetation structure provides cover for these species and is also used as a resting place by hares (Neumann et al., 2011). 


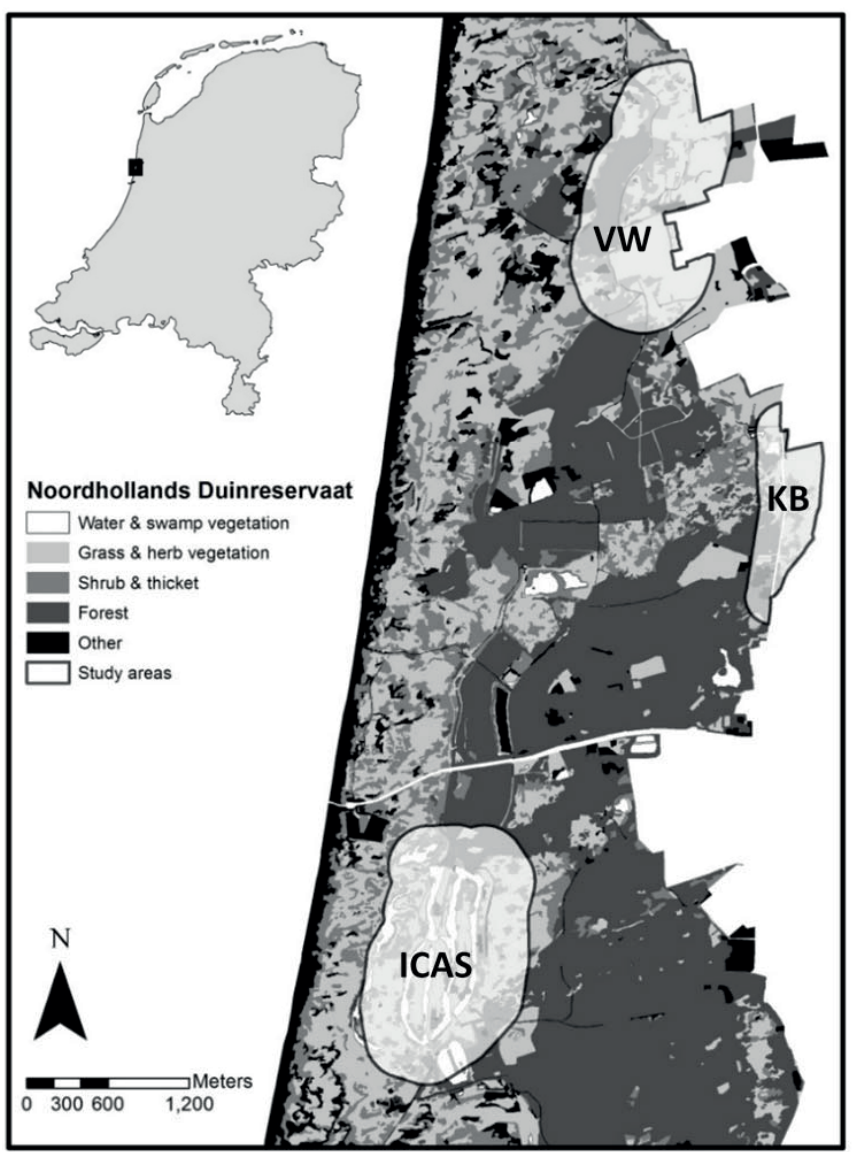

Figure 3.1: Location of the three study areas with fox, hare and rabbit populations: Vennewater (VW), Koningsbos (KB) \& Infiltration area Castricum (ICAS).

\section{Space use and perceived risk}

We distinguished eight vegetation strata, defined by the risk factors. First, we made a distinction between homogeneous patches of open $(>90 \%$ cover of vegetation with an average herb height $<30 \mathrm{~cm})$ and half-open $(\geq 10 \%$ cover of vegetation with an average herb height $\geq 30 \mathrm{~cm}$ ) vegetation structures. Second, the patches were categorized as edge or non-edge locations. Third, each patch near an edge location was subdivided into three different types depending on the surrounding vegetation (forest, thicket, half-open or open) reflecting the differences in perceived risk. An edge habitat was defined as a $12.5 \mathrm{~m}$ strip parallel and adjacent to the edge between two different homogeneous patches of a vertical vegetation structure. We chose these dimensions because the camera traps are able to detect space use of our species at a maximum distance of $12.5 \mathrm{~m}$. 
During five sessions of approximately 15 days each between October 16, 2014, and January 8, 2015, forty-two cameras (Reconyx Hyperfire: HC500 \& HC600, infrared trigger) were randomly placed within the eight strata (210 random points, 3038 successful trap days). The research period was chosen to fall outside of the reproduction period for both prey species to eliminate factors that could cofound space use. Before placement, we took a random sample of possible locations of camera traps from a high resolution (1:5.000) GIS map from Everts et al. (2008; 2009). The locations of the camera traps were interspaced $>50 \mathrm{~m}(>92 \%),>$ $25 \mathrm{~m}$ from waterbodies and $>16 \mathrm{~m}$ from recreational paths. During placement, we positioned cameras at edge locations on the edge itself, facing perpendicularly away from the edge. Cameras at non-edge locations were directed north to avoid overexposure by sunlight during the day. Cameras were mounted on a wooden pole, and the line of sight measured from the camera-lens was calibrated to run parallel to the soil surface at a height of $30 \mathrm{~cm}$ up to at least $5 \mathrm{~m}$ (cf Jansen et al., 2014), without clearing any vegetation. In front of each camera, we measured the shrub height in five locations in a $12.5 \times 12.5 \mathrm{~m}$ orthogonal layout, i.e., related to the maximum detection distance. Cameras were set to record a burst of ten photos (1.s-1) when triggered, without any time lapse between bursts.

We quantified the space use by hares, rabbits and foxes as an index of residence time $(\mathrm{T})$. Residence time of a visit was assessed visually from sequences of camera trap photos. Visits were assumed to be independent if the quiet period in the beginning was longer than 120 seconds. We distinguished two types of residence time per location: 1) average residence time per visit and 2) total residence time. Residence time was corrected for the effective detection area, total deployment time of each camera and stratum (equations 1 and 2).

[Equation 1] Average residence time $\left(\mathrm{s} \cdot \mathrm{h}^{-1} \cdot \mathrm{m}^{-2}\right)$ :

$$
\begin{aligned}
T_{a} & =\frac{t_{\text {avg }}}{\left(r_{\text {eff }}\right)^{2}\left(\frac{\theta_{\text {eff }}}{2}\right) t_{\text {dep }} v_{s}} \\
T_{t} & =\frac{t_{\text {total }}}{\left(r_{\text {eff }}\right)^{2}\left(\frac{\theta_{\text {eff }}}{2}\right) t_{\text {dep }} v_{s}}
\end{aligned}
$$

[Equation 2] Total residence time $\left(\mathrm{s} \cdot \mathrm{h}^{-1} \cdot \mathrm{m}^{-2}\right)$ :

$\mathrm{t}_{\text {avg }}=$ average time spent active in front of a camera per visit (s),

$\mathrm{t}_{\text {total }}=$ total time spent active in front of a camera (s),

$\mathrm{r}_{\text {eff }}=$ effective detection distance in open or half-open stratum (m),

$\theta_{\text {eff }} \quad=$ effective detection angle in open or half-open stratum (rad),

$\mathrm{t}_{\mathrm{dep}}=$ deployment time of the camera $(\mathrm{h})$,

$\mathrm{v}_{\mathrm{s}} \quad=$ average geometric speed of animals in each stratum, relative to the average geometric speed in open or half-open stratum. 
For each species, we measured the detection distance and detection angle of the first capture just before relocating each camera using a wild game viewer. For each species, the effective detection distance and angle were estimated in open and half-open vegetation structures using $\mathrm{R}$ and functions for fitting standard linear covariate detection models to the position of the first capture by camera traps (Rowcliffe et al., 2011; Appendix 3, table A3.2). The effective detection distance and angle defined the area of the habitat that was surveyed. We used the average geometric speed of each species in each stratum relative to the average geometric speed in open or half-open strata to correct for the differences in the effective detection areas. The distance covered by individuals was measured by a tape measure in front of the cameras (Rowcliffe et al., 2011) and was used to calculate the average geometric speed of the species in all strata.

\section{Effects of predator space use and risk factors}

To assess the effects of predator space use and risk factors on prey space use, we tested the effects of 1) the total residence time of the predator, 2) risk factors, and 3) their interactions on the prey average residence time. We assumed that hares and rabbits perceived a high risk when fox total residence time increased. High risk was also perceived in edge habitats, half-open vegetation structures and areas with tall shrubs. Moreover, we assumed that risk associated with fox total residence time, depended on the characteristics of the environment.

\section{Effects of fellow prey space use}

To assess whether apparent competition played a role, we tested the effects of 1) the total residence time of fellow prey, 2) the interaction between the total residence time of fellow prey and predators, and 3) the interaction between the total residence time of fellow prey and risk factors on the prey average residence time. We assumed that hares and rabbits perceived a high risk with an increase in the fellow prey total residence time. Moreover, we assumed that risk associated with fellow prey total residence time, depended on predator space use and the characteristics of the environment (see Kuijper et al., 2015).

\section{Data analysis}

We investigated prey space use by selecting camera locations that captured the species under investigation on at least one occasion. Furthermore, we selected datasets with camera locations that captured the same species to separate the effects of predators and fellow prey on prey space use (Halliday \& Morris, 2013). This resulted in six non-overlapping datasets to assess prey space use: hare only (21 cameras), rabbit only (36), hare and rabbit (8), hare and fox (18), rabbit and fox 
(55), and hare, rabbit and fox (20). Potential effects of species avoiding camera patches, however closely present to a camera patch, were considered negligible. Additionally, we assumed that the average residence time was not influenced by individuals visiting multiple or recurrently the same camera location. An overview of the characteristics of the response and predictor variables used in the datasets can be found in Appendix 3, table A3.3. Note that the range of risk factors varies over the datasets.

We assessed the average residence time by running linear mixed models (lmer, R Package lme4 version 1.1-12). Each model included the session number as a random factor and was weighted according to the square root of the number of photos of the response species taken at a camera location (Lipták, 1958). We could not use IT criteria (i.e., AICc) to compare models in different datasets, because the selected datasets contained different samples. We thus assessed the relative strength of the parameters using standardized regression coefficients. The hare, rabbit and fox residence times were right skewed and log10 transformed for the analysis. We centred the binary predictor variables (open or half-open vegetation structure, non-edge or edge location) and scaled the continuous predictor variables by dividing their means by two standard deviations (Gelman, 2008). The data points of the standardized predictor variables were within two standard deviations from the mean.

\section{RESULTS}

\section{Effects of predator space use and the environment}

Fox space use (i.e., average residence time) was only correlated with the characteristics of the environment when hares were present. When hares were present, foxes spent more time in edge habitats compared to non-edge habitats $(\mathrm{t}=4.9, \mathrm{n}$ $=36, \mathrm{p}<0.0001)$. In the presence of foxes, hare space use was positively related to the mean shrub height. Hares spent more time in edge habitats compared to non-edge habitats, and hares spent more time in half-open vegetation structures compared to open vegetation structures (table 3.1). In the absence of foxes, rabbits spent more time in half-open vegetation structures compared to open vegetation structures (table 3.2). 


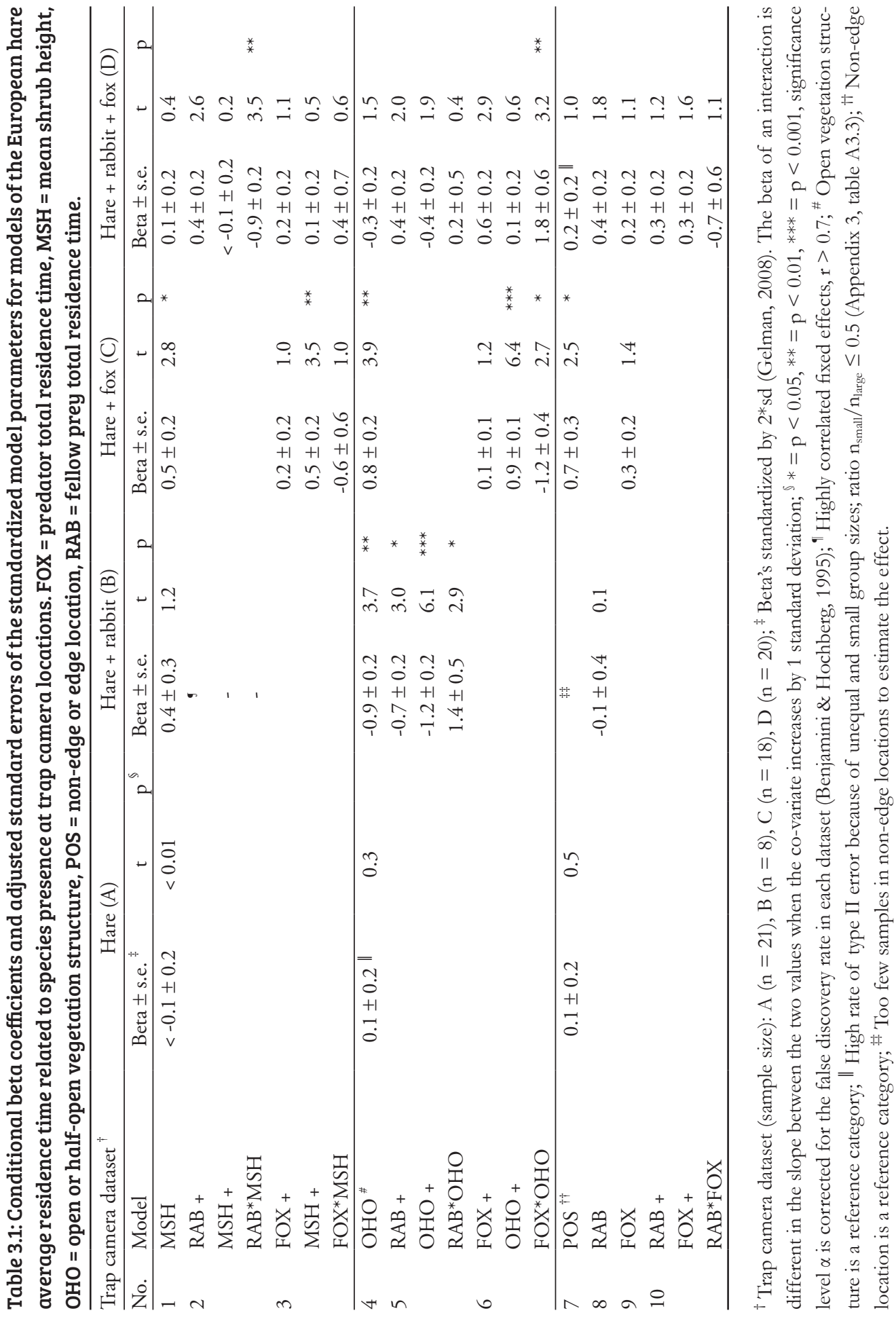



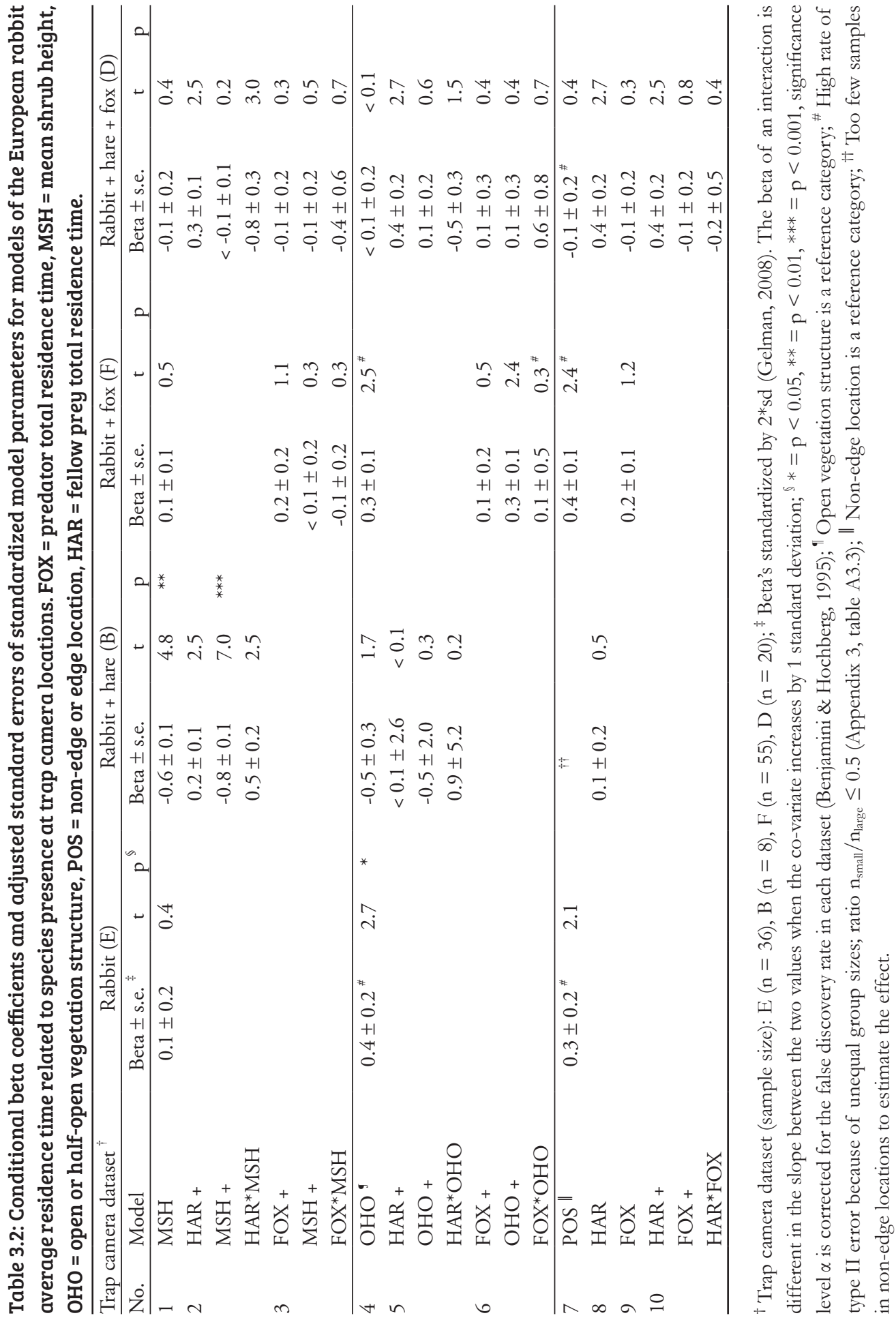
Effects of fellow prey space use

In the presence of only rabbits, hares spent more time in open vegetation structures compared to half-open vegetation structures, whereas this was reversed in the presence of only foxes. In the absence of rabbits, space use by hares was negatively related to space use by foxes in areas with a half-open vegetation structure (figure 3.2), whereas it was unrelated in areas with an open vegetation structure. This interaction reversed in the presence of rabbits (figure 3.3). In the model with rabbit space use as the single predictor variable, space use by hares was uncorrelated to space use by rabbits, with or without a predator present. However, in the presence of foxes, space use by hares was positively related to space use by rabbits in habitats with a low mean shrub height, whereas it was negatively related to space use by hares in habitats with a high mean shrub height (figure 3.4). In the absence of foxes, space use by hares was negatively related to space use by rabbits in open vegetation structures, with no relationship in half-open vegetation structures (figure 3.5).

In the model with hare space use as the single predictor variable, space use by rabbits was uncorrelated to space use by hares, with or without a predator present. Space use by rabbits, however, was negatively related to the mean shrub height, but only when hares were present (table 3.2).

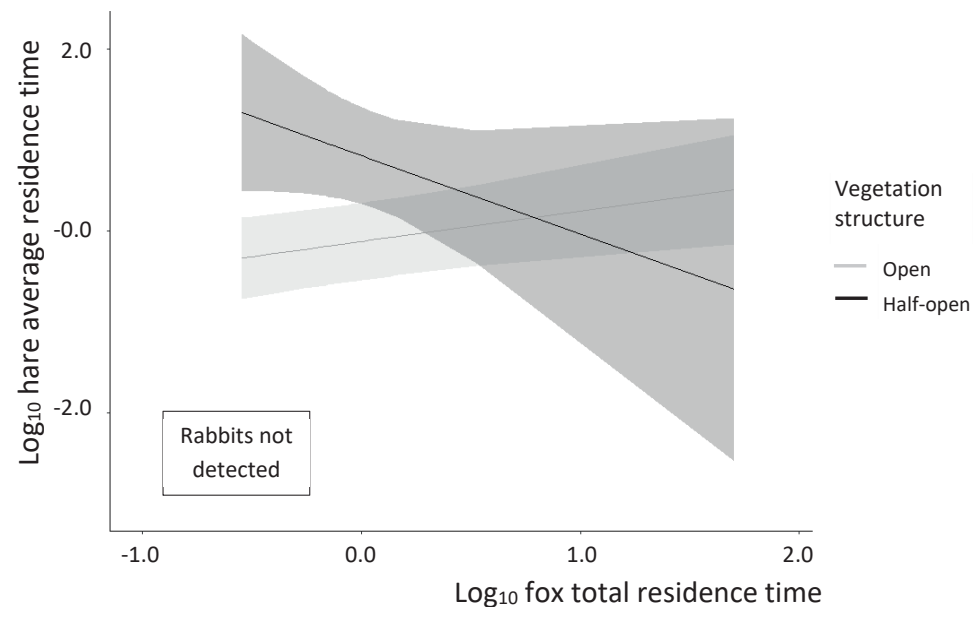

Figure 3.2: Hare average residence time $(\bar{X} \pm 95 \% C I)$ as a determinant of the interaction between the fox total residence time and vegetation structure ( $n=18$, standardized).

Rabbits were not detected by cameras. 


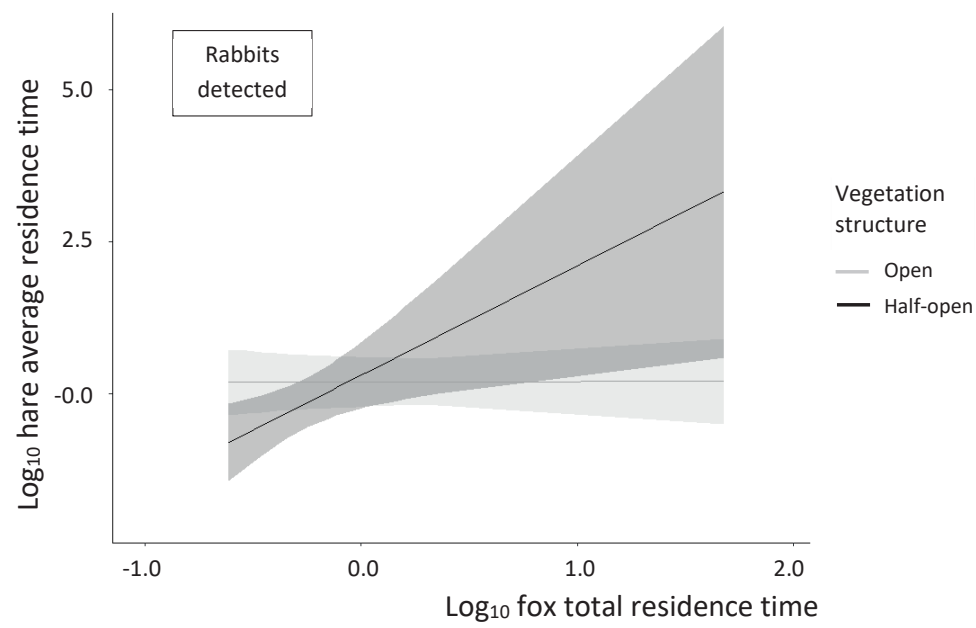

Figure 3.3: Hare average residence time $(\bar{X} \pm 95 \% C I)$ as a determinant of the interaction between the fox total residence time and vegetation structure ( $n=20$, standardized).

Rabbits were detected by cameras.

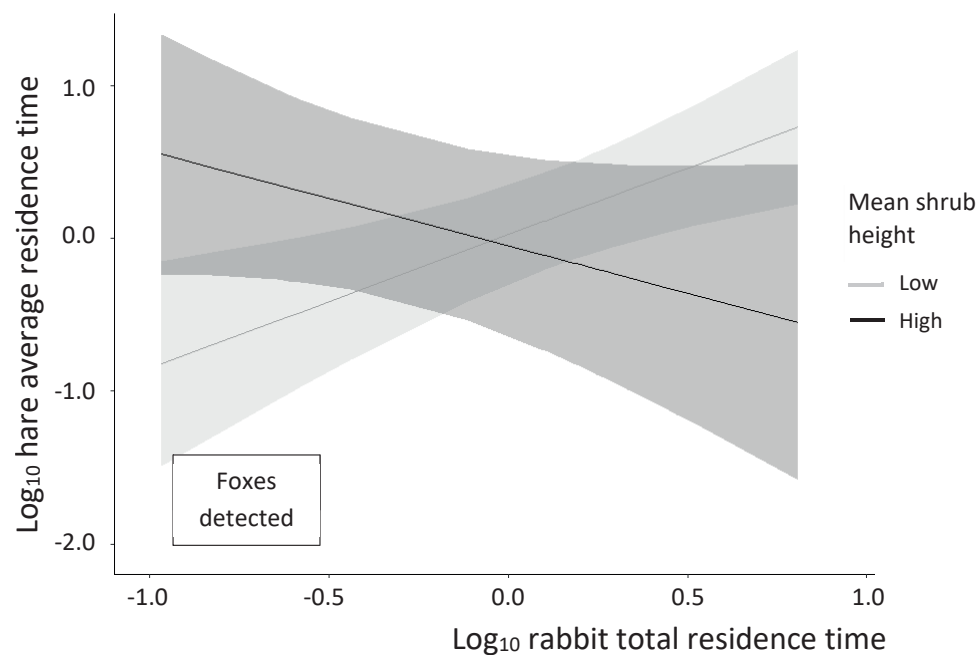

Figure 3.4: Hare average residence time $(\bar{X} \pm 95 \% C I)$ as a determinant of the interaction between the rabbit total residence time and mean shrub height ( $n=20$, standardized).

Foxes were detected by cameras. 


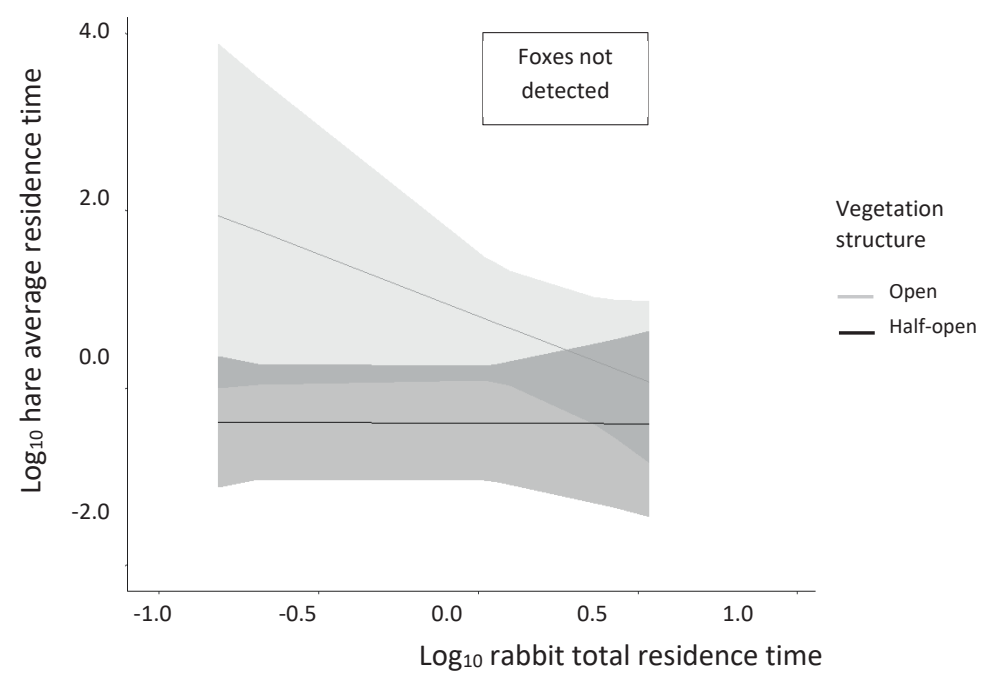

Figure 3.5: Hare average residence time $(\bar{X} \pm 95 \% C I)$ as a determinant of the interaction between the rabbit total residence time and vegetation structure ( $n=8$, standardized).

Foxes were not detected by cameras.

\section{Space use in time}

Spatial or temporal refuges can favour the coexistence of prey species that compete for enemy-free space (Krebs, 2009). Our previous analyses were indifferent to time and investigated space use by predators and prey captured by a set of cameras during a 15-day sampling period. When looking at the same area covered with one camera at a time, however, we found only sixteen incidences where both foxes and rabbits visited the same area in the same hour out of $410(3.9 \%)$ and $2169(0.7 \%)$ detections of fox and rabbit, respectively. We did not record any visits of foxes and hares at the same camera during the same hour, whereas thirteen times both hares (4.7\% out of 277$)$ and rabbits $(0.6 \%)$ visited the same area during the same hour. Although the number of subsequent visits by rabbits and foxes within the same hour was low, the percentage of camera visits (i.e., activity) by rabbits during the 24-hour cycle was significantly correlated to the percentage of camera visits by foxes $(\mathrm{r}=0.76, \mathrm{n}=21, \mathrm{p}<0.001)$ (figure 3.6). Hares responded to a change in species present by shifting the times of their activities. Hares were less active during the period between astronomical dusk and dawn and more active during the rest of the day, when all three species were detected at a camera location (figure 3.7). Moreover, when all three species were detected, hares were active significantly earlier (2.5 hours) during sunrise-sunset compared to camera locations where hares only, hare and rabbit, or hare and fox were present $(\mathrm{t}=3.8, \mathrm{df}=6.7, \mathrm{p}=0.007)$. 


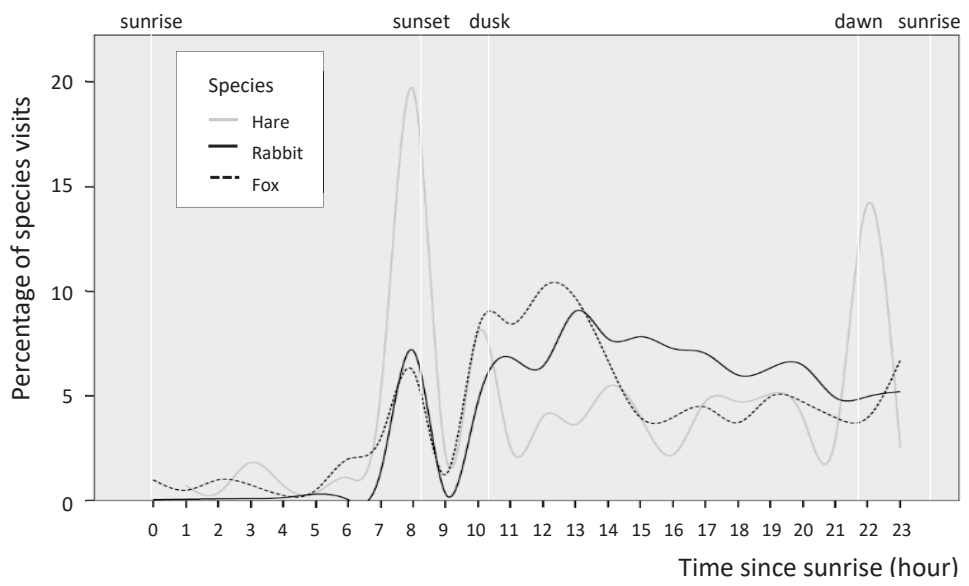

Figure 3.6: Percentage of species visits during a 24-hour cycle beginning at sunrise $\left(\mathrm{n}_{\text {hare }}\right.$ $=275, \mathrm{n}_{\text {rabbit }}=2133, \mathrm{n}_{\text {fox }}=403$ ).

The time since sunrise is corrected for the daily shift in astronomical dusk and dawn at the study site. The periods in the graph are averaged over the study time. Visits were compared by their relative timing within a period.

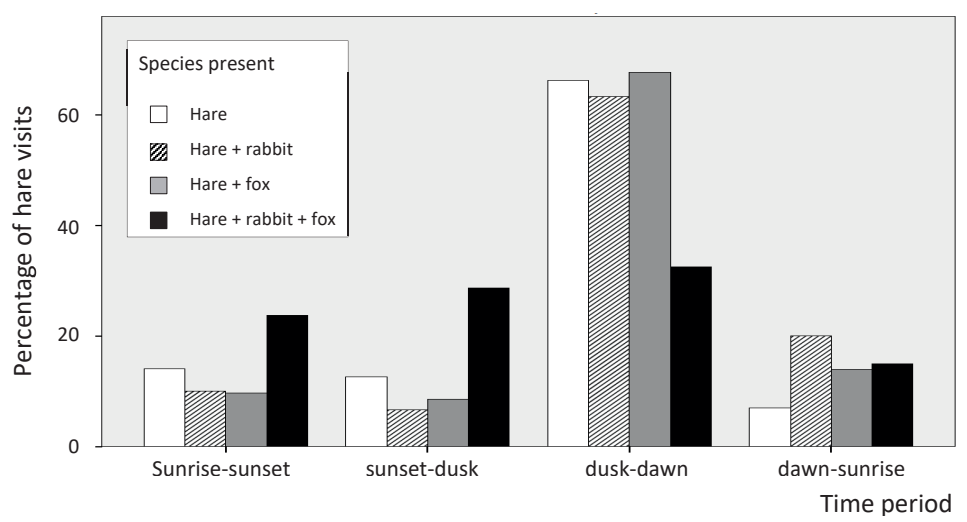

Figure 3.7: Percentage of hare visits per species present during the four time periods $\left(\mathrm{n}_{\text {hare }}=51, \mathrm{n}_{\text {hare }+ \text { rabbit }}=18, \mathrm{n}_{\text {hare }+ \text { fox }}=56, \mathrm{n}_{\text {hare }+ \text { rabbit }+ \text { fox }}=54\right)$.

\section{DISCUSSION}

We explored the effects of predators, fellow prey and risk factors on space use by two sympatric prey species. Few field studies on terrestrial systems have quantitatively investigated the apparent competition between mammal species (Oliver et al., 2009; Johnson et al., 2013). In contrast to former studies, we used direct 
behavioural observations of fine-scaled space use from trap cameras to assess the effects of fellow prey on prey space use.

As expected we observed apparent asymmetric competition. The presence of rabbits reversed the relationship between space use by hares and foxes in half-open vegetation structures. The presence of foxes released the interaction between space use by rabbits and vegetation structures on the space use by hares. Apparent asymmetric competition can result from differences in prey niche overlap and spatial variations in risk factors (DeCesare et al., 2010). Although there is considerable overlap in habitat use between rabbits and hares (Flux, 2008), their habitat-specific escape modes differ markedly. Rabbits use their burrows to escape predation risk (Bakker et al., 2005), whereas hares stand up to predators and can make use of crypsis and flight (Focardi \& Rizzotto, 1999). Niche overlap not only affects apparent competition but also drives competition. Hares and rabbits seem to avoid each other in open vegetation structure and tall shrubs. This behaviour can be interpreted as apparent competition, but could likewise be the result of competition for space or food (Halliday \& Morris, 2013). However, populations of small- and medium-sized prey species, as our hares and rabbits, are suggested to be strongly determined by predation instead of food limitation (Sinclair et al., 2003). Moreover, competition for food is predicted to dominate in landscapes with low resource availability (Chesson \& Kuang, 2008), whereas predator avoidance is predicted to dominate in landscapes with high resource availability (Hopcraft et al., 2010), which could be the case in our study site.

Hares and rabbits have a considerable dietary overlap (Kuijper et al., 2004), and Homolka (1987) classified them as trophic competitors when sympatric (but see Katona et al., 2004). Although we found no indication of interference competition, avoidance between hares and rabbits in open vegetation structures, as in our data, could be the result of exploitative competition. Rabbits maintain highquality patches with low vegetation heights (Bakker et al., 2005). Intense grazing by rabbits can change the plant species composition, vegetation height and perceived predation risk, thereby affecting the interaction between the two species and resulting in competition or facilitation (Shipley, 2007; Bakker et al., 2009). For example, a change in the plant species composition (Whinam et al., 2014) could lead to an increase or decrease in preferred food plants available for hares (Kuijper \& Bakker, 2008). Moreover, the interspecific interaction between hares and rabbits may also be affected by differences in body size (Prins \& Olff, 1998), feeding style, digestive system and morphology (Bell, 1971). Hares are twice as large as rabbits and have a relatively larger bite size. In comparison to rabbits, hares forage less efficiently on short vegetation and require taller vegetation to obtain 
their absolute daily energy requirements (Shipley, 2007). Habitat modification by rabbits could reduce hare foraging efficiency, leading to exploitative competition. Hence, this can explain our observation that hares and rabbits seem to avoid each other in open vegetation structures.

When predators are present and evasion of risky locations will cost more time, larger animals cannot afford to select higher quality forage (Shipley, 2007). The presence of foxes possibly released the avoidance between hares and rabbits and promoted their coexistence (DeCesare et al., 2010) in open vegetation structures with high risk. Nevertheless, it seemed that the avoidance in tall shrubs with low risk was not released, possibly because of the thermoregulatory benefits for the hare (Tapper \& Barnes, 1986) and presence of rabbit burrows (Bakker et al., 2005). At our study sites, hares and rabbits seem to coexist, probably because of a shared generalist predator (Bonsall \& Hassell, 2000), apparent competition, spatial variation in risk factors (i.e., refuges, Bonsall et al., 2005) and segregation in time.

Relative to hares, space use by rabbits was not correlated to space use by foxes, space use by fellow prey, or risk factors. As a central place forager, rabbits are less capable of shifting their use of space as a result of predation risk. Non-centralplace foragers like hares, however, are more capable of shifting their use of space, as they have larger home ranges, have access to a wider range of food resources (Stott, 2007), and possess multiple escape modes (Wirsing et al., 2010). Moreover, hares have a relatively small digestive system, which acts as a weight-minimizing adaptation to enhance flight (Stott, 2007) and maximizes the passage rate to cope with low-quality forage (Kuijper et al., 2004). Therefore, hares can compensate for a poorer diet that comes at the cost of a shift in space (Laundré et al., 2010).

\section{Conclusions}

Hares and rabbits responded differently to space use by foxes, space use by fellow prey, risk factors and the presence of other species. Space use by hares was correlated to space use by foxes and to space use by fellow prey, both of which interacted with the risk factors. Hare activity, however, was uncorrelated to fox activity in time. Rabbit activity in time was strongly correlated to fox activity, whereas space use by rabbits was uncorrelated to space use by foxes.

In the presence of predators, hares that were affected by fellow prey, shifted habitat and sought low-risk locations. Predator presence possibly released avoidance and promoted coexistence between our two sympatric prey species in the high-risk habitat, but had no effect in the low-risk habitat. Apparent competition 
may have reversed the relationship between the space use by predators and risk factors on the space use by prey. Our study demonstrates that space use of two sympatric prey species is complex. The properties of prey species that affect the relations between the space use by predators, space use by fellow prey and risk factors should be taken into account. These prey species' properties include the escape mode, body size with related dietary requirements, and dependence or independence of prey to a central place. The presence of fellow prey, predators or both can determine the behaviour of the prey species. Additionally, risk factors interact with the space use by predators or the space use by fellow prey on the space use by prey.

\section{APPENDIX 3}

Table A3.1: An overview of the vegetation types of camera locations in the study area.

\begin{tabular}{cll}
\hline No. & Vegetation types $^{1}$ & \# cameras \\
\hline 1 & Calcareous dune grassland & 66 \\
2 & Dune grasslands & 44 \\
3 & Burnet rose, creeping willow-, blackberry thicket & 24 \\
4 & Flower rich grasslands & 21 \\
5 & Thickets & 6 \\
6 & Deciduous forest & 4 \\
7 & Near-shore communities & 4 \\
8 & Calcareous dune valley & 2 \\
9 & Nutrient rich grasslands & 2 \\
10 & Non-calcareous dune grassland & 1 \\
11 & Remaining forest & 1 \\
12 & Reed swamp & 1 \\
13 & Reed swamp communities & 1 \\
\hline & & total \\
\hline
\end{tabular}

${ }^{1}$ Based on an overlay between the camera locations and a high resolution (1:5000) GIS vegetation map by Everts et al. (2008; 2009). 


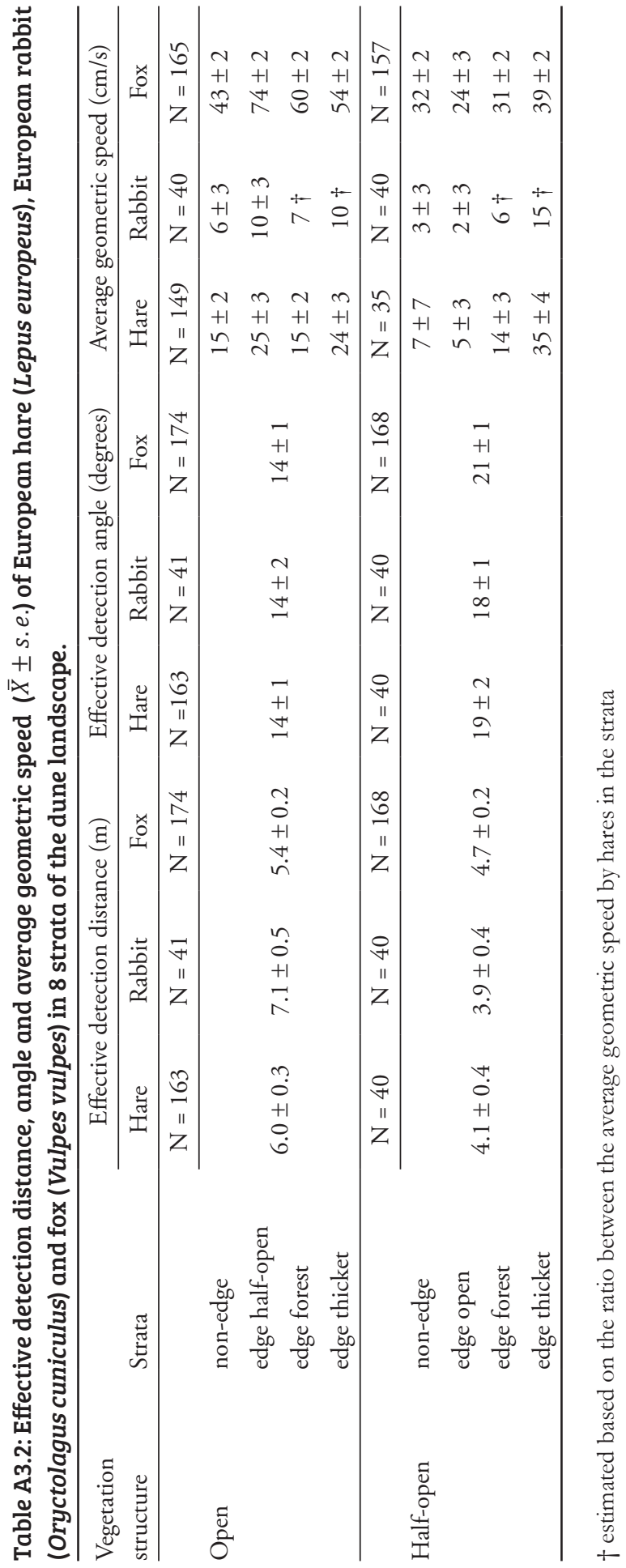




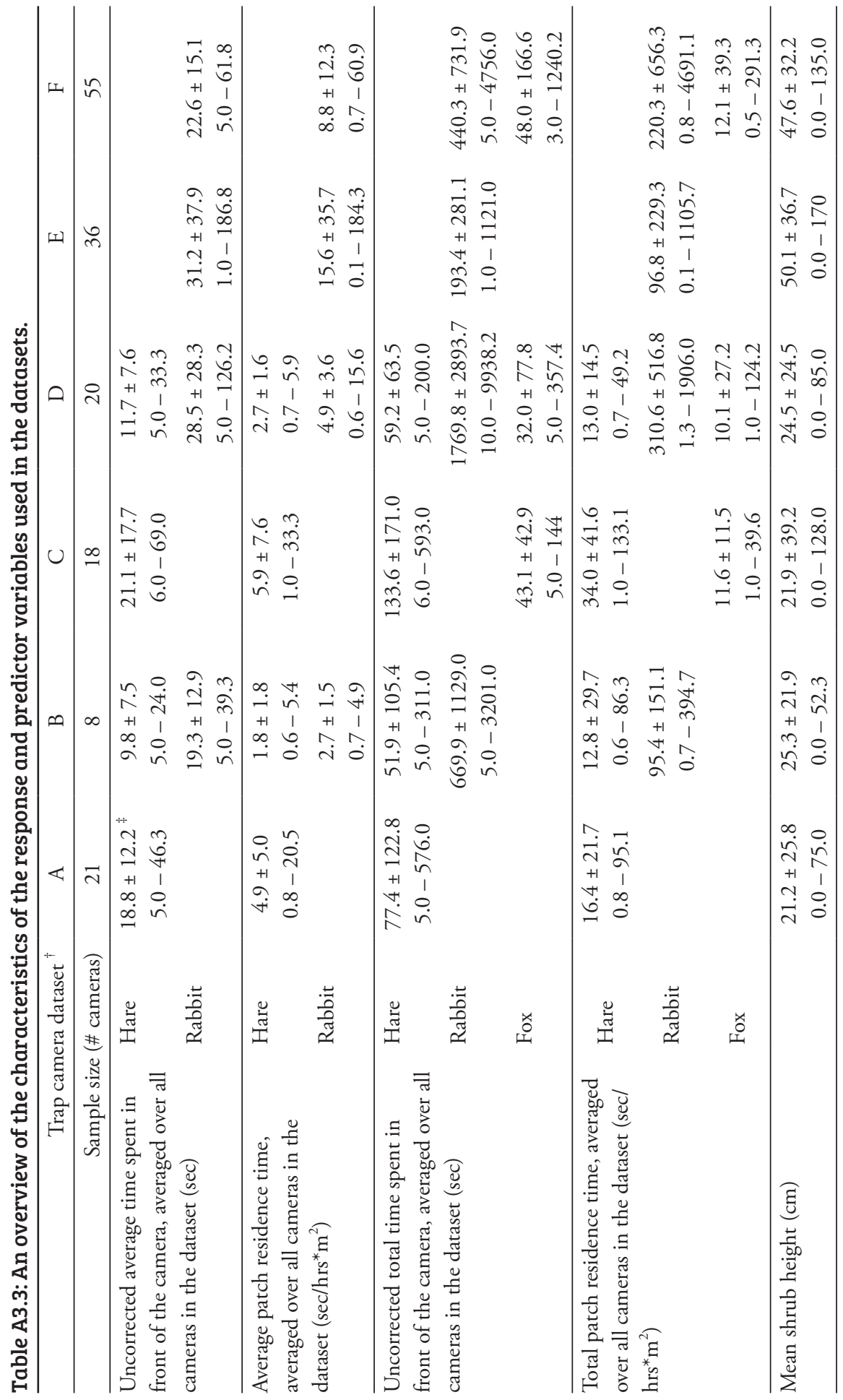




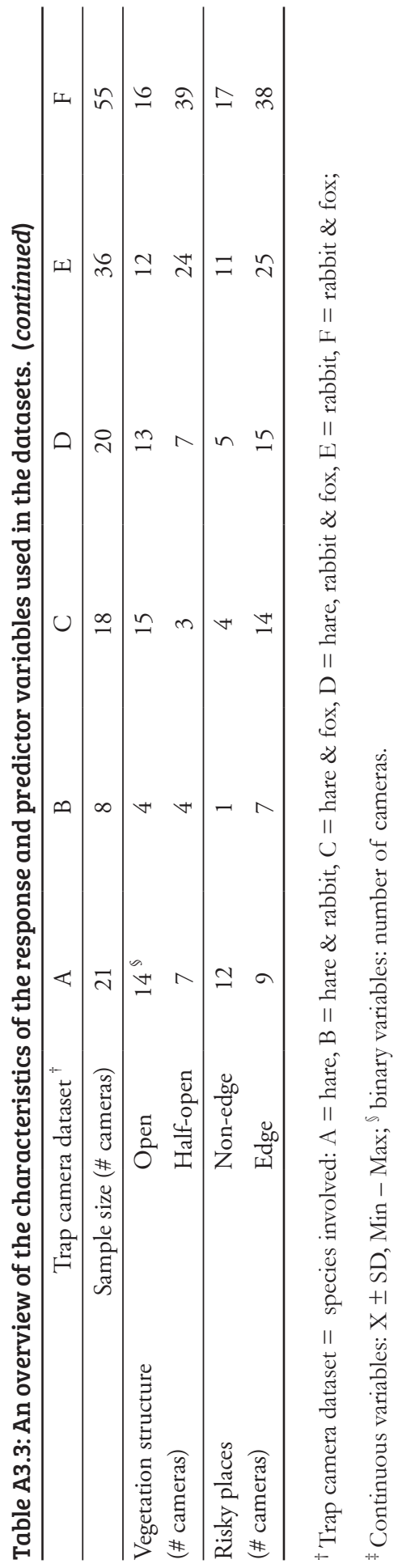





\section{CHAPTER 4}

Food quality and quantity is more important explaining foraging of an intermediate-sized mammalian herbivore than predation or competition

Weterings, M.J.A., Moonen, S., Prins, H.H.T.,

Van Wieren, S.E., Van Langevelde, F. 


\section{ABSTRACT}

During times of high activity by predators and competitors, herbivores may be forced to forage in patches of low-quality food. However, foraging in patches of low-quality food will come at a cost of increased search and handling times. Nevertheless, the relative importance in determining where and what herbivores forage still remains unclear, especially for small and intermediate-sized herbivores.

Our objective was to test the relative importance of predator and competitor activity, and forage quality and quantity for the proportion of time spent in a vegetation type and the proportion of time spent foraging by the intermediatesized herbivore European hare (Lepus europaeus).

We investigated the time spent at a location and foraging time of hare using GPS with accelerometers. Forage quality and quantity was analysed based on hand-plucked samples of a selection of the locally most important plant species in the diet of hare. Predator and competitor activity was investigated using a network of camera traps.

Hares spent a higher proportion of time in vegetation types that contained a higher percentage of fibres (i.e., NDF). Besides, hares spent a higher proportion of time in vegetation types that contained relatively low quantity and quality of forage (i.e., high percentage of fibres) during days that foxes (Vulpes vulpes) were more active. Also during days that rabbits (Oryctolagus cuniculus) were more active, hares spent a higher proportion of time foraging in vegetation types that contained a relatively low quality of forage.

Overall, food quality and quantity more strongly affected hare foraging time than the activity of predators, and the activity of smaller competitors was least important. Although small and intermediate-sized herbivore behaviour or habitat use is predicted to be controlled by predation risk (i.e., landscape of fear), our study shows that food quality and quantity is more important. It seems that we need to reappraise the importance of the landscape of food in a world of fear. 


\section{INTRODUCTION}

Decisions of animals about where and what to eat depend on the outcome of the costs and benefits of foraging (Robbins, 1993). Costs include searching and handling time of the food, the risk of predation (i.e., landscape of fear sensu Laundré et al., 2001) and the effects of competitors (Pays et al., 2012), whereas benefits are related to survival or reproduction (Barboza et al., 2009). Mammalian herbivores are predicted to select food patches that optimize intake rate (i.e., forage quantity) or digestible intake (i.e., forage quality) given these costs (Shipley, 2007). Especially for small and intermediate-sized mammalian herbivores, the trade-off between predation and food intake is important as on the one hand these herbivores have low absolute nutritional requirements but need highly digestible food compared to large herbivores. As high-quality food is often scarce, these herbivores have to spend a lot of time searching for patches with high-quality food. On the other hand, small and intermediate-sized herbivores are more vulnerable for predation than larger ones (Sinclair et al., 2003; Thaker et al., 2011). Although mass-specific nutritional requirements increase with declining body mass (Demment \& Van Soest, 1985), populations of small and intermediate-sized herbivore species are suggested to be more strongly determined by predation than by food limitation (Sinclair et al., 2003; Brown \& Kotler, 2004; Hopcraft et al., 2010). For this group of species the landscape of fear dominates the landscape of food. In times of high risk, small and intermediate-sized herbivores may therefore be forced to forage in patches of low-quality food (Hernández \& Laundré, 2005) instead of foraging in patches of high-quality food, because they have less time to search for the scarce and small patches of high-quality food (Shipley, 2007), or because travelling between small patches of high-quality food increases the probability of detection by predators (Broom \& Ruxton, 2005; Eccard \& Liesenjohann, 2014). Moreover, patches that contain low-quality food (often tall vegetation) offer more cover for prey at risk (Riginos \& Grace, 2008). If predators force small and intermediate-sized herbivores to seek cover in patches of low-quality food, then these herbivores must spent more time foraging, because of the increased search and handling times, than in patches of high-quality food (Heuermann et al., 2011).

Foraging of herbivores can also be negatively affected by the presence of competitors (Focardi et al., 2006; Ferretti et al., 2015). Interactions between herbivores and their competitors can be affected by differences in body mass, feeding style, digestive system and morphology (Bell, 1971). Similarity in body mass and morphology is expected to increase competition, whereas differences in 
body mass and morphology allow habitat segregation between herbivores (Prins \& Olff, 1998). For example, the bite size of smaller-sized herbivores allows higher intakes of high-quality food on grasslands that contained a lower quantity of food relative to larger-sized herbivores (Wilmshurst et al., 2000). Consequently, intermediate-sized herbivores can be excluded by smaller herbivores if densities of smaller competitors are high, plant biomass is low, and food becomes depleted and unavailable (Shipley, 2007). For intermediate-sized herbivores, locations with high-quality food are then hypothesized to be traded for locations with low-quality food during times of high competitor activity by smaller herbivores.

While the importance of predation risk (Lima \& Dill, 1990), competition (Prins \& Olff, 1998; Arsenault \& Owen-Smith, 2002), and forage quality and quantity (Barboza et al., 2009) for foraging time has been recognised widely, their relative importance in determining where and what intermediate-sized herbivores forage remains unclear (Arsenault \& Owen-Smith, 2002; Brown \& Kotler, 2004; Morris, 2009). Whereas many studies focus on the trade-off between resource acquisition and predation (Sih, 2005; Laundré, 2010; Thaker et al., 2011), few studies simultaneously consider the trade-off with competition (Lima, 1998; Morris, 2002; 2009). It has been hypothesized that the effect of intra- and interspecific competition on foraging behaviour is more important than the effect of predation risk (Grand \& Dill, 1999a; Halliday \& Morris, 2013), especially if resource availability is low (Chesson \& Kuang, 2008), and herbivores are similar-sized (Sinclair, 1985). Our objective was therefore to test the relative importance of predator and competitor activity, and forage quality and quantity for the proportion of time spent in a vegetation type and the proportion of time spent foraging by the intermediatesized herbivore European hare (Lepus europaeus).

We expected that during times that predators and smaller competitors are more active, intermediate-sized herbivores spent more time in vegetation types that contain lower food quality (Prins \& Ollf, 1998; Wilmshurst et al., 2000; Shipley, 2007), and therefore they must spend more time on foraging. Whereas during times that predators or smaller competitors are less active, intermediate-sized herbivores spend more time in vegetation types that contain higher food quality and therefore they could spend less time on foraging. We hypothesized that time spent foraging by intermediate-sized herbivores is more strongly affected by competitor activity than by predator activity (Halliday \& Morris, 2013), forage quality or forage quantity (Sinclair et al., 2003; Hopcraft et al., 2010). 


\section{METHODS}

We conducted the study in the coastal-dune landscape Noordhollands Duinreservaat' near Castricum $\left(52^{\circ} 33^{\prime} \mathrm{N}, 4^{\circ} 38^{\prime} \mathrm{E}\right)$ in the Netherlands. Three areas, Castricum (ICAS) (325 ha), Vennewater (VW) (275 ha) and Koningsbos (KB) (50 ha), were selected based on previous sightings of hare. The coastal-dune landscape on nutrient-poor sandy soils contained a mosaic of 20 dune vegetation types relevant for hares (Appendix 4, table A4.1). Red fox (Vulpes vulpes), as predator of hare, was present at a high density of 5 ind. $\mathrm{km}^{-2}$, whereas the European rabbit (Oryctolagus cuniculus), as smaller competitor, was present at a low density of 2 ind. $\mathrm{km}^{-2}$ (Mulder, 2005).

\section{Hare foraging behaviour and location}

To measure the time spent foraging, we tracked 12 hares in the study area between 15 October 2014 and the first of January 2015. During this period female hares store energy, because they are capital breeders, especially when having their first litter (Valencak et al., 2009). We therefore expected female hares to be more selective in their foraging behaviour, even more because the nutrient quality of the vegetation during the study period is relatively low (Smith et al., 2005).

Hares were flushed by a line of beaters and caught using Speedset static hare nets (height $45 \mathrm{~cm}$, with $13 \mathrm{~cm}$ full mesh; JB's Nets, Alexandria, U.K.). Caught hares were quickly removed from the nets, blindfolded (Paci et al., 2012) and temporarily kept in darkened wooden boxes to reduce stress. Healthy hares were tagged without sedation (Gerritsmann, et al., 2012) immediately after all hares in an area were flushed. Hares were equipped with a neck belt that contained a GPS and an accelerometer ( $69 \mathrm{~g}, 1.8 \pm 0.2 \%$ of body weight) with wireless communication (Type A, E-obs GmBH, Gruenwald, Germany). After tagging, we measured body weight ( $\overline{\mathrm{X}} \pm \mathrm{SD}, 3719 \pm 281 \mathrm{~g})$, and determined sex (7 females, 4 males, 1 unknown) and age ( 6 individuals $<1$ year old, $5>1$ year, 1 unknown) of the hares. The capturing of hares was executed under the approval of the Wageningen University Animal Experiment Committee (no. 2014034.b) and followed the EU Directive $2010 / 63$ on the protection of animals used for scientific purposes.

Hares were allowed to settle down for a period of five days after capturing before the GPS and accelerometer started recording data (Petrovan et al., 2013). The GPS position of individual hares was recorded every 12 minutes, 24 hours a day. Acceleration in three axes was recorded every minute for eight seconds, 24 hours a day, with a frequency of $31.62 \mathrm{~Hz}$, allowing detailed determination of 
behaviour. The raw data of accelerometer recordings was transformed into physical units $\left(\mathrm{m} . \mathrm{s}^{-2}\right)$ by:

Eq.1:

$$
a_{i}=\left(n_{i}-n_{i, \text { zerog }}\right) \cdot c_{i} \cdot g
$$

where $a_{i}\left(m \cdot s^{-2}\right)$ is the acceleration of axis $i, n_{i}$ is the raw data (unit-less values) of one axis, $\mathrm{n}_{\mathrm{i} \text {, zerog }}$ is the raw data without gravitational force and no dynamic acceleration (unit-less value), $c_{i}$ is a constant (unit-less value), and $g$ is the acceleration caused by earth gravitation $\left(9.81 \mathrm{~m} . \mathrm{s}^{-2}\right)$. The constants $\mathrm{c}_{\mathrm{i}}$ and $\mathrm{n}_{\mathrm{i}, \mathrm{zerog}}$ of each accelerometer were calibrated and measured before the start of the study.

For each one-second segment of acceleration, we calculated the following parameters for each hare (Nathan et al., 2012; Bom et al., 2014):

A) For each axis separately: 1) standard deviation of the static acceleration, 2) maximum dynamic acceleration component, 3) arithmetic mean of the smoothed time series (moving median with window size $\mathrm{k}=5$ ), 4) skewness, and 5) kurtosis.

B) For all three axes combined: 1) the resultant of the $x, y$ and $z$ axis of the parameters described at $A$ ), as the square-root of the sum-of-squares of the three axes, 2) dynamic body acceleration, and 3) overall dynamic body acceleration (ODBA).

To label the accelerometer data with behaviours, we recorded 8771 seconds of behaviour (range: 3-4122 s, $\mathrm{n}=8$ ) using a handheld video of tagged hares in coastal-dune landscapes. Video fragments were labelled with one of 8 types of behaviour (laying, sitting, sitting alert, grooming, scratching, chewing, foraging and movement) using the software Avidemux (2.6.6). Only one-second segments that contained $100 \%$ of the same behaviour were used in the subsequent analysis. Decision tree software (AcceleRater, Resheff et al., 2014) together with the labelled accelerometer segments were used to classify the unlabelled accelerometer data into foraging (precision: $83 \%$, accuracy: 92\%, recall: 93\%).

\section{Forage quality and quantity}

We used a high resolution GIS map (1:5.000) of vegetation types in the study area (Everts et al., 2008; 2009) to extract the vegetation types for the corresponding GPS locations of hares. Forage quality and quantity were estimated in the vegetation types that were used by the tracked hares. We measured quantity (edible biomass) and quality (concentration of nutrients) of the vegetation as forage for the hares in the vegetation types based on a selection of the locally most impor- 
tant plant species in the diet of hares, namely: Festuca rubra, Agrostis capillaris, Poa pratensis, Holcus lanatus, Poa trivialis, Taraxacum officinale, Rubus caesisus (Kuijper et al., 2008; professional judgement S.E. van Wieren) and a commercial flower bulb species.

For each plant species, we hand-plucked mixed samples of edible biomass, i.e., green plant parts that have a high nutritional value and are selected by hares (Homolka, 1987), in 6 randomly placed circular plots (10 m radius) in each vegetation type. In each vegetation type, we visually estimated the percentage cover of each plant species in six 2x2 m quadrants (using 40x40 cm sub quadrants of the $2 \times 2 \mathrm{~m}$ quadrants) and measured their average height at 5 orthogonal locations. We assumed plant parts at more than $50 \mathrm{~cm}$ from ground level were unavailable as forage for the hares. For each plant species, we estimated the conversion factor between the total biomass, edible biomass and the volume of the plant species by removing all vegetation in two $50 \times 50 \mathrm{~cm}$ quadrants, i.e., one with the highest and one with the lowest average height of the plant species.

To assess changes in forage quality and quantity over the research period, mixed samples of edible biomass of each plant species were collected in two sample sessions (Oct \& Jan). Plant parts were air-dried, stored and chemically analysed for the percentage of N, P, Ca, and NDF (Neutral Detergent Fibre) in the biomass. Because the amount of fibre in the vegetation can reduce food intake and affect foraging behaviour, especially for small herbivores that generally avoid vegetation types with high fibre content, we measured NDF as an index of plant fibre content (i.e., total cell walls) (Barboza et al., 2009). We did not find any changes in the forage quality and quantity of the vegetation types between the two sample sessions. For each nutrient, average concentration of each vegetation type was calculated by averaging the percentage of nutrients for each plant species present in the vegetation type, weighted by their volume per square meter up to $50 \mathrm{~cm}$ in height. We calculated the average forage quantity $\left(\mathrm{g} \cdot \mathrm{m}^{-2}\right)$ for each vegetation type by summing the amount of edible biomass (g) of all plant species in one square meter of the vegetation type up to $50 \mathrm{~cm}$ in height.

The average nutrient and plant fibre concentrations of the vegetation types were highly correlated (Appendix 4, table A4.1). We therefore extracted two PCA axes of the nutrients ( $\%$ of $\mathrm{N}, \mathrm{P}, \mathrm{Ca})$ and the fibre content ( $\%$ of $\mathrm{NDF}$ ) by a principal component analysis (SPSS version 23.0). Axes were rotated by a Varimax with Kaiser Normalization. Factor scores above 1 (Kaiser, 1960) were calculated and standardized by the Anderson-Rubin method (DiStefano et al., 2009), which 
ensures orthogonality of the estimated factors. The first PCA axis was strongly positively correlated with the percentage of $\mathrm{N}$ and $\mathrm{P}$ in the edible biomass of the vegetation (table 4.1). The second PCA axis was strongly positively correlated with the percentage of NDF and strongly negatively correlated with the percentage $\mathrm{Ca}$ in the edible biomass of the vegetation. We multiplied the $2^{\text {nd }}$ PCA axis by -1 to get a consistent interpretation of forage quality, because we associated poor forage quality with a higher percentage of NDF.

Table 4.1: Rotated PCA component coefficient values of forage quality of the vegetation types in the coastal-dune landscape $(n=20)$. Note the multiplication of PCA axis 2 with -1 to get a consistent interpretation of forage quality.

\begin{tabular}{|c|c|c|}
\hline \multirow[b]{2}{*}{$\begin{array}{l}\text { Nutrients } \\
\text { and NDF }\end{array}$} & \multicolumn{2}{|c|}{$\begin{array}{c}\text { Forage quality }{ }^{1} \\
\text { (\% nutrients in edible biomass) }\end{array}$} \\
\hline & $\begin{array}{c}\text { QL1 = PCA axis } 1 \\
69.7 \%(2.8)^{3}\end{array}$ & $\begin{array}{c}\text { QL2 }=-1 * \text { PCA axis } 2 \\
27.7 \%(1.1)\end{array}$ \\
\hline $\mathrm{N}$ & 0.96 & -0.13 \\
\hline $\mathrm{P}$ & 0.96 & -0.21 \\
\hline $\mathrm{Ca}$ & -0.55 & 0.83 \\
\hline NDF & 0.04 & -1.00 \\
\hline
\end{tabular}

${ }^{1}$ Varimax with Kaiser Normalization; list-wise deletion, PCA components $>0.6$ are bold; ${ }^{2}$ NDF $=$ neutral detergent fibre on ash-in-basis; ${ }^{3}$ Percentage of variance explained by component (eigenvalue of component).

\section{Predator \& competitor activity}

We investigated predator and competitor activity using a network of camera traps that covered 13 vegetation types. Forty-two camera traps (Reconyx Hyperfire: HC500 and HC600, infrared trigger) were randomly placed in open and half-open vegetation (208 camera locations) in the study area for about 15 days between October 16, 2014, and January 8, 2015. Camera traps were interspaced $>50 \mathrm{~m}$ (> $92 \%),>25 \mathrm{~m}$ from waterbodies and $>16 \mathrm{~m}$ from recreational paths, and set up according to the protocol of Jansen et al. (2014). Open vegetation structure has often a high forage quality for hares (Kuijper et al., 2008) where they can easily spot predators, whereas half-open vegetation structures provide lower forage quality, but visual cover (Neumann et al., 2011).

Camera traps were configured to record a burst of ten photos when triggered, without any time lapse between bursts. Visits were visually assessed from sequences of photos, and were assumed to be independent if the quiet period in the beginning was longer than 120 seconds. Overall predator and competitor activity 
was quantified as the total number of camera visits by predators or competitors in the study area during a day.

\section{Data analysis}

We investigated the effects of predator and competitor activity and forage quality and quantity with their interactions on 1) the proportion of GPS fixes in a vegetation type and on 2 ) the proportion of time spent foraging in a vegetation type. We ran multiple generalized linear mixed models in $\mathrm{R}$ (glmer, package lme4 version 1.1-13) for both analyses, with a binomial error structure and logit link. The total number of GPS fixes on a day, and the total number of seconds of measured hare foraging time spent recorded on a day was set as the upper limit of the binomial structure. Predictor variables were grouped into five categories, namely: predator activity, competitor activity, forage quality, forage quantity and vegetation height. We included the average vegetation height as an indicator for prey cover (Verdolin, 2006). Forage quality and vegetation height are often interpreted to be inversely related to each other (see e.g., Bell, 1971). In our study, however, forage quality was measured in the edible biomass only, up to $50 \mathrm{~cm}$ of height. Nevertheless, plant fibre concentration ( $2^{\text {nd }}$ PCA component $)$ and vegetation height were moderately correlated $(\mathrm{r}=-0.58, \mathrm{p}<0.01, \mathrm{n}=20)$, whereas plant nutrient concentration ( $1{ }^{\text {st }}$ PCA component) and vegetation height were not correlated $(\mathrm{r}=-0.12, \mathrm{p}=0.62, \mathrm{n}=20)$. Predator and competitor activity, edible biomass and vegetation height were standardized and scaled by dividing their mean by two standard deviations (Gelman, 2008). Multicollinearity of continuous predictor variables was assessed (Zuur et al., 2010). The Variance Inflation Factor (VIF) of all continuous predictor variables remained below 2.1 in both analyses.

Candidate models were used to assess the relative strength of our hypotheses following Grueber et al. (2011). We generated 24 candidate models from the combinations of the five categories of predictor variables, including an intercept-only model.

Candidate models to explain the proportion of GPS fixes in a vegetation type (Appendix 4, table A4.2) included date as random factor. Date was also used as the repeated measurement variable for each vegetation type. There was no autocorrelation between dates (first five days: $\bar{X} \pm S D, r=0.15 \pm 0.13$ ). All candidate models included area size of the vegetation type as control variable. We excluded vegetation types for which we had no data on forage quality and quantity, and we excluded records when there was no activity of predators or competitors to create a dataset without missing values, for which candidate models could be compared by the small sample Akaike information criterion (AICc). 
Candidate models to explain the proportion of time spent foraging in a vegetation type (Appendix 4, table A4.3) included area, date, and hare-ID in a specific vegetation type as random factors. Hare-ID in a specific vegetation type was nested within date that was nested within area. Date was used as the repeated measurement variable. There was no autocorrelation between dates (first five days: $\overline{\mathrm{X}} \pm \mathrm{SD}, \mathrm{r}=-0.04 \pm 0.04$ ). All candidate models included area type and the sex of the animals as control variables. Body weight did not improve the fit of the models and was left out as a control variable. We excluded the hare of unknown sex to create a dataset without missing values, for which candidate models could be compared by AICc.

We assessed the relative weights of the parsimonious models only, and removed complex models with higher values of AICc that had more predictor variables than the parsimonious ones. Over dispersion of models was assessed by the Pearson Chi-square over the residual degrees of freedom of the model (Crawley, 2007). Assumptions were verified by visual inspection of residuals plotted against the predicted (full model) and identifying outliers with Cook's Distance. Finally, we performed full model averaging of all the parsimonious models to estimate the beta's $(\beta)$ and the (conditional) average standard errors $\left(\widehat{\left.\mathrm{SE}_{\beta}\right)}\right.$ of model parameters.

\section{RESULTS}

We found that the proportion of time that hares spent in a vegetation type was best explained by the model that included the interaction between fox activity and forage quality ( $2^{\text {nd }}$ PCA component), the interaction between fox activity and forage quantity (the amount of edible biomass), and the interaction between fox activity and vegetation height (Appendix 4, table A4.2). The top model had a total relative weight of $87 \%$, and thus had the best fit to our data. Models that included rabbit activity, or the first PCA component of forage quality ( $\mathrm{N}$ and $\mathrm{P}$ ) received very low relative model weights.

Forage quality ( $2^{\text {nd }}$ PCA component) was negatively correlated with the proportion of time spent in a vegetation type (table 4.2). Hares spent a less time in vegetation types that contained a higher percentage of $\mathrm{Ca}$, whereas hares spent more time in vegetation types that contained a higher percentage of NDF. This effect became stronger with increasing vegetation heights (figure 4.1a). The coefficient of fox activity on the proportion of time spent in a vegetation type was positively related to vegetation height (figure $4.1 \mathrm{~b}$ ), but negatively related to forage quantity 
(figure 4.1c) and forage quality ( $2^{\text {nd }}$ PCA component) (figure 4.1d). During days that foxes were more active, hares thus spent a higher proportion of time in tall vegetation types, and vegetation types that contained a relatively low quantity and quality of forage. We found no interaction between rabbit activity and forage quality, or between rabbit activity and forage quantity on the proportion of time spent in a vegetation type. The standardised coefficients show that forage quality or quantity more strongly affected the proportion of time hares spent in a vegetation type than the activity of foxes.

Table 4.2: Results of full-model conditional averaging of all parsimonious generalized linear mixed models on the effect of predator activity and its interaction with forage quality, quantity and vegetation height on the proportion of GPS fixes of European hares in a vegetation type.

\begin{tabular}{lcccccc}
\hline Variables $^{\mathrm{A}}$ & $\begin{array}{c}\text { Estimate } \\
(\beta)^{\mathrm{B}}\end{array}$ & $\begin{array}{c}\text { (conditional) } \\
\overline{\left(\mathrm{SE}_{\beta}\right)}\end{array}$ & $\mathrm{Z}$ value & $2.5 \%-97.5 \%$ C.I. & Effect & $\mathrm{W}_{\mathrm{p}}{ }^{\mathrm{D}}$ \\
\hline Intercept & -3.77 & 0.24 & 15.8 & $-4.24--3.30$ & $\bullet \bullet \bullet$ & 1.00 \\
EB & 0.58 & 0.43 & 1.3 & $-0.27-1.43$ & & 0.88 \\
$\mathrm{VH}$ & 0.88 & 0.63 & 1.4 & $-0.36-2.12$ & & 0.96 \\
QL2 & -0.72 & 0.28 & 2.5 & $-1.27--0.16$ & $\bullet$ & 0.90 \\
QL2*VH & -1.24 & 0.58 & 2.1 & $-2.37--0.10$ & $\bullet$ & 0.08 \\
Fox & -0.03 & 0.06 & 0.6 & $-0.15-0.08$ & & 0.90 \\
fox ${ }^{*} \mathrm{~EB}$ & -0.31 & 0.13 & 2.4 & $-0.57--0.06$ & $\bullet$ & 0.88 \\
fox ${ }^{*} \mathrm{VH}$ & 0.47 & 0.16 & 2.9 & $0.15-0.78$ & $\bullet \bullet$ & 0.88 \\
fox ${ }^{*} \mathrm{QL2}$ & -0.28 & 0.09 & 3.3 & $-0.44--0.11$ & $\bullet \bullet$ & 0.90 \\
area size & 1.73 & 0.44 & 4.0 & $0.88-2.59$ & $\bullet \bullet \bullet$ & 1.00 \\
\hline
\end{tabular}

${ }^{\mathrm{A}}$ variables: $\mathrm{EB}=$ edible biomass $\left(\mathrm{g} \cdot \mathrm{m}^{-2}\right) ; \mathrm{VH}=$ vegetation height $(\mathrm{cm})$; QL2 $=-1 * 2^{\text {nd }}$ PCA component of forage quality: $\mathrm{NDF}(-)$ and $\mathrm{Ca}(+)$; fox $=$ red fox activity (log); area size $=$ area size of vegetation types $(\log ) ;{ }^{B}$ Beta's standardized by $2 *$ sd (Gelman, 2008). Beta of interaction is difference in slope between the two values when the co-variate increases 1 standard deviation; ${ }^{\mathrm{C}}$ Effect $=95 \%$ confidence interval does not include zero. $\bullet \mathrm{p}<0.05, \bullet \bullet \mathrm{p}<0.01, \bullet \bullet \bullet \mathrm{p}<0.001$. Models are based on: 979 observations of 11 hare in 20 vegetation types over 71 days; ${ }^{\mathrm{D}} \mathrm{Wp}$ : Akaike predictor weight.

The proportion of time hares spent foraging in a vegetation type was best explained by the model that included the interaction between fox activity and forage quality ( ${ }^{\text {nd }}$ PCA component) (Appendix 4, table A4.3). The top model was closely followed by a similar model that contained rabbit activity instead of fox activity. The top two models had a total relative weight of $91 \%$, and thus had the best fit to our data. Models that included the first PCA component of forage quality $(\mathrm{N}$ and $\mathrm{P})$ received lower relative model weights $(\leq 0.01)$ in the model set. 
Vegetation height and forage quality ( $2^{\text {nd }}$ PCA component) were on average negatively correlated with the proportion of time spent foraging, however, fox activity was positively correlated with the proportion of time spent foraging (table 4.3). Hare thus spent a higher proportion of time foraging in short vegetation types, and in vegetation types with a lower percentage of $\mathrm{Ca}$ and a higher percentage of NDF. They also spent a higher proportion of time foraging during days that foxes were more active. In tall vegetation, forage quantity (edible biomass) was negatively related to the proportion of time spent foraging (figure 4.2a), whereas in short vegetation, forage quantity was positively related to the proportion of time spent foraging (figure 4.2a). In vegetation types with more edible biomass, forage quality ( $2^{\text {nd }}$ PCA component) was less negatively related to the proportion of time spent foraging by hares (figure 4.2b). The effect of rabbit activity on the proportion of time spent foraging in a vegetation type was negatively related to vegetation height (figure $4.2 \mathrm{c})$ and forage quality ( $2^{\text {nd }}$ PCA component) (figure 4.2d). During days that rabbits were more active, hares thus spent a higher proportion of time foraging in short vegetation types, and in vegetation types that contained a relatively low quality of forage. Additionally, males spent a lower proportion of time foraging than females. The standardised coefficients show that forage quality, quantity, and vegetation height more strongly affected the proportion of time hares spent foraging in a vegetation type than the activity of foxes or rabbits. 
(A)

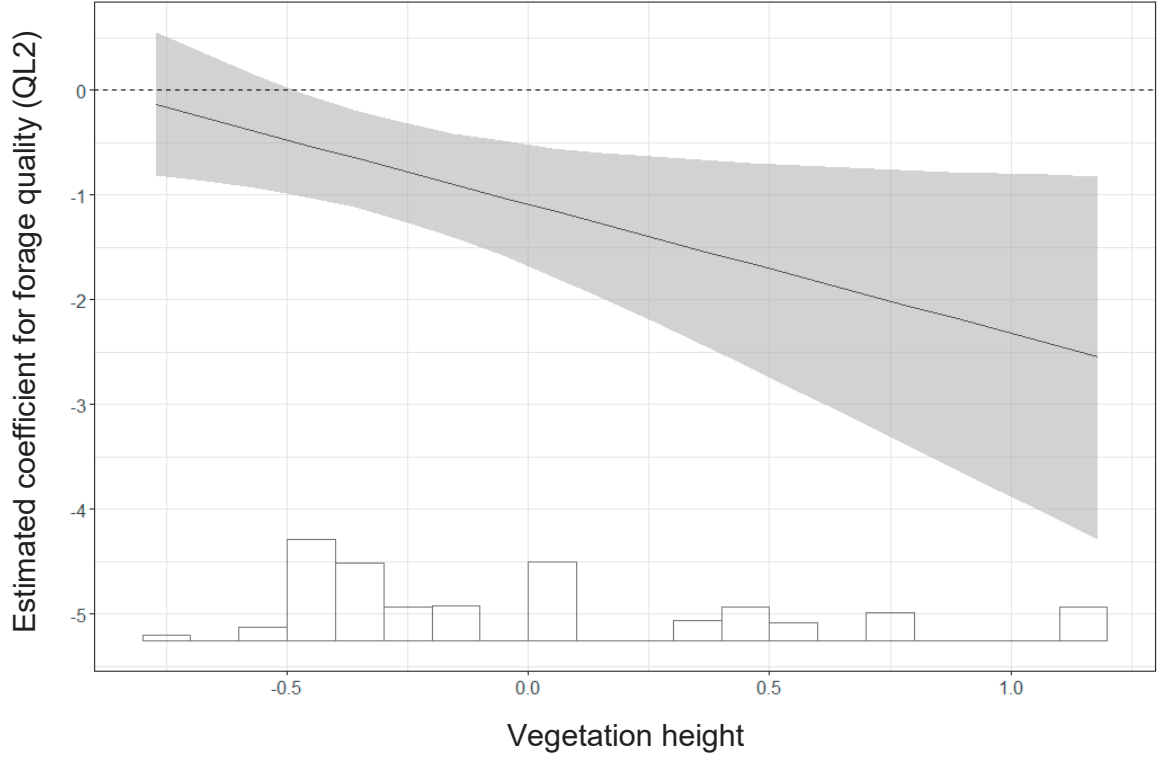

(B)

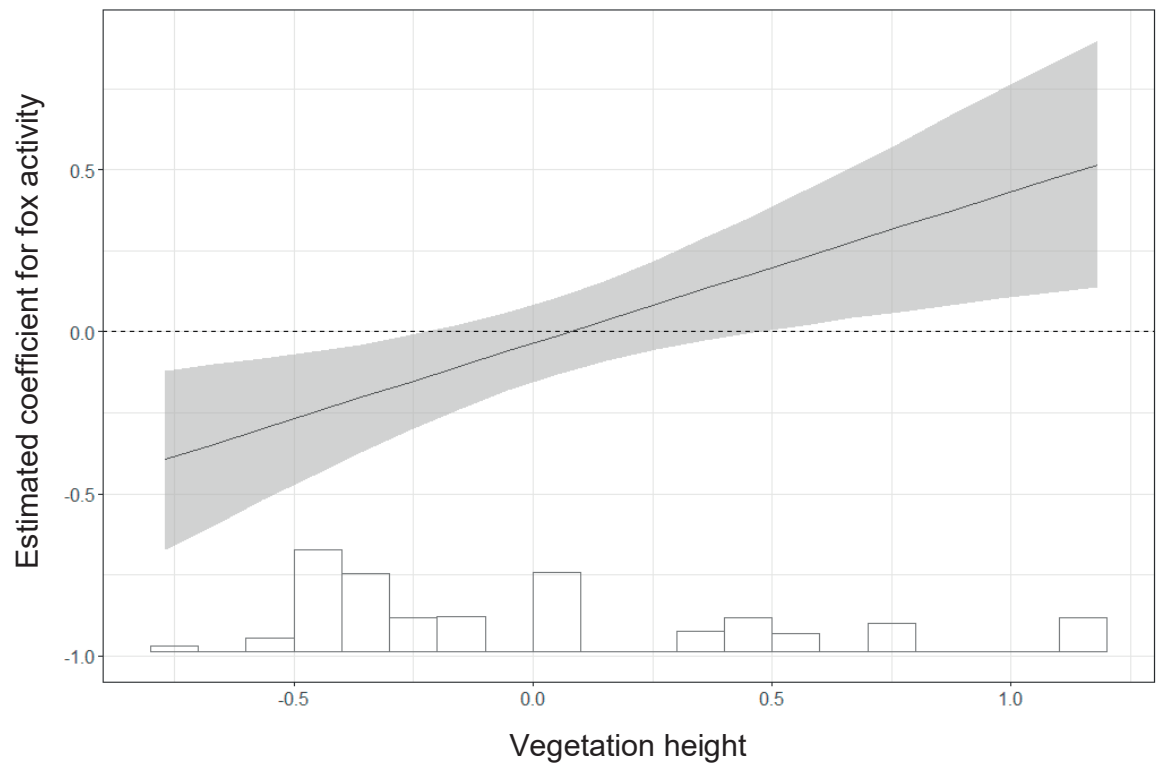

Figure 4.1: The estimated beta $(\beta)$ coefficient $(\bar{X} \pm 95 \% C I)$ between the proportion of GPS fixes of European hares in a vegetation type and (A) forage quality (NDF(-) and $\mathrm{Ca}(+))$ by vegetation height $(\mathrm{cm})$, (B) fox activity by vegetation height $(\mathrm{cm})$.

Histogram shows distribution of the conditional coefficient. 
(C)

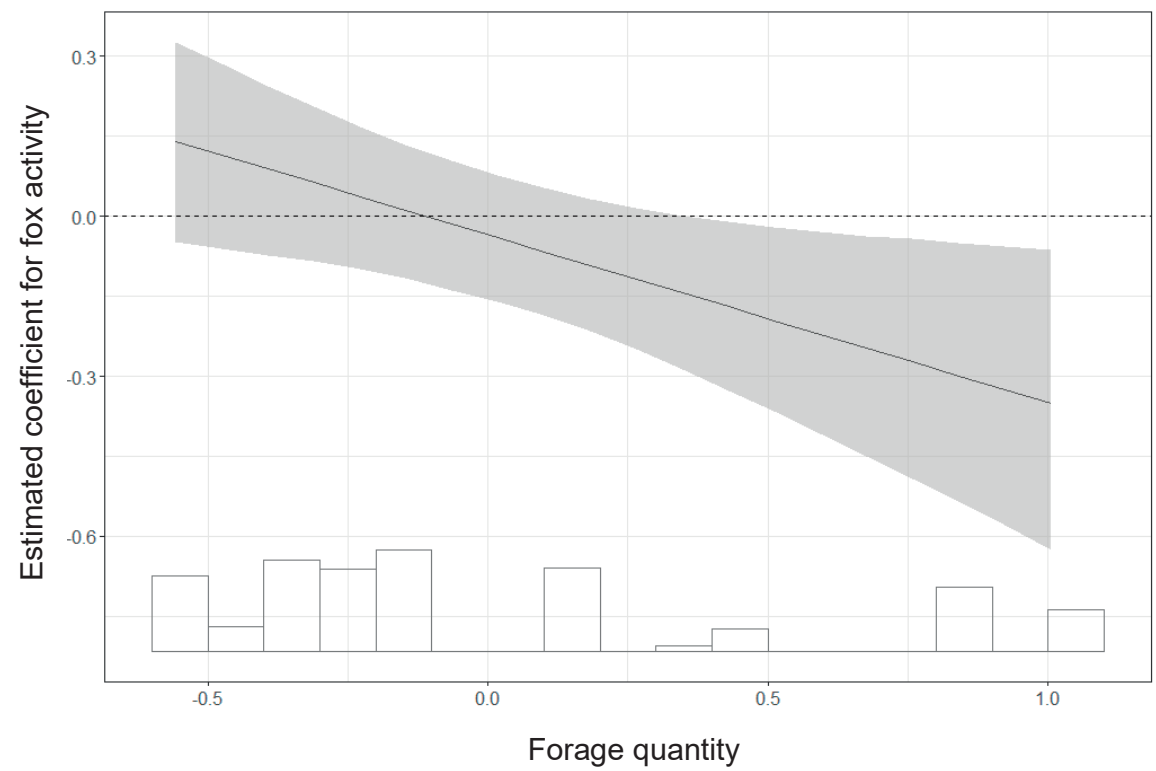

(D)

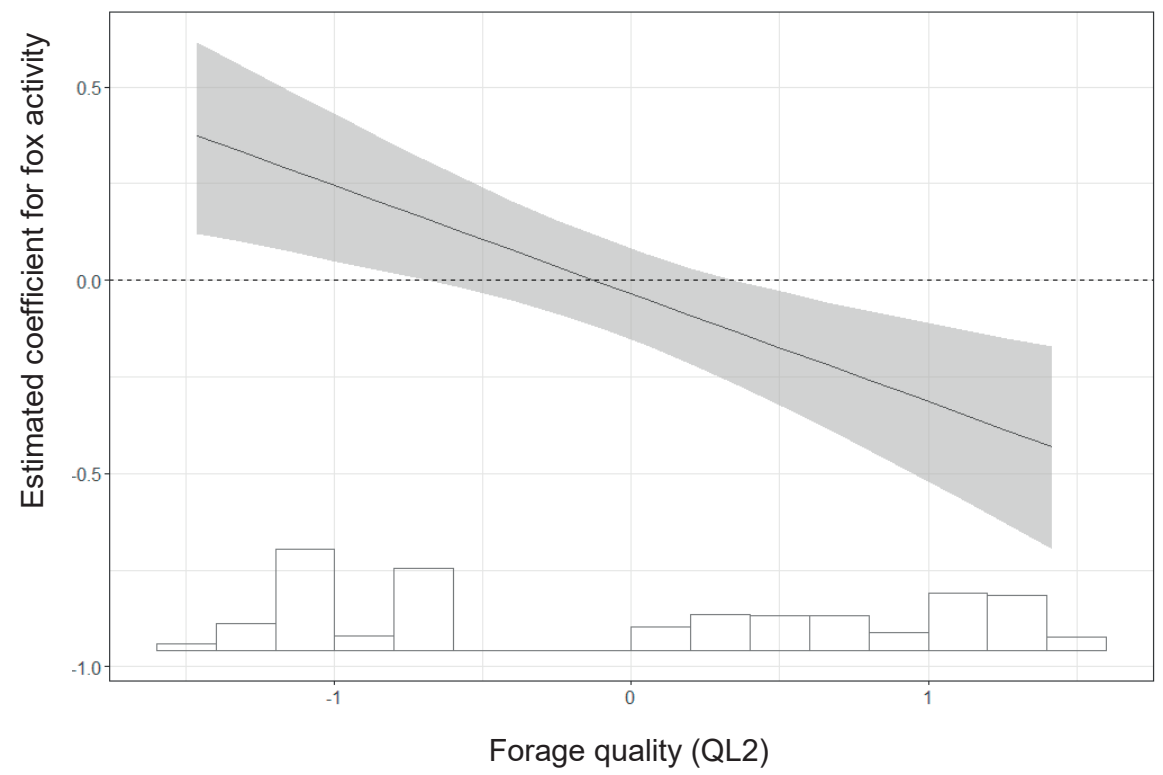

Figure 4.1: The estimated beta $(\beta)$ coefficient $(\bar{X} \pm 95 \% C I)$ between the proportion of GPS fixes of European hares in a vegetation type and (C) fox activity by forage quantity (the amount of edible biomass), and (D) fox activity by forage quality (NDF(-) and $\mathrm{Ca}(+))$.

Histogram shows distribution of the conditional coefficient. (continued) 
Table 4.3: Results of full-model conditional averaging of all parsimonious generalized linear mixed models on the effect of predator and competitor activity and its interaction with forage quality, quantity and vegetation height on the proportion of time spent foraging of European hares in a vegetation type.

\begin{tabular}{|c|c|c|c|c|c|c|}
\hline Variables ${ }^{\mathrm{A}}$ & $\begin{array}{l}\text { Estimate } \\
(\beta)^{B}\end{array}$ & $\frac{\text { (conditional) }}{\left(\mathrm{SE}_{\beta}\right)}$ & $Z$ value & $2.5 \%-97.5 \%$ C.I. & Effect ${ }^{\mathrm{C}}$ & $\mathrm{W}_{\mathrm{p}}{ }^{\mathrm{D}}$ \\
\hline Intercept & -0.71 & 0.13 & 5.3 & $-0.97--0.45$ & $\bullet \bullet \bullet$ & 1.00 \\
\hline $\mathrm{EB}$ & 0.09 & 0.19 & 0.5 & $-0.28-0.46$ & & 0.05 \\
\hline VH & -0.58 & 0.20 & 2.9 & $-0.97--0.19$ & $\bullet \bullet$ & 0.01 \\
\hline $\mathrm{EB}^{*} \mathrm{VH}$ & -1.14 & 0.57 & 2.0 & $-2.25--0.02$ & $\bullet$ & $<0.01$ \\
\hline QL1 & 0.19 & 0.13 & 1.5 & $-0.06-0.45$ & & $<0.01$ \\
\hline QL2 & -0.43 & 0.10 & 4.3 & $-0.62--0.23$ & $\bullet \bullet \bullet$ & 0.99 \\
\hline QL2*EB & 0.46 & 0.20 & 2.3 & $0.07-0.85$ & $\bullet$ & 0.05 \\
\hline Fox & 0.14 & 0.05 & 2.7 & $0.04-0.23$ & $\bullet \bullet$ & 0.65 \\
\hline fox ${ }^{*} V H$ & -0.09 & 0.07 & 1.2 & $-0.24-0.06$ & & $<0.01$ \\
\hline fox ${ }^{*} \mathrm{QL1}$ & -0.09 & 0.06 & 1.6 & $-0.21-0.02$ & & $<0.01$ \\
\hline fox*QL2 & -0.06 & 0.04 & 1.4 & $-0.14-0.02$ & & 0.64 \\
\hline Rabbit & 0.05 & 0.05 & 0.9 & $-0.06-0.15$ & & 0.27 \\
\hline rabbit*VH & -0.19 & 0.08 & 2.5 & $-0.34--0.04$ & $\bullet$ & $<0.01$ \\
\hline rabbit*QL2 & -0.12 & 0.04 & 2.7 & $-0.20--0.03$ & $\bullet \bullet$ & 0.27 \\
\hline $\operatorname{sex}^{\mathrm{E}}$ & -0.48 & 0.20 & 2.4 & $-0.87-0.09$ & $\bullet$ & 1.00 \\
\hline $\operatorname{area~type~}^{\mathrm{F}}$ & -0.25 & 0.19 & 1.3 & $-0.63-0.13$ & & 1.00 \\
\hline
\end{tabular}

${ }^{\mathrm{A}}$ variables: $\mathrm{EB}=$ edible biomass $\left(\mathrm{g} \cdot \mathrm{m}^{-2}\right) ; \mathrm{VH}=$ vegetation height $(\mathrm{cm}) ; \mathrm{QL1}=1^{\text {st }}$ PCA component of forage quality: $\mathrm{N}$ and P; QL2 $=-1 * 2^{\text {nd }}$ PCA component of forage quality: NDF (-) and Ca (+); fox $=$ red fox activity (log); rabbit $=$ rabbit activity; ${ }^{\mathrm{B}}$ Beta's standardized by $2^{*} \mathrm{sd}$ (Gelman, 2008). Beta of interaction is difference in slope between the two values when the co-variate increases 1 standard deviation; ${ }^{\mathrm{C}}$ Effect $=95 \%$ confidence interval does not include zero. $\circ \mathrm{p}<0.1, \bullet \mathrm{p}<0.05, \bullet \bullet \mathrm{p}<$ $0.01, \bullet \bullet \bullet p<0.001$. Models are based on: 2843 observations of 11 hare in 19 vegetation types in 2 areas over 79 days; ${ }^{\mathrm{D}} \mathrm{Wp}$ : Akaike predictor weight; ${ }^{\mathrm{E}}$ reference category for Sex is female; ${ }^{\mathrm{F}}$ reference category for Area type is Vennewater. 
(A)

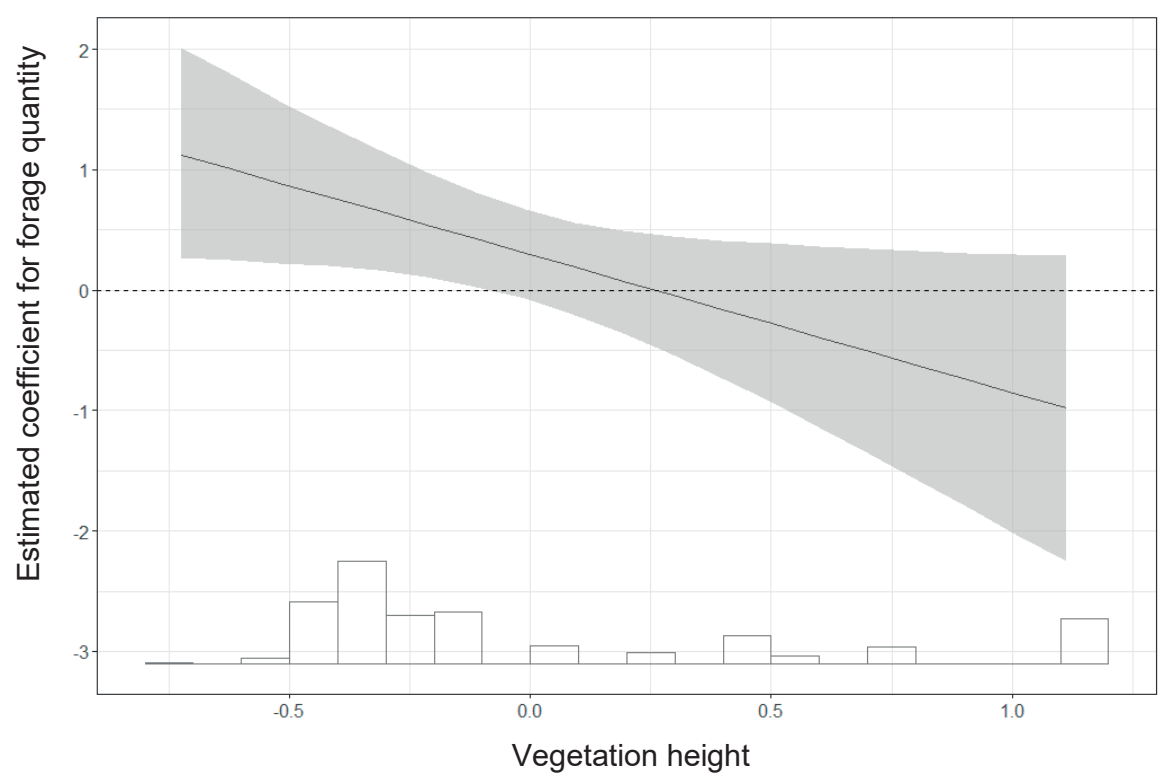

(B)

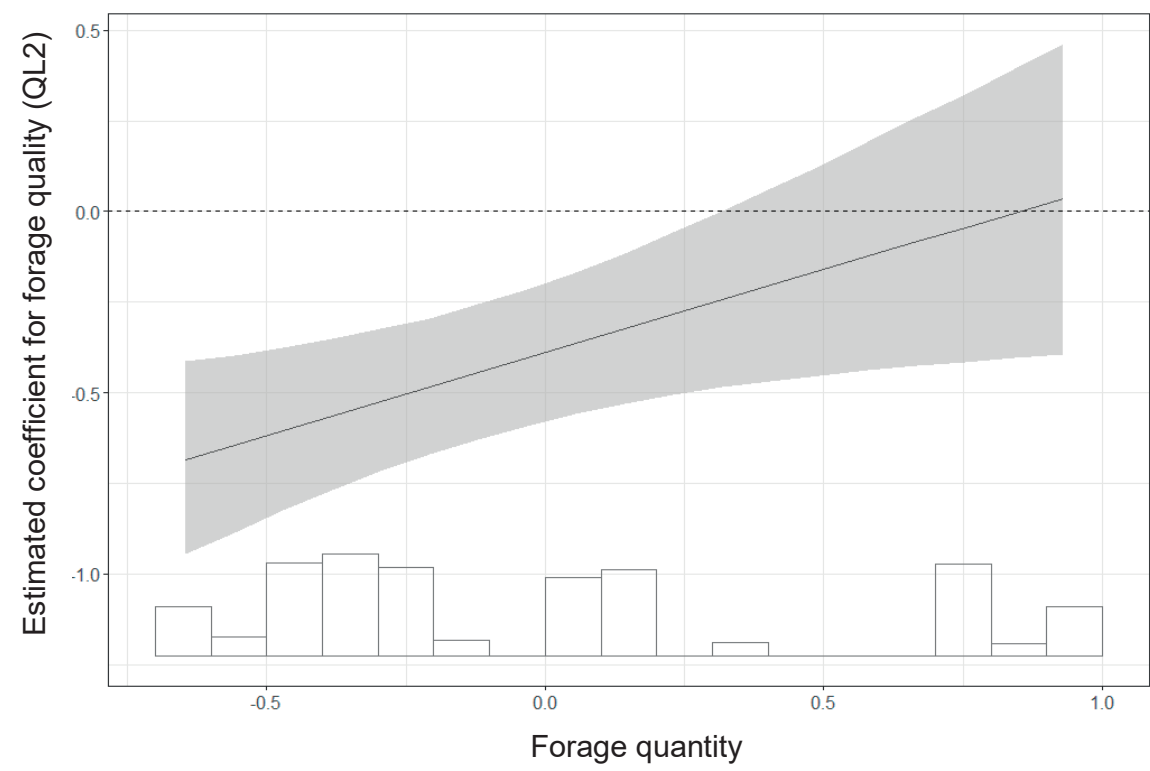

Figure 4.2: The estimated beta $(\beta)$ coefficient $(\bar{X} \pm 95 \% C I)$ between the proportion of time spent foraging by European hares in a vegetation type and $(A)$ forage quantity (the amount of edible biomass) by vegetation height (cm), (B) forage quality (NDF(-) and $\mathrm{Ca}(+)$ ) by forage quantity (the amount of edible biomass), (C) rabbit activity by vegetation height (cm), (D) rabbit activity by forage quality (NDF(-) and $\mathrm{Ca}(+))$.

Histogram shows distribution of the conditional coefficient. 
(C)

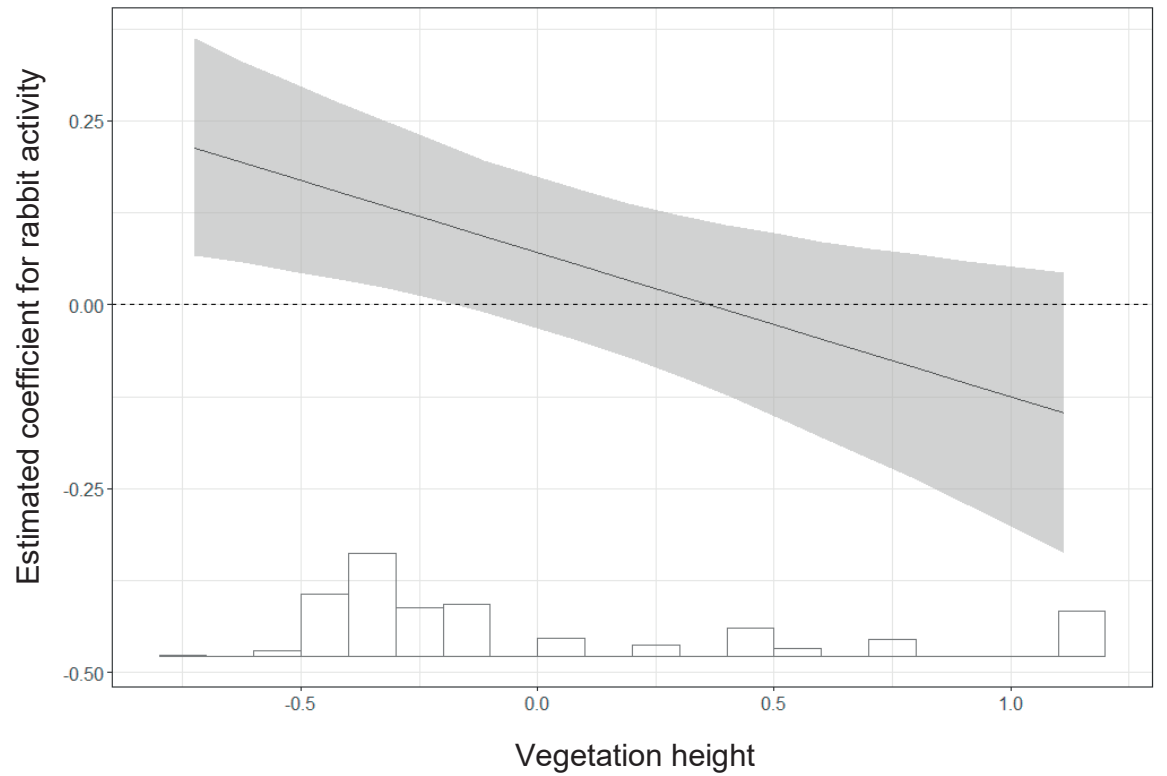

(D)

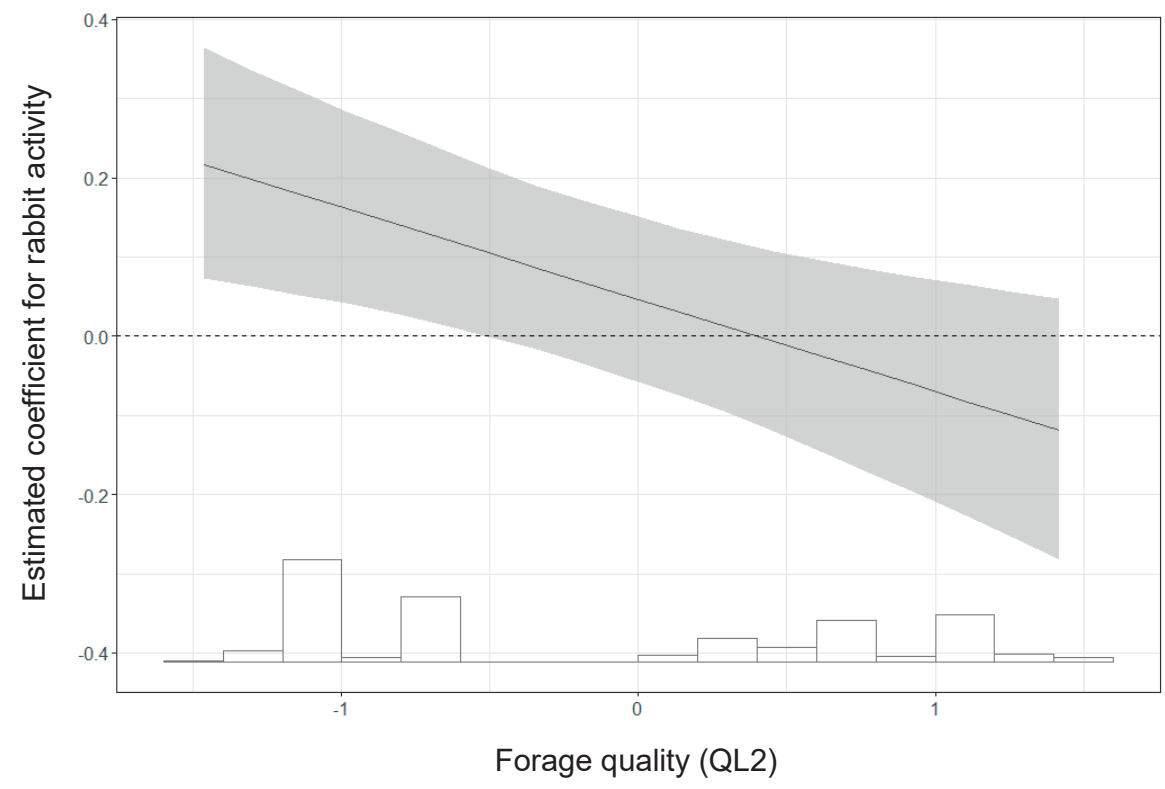




\section{DISCUSSION}

We have tested the relative importance of predator and competitor activity, and forage quality and quantity for the proportion of time spent in a vegetation type and the proportion of time spent foraging by the intermediate-sized herbivore European hare. The relative importance in determining where and what intermediate-sized herbivores forage still remains unclear (Arsenault \& Owen-Smith, 2002; Brown \& Kotler, 2004; Morris, 2009). Whereas most studies (> 75\%) that investigate the trade-off between foraging behaviour and predation risk using giving-up density focus on small rodents $(<1 \mathrm{~kg})$ (Verdolin, 2006) (e.g., squirrels, mice and voles), very few studies focus on intermediate-sized $(<20 \mathrm{~kg})$ herbivores (but see e.g., Hodges \& Sinclair, 2005; Shrader et al., 2012; Crowell et al., 2016) and include the effects of competition. Small rodents are central-place foragers that can use their burrow as a refuge. Unlike central-place foragers, hares are free ranging and use a different foraging strategy, as well as a different response towards predators and competitors (Potts et al., 2012; Shrader et al., 2012). Besides, studies that focus on giving-up density are limited by the artificiality of the food patches, especially the quality of the food offered, and the predictability of the food patch (Bedoya-Perez et al., 2013). Nevertheless, predator or competitor behaviour that can limit nutrient intake through changes in foraging behaviour is very important for small and intermediate-sized herbivores, because nutritional requirements increase with declining body mass (Demment \& Van Soest, 1985).

Our first expectation was that when predators and smaller competitors were more active, intermediate-sized herbivores spent more time in vegetation types that contained a lower food quality. We found that increased activity by smaller competitors did not affect the proportion of time hares spent in a certain vegetation type. However, during increased activity of predators, hares spent a higher proportion of time in low-risk vegetation types that had tall vegetation, or a low food quality or quantity. In this study, food quality and vegetation height were measured separately and were not ( $\mathrm{N}$ and $\mathrm{P}$ concentration) too moderately (Ca and NDF concentration) correlated with vegetation height. Therefore, we interpreted vegetation height as an indicator for prey cover only. Tall structure rich vegetation provides cover and protection for prey against predators (Verdolin, 2006), and is used as resting place by hares during the day (Neumann et al., 2011). Besides, hares make use of cryptic coloration in tall vegetation to evade predators (Focardi \& Rizzotto, 1999). Unlike European hares, snowshoe hares (Hodges \& Sinclair, 2005) and roe deer (Samelius et al., 2013) did not spent more time in low-risk vegetation types to reduce predation risk, possibly because of differences 
in predator type or prey escape mode (Wirsing et al., 2010). Even though hares use flight in short vegetation to escape predators (Focardi \& Rizzotto, 1999), we found that high fox activity negatively affected the proportion of time that hares spent in short vegetation. The reason that hare does not spend more time in short vegetation during times of high risk is probably that hares cannot detect foxes early enough or escape from these foxes if patches of short vegetation are smaller than their minimum flight distance. Prey escape mode (Wirsing et al., 2010) and landscape features (Heithaus et al., 2009), such as the small size of patches or patch distribution, may thus favour fox hunting in patches of short vegetation (Kauffman et al., 2007; Weterings et al., 2016). It seems that habitat shifts as a result of the antipredator behaviour of hare is context dependent (Kuijper et al., 2015), namely that it depends on the patch size of the vegetation.

Independent of predator activity, hares also spent more time in vegetation types that contained a low food quality. Especially in winter, hare can forage on grasses that contain a higher concentration of fibres with lower levels of lignin compared to dicotyledonous species (Iason \& Van Wieren, 1999).

Our second expectation was that if intermediate-sized herbivores had to spend more time in vegetation types that contained a lower food quality they also had to spend more time on foraging. As expected, our hares spent a higher proportion of time spent foraging in vegetation types with a higher concentration of fibres (i.e., NDF) in the edible biomass, especially in vegetation types with a low edible biomass. As plant bite sizes are correlated to biomass, smaller bites in vegetation types with a lower edible biomass require more handling time and will thus reduce forage intake (Shipley, 2007) and increase foraging time (Heuermann et al., 2011). Additionally, the concentration of fibres in the food will negatively affect forage intake, although this strongly depends on the type of herbivore digestion system (Bell, 1971). In vegetation types with a higher concentration of fibres, hares, which have a relatively short digestion system, maximize the passage rate of forage (Stott, 2007), and thus spent more time to foraging. Remarkably, the proportion of time spent foraging in a vegetation type not only increased in low-risk, low-quality vegetation types, it also increased when foxes were more active. This implies that hares not only perceived a predation risk that was nonuniformly spread over the landscape (i.e., low and high risk vegetation types) (Kotler \& Blaustein, 1995), but hares also perceived a predation risk that was uniformly spread over the landscape. Prey increase their time spent foraging if they have no safe refuges from predators (i.e., free-ranging herbivores), especially 
if "predator and prey are of similar body size and locomotion" (Eccard et al., 2008, p.726), like the European hare and the red fox.

Besides that hare foraging behaviour was affected by fox activity, hares spent a higher proportion of time foraging when rabbits were more active, especially in short vegetation types and vegetation types with a high concentration of fibres. First, spending time in vegetation types with a high concentration of fibres would allow the larger hare to avoid competition with the smaller rabbit (given that larger herbivores have the ability to tolerate low forage quality; Bell, 1971), but increased the proportion of foraging time. Second, rabbits can attract predators (Smith \& Quin, 1996; Norbury, 2001), and because of this, hares should allocate more time to feeding in low-risk locations (Lima \& Bednekoff, 1999, Verdolin, 2006). It implicates that rabbits dilute predation risk for hares in the risky open vegetation types, particularly, because rabbits are the stronger competitor (Flux, 2008), and because rabbits experience a higher individual mortality risk by predation than hares (Grand \& Dill, 1999b). This would mean that when rabbits are active, hare would select plants with a higher fibre concentration in open vegetation types, while rabbits would select plants with a lower fibre concentration in open vegetation types.

Our results show that food quality and quantity more strongly affected hare foraging behaviour than the activity of predators, whereas the activity of smaller competitors was least important. Predation might be less strong than the effect of resource acquisition, probably because the relative size difference between our prey species and its predator was small (Sinclair et al., 2003). The range in nutrient concentrations measured in the edible biomass (Appendix 4, table A4.1) seems to reflect the natural variability in coastal-dune landscapes (e.g., see \% of NDF in Lamoot, 2004). The absence of intraspecific-group competition (Grand \& Dill, 1999a), and the low density of smaller competitors (Hopcraft et al., 2010) in the coastal-dune landscape, possibly marginalised the effects of small competitor activity on hare foraging time. Additionally, predation is stronger than competition in the landscapes of high resource availability (Chesson \& Kuang, 2008) that are present in the Dutch dune-coastal landscape (Kooijman et al., 1998), were Calcium is not a limiting resource (Barboza et al., 2009).

Few studies simultaneously consider the trade-off between resource acquisition, predation and competition, especially for free-ranging herbivores of intermediatesize, whereas this trade-off has been studied for smaller-sized (e.g., Halliday \& Morris, 2013) and larger-sized (e.g., Vijayan et al., 2012) herbivores in more detail. 
By investigating the relative importance of these factors that affect behavioural trade-offs in complex landscapes, we can get insight into the mechanisms that determine spatial distribution of herbivores. Although small and intermediate-sized herbivore behaviour or habitat use is predicted to be controlled by predation risk (i.e., landscape of fear), our study shows that food quality and quantity is more important. It seems that we need to reappraise the importance of the landscape of food in a world of fear. 


\section{APPENDIX 4}

Table A4.1: Characteristics of the vegetation types in the coastal dune landscape 'Noordhollands Duinreservaat' near Castricum, the Netherlands.

\begin{tabular}{|c|c|c|c|c|c|}
\hline Vegetation type & $\begin{array}{c}\text { Area } \\
\text { size (ha) }\end{array}$ & $\begin{array}{l}\text { \# hares } \\
\text { foraging }\end{array}$ & $\begin{array}{l}\text { \# camera } \\
\text { locations }\end{array}$ & \# plots & $\begin{array}{c}\text { Average } \\
\text { percentage of } \\
\text { GPS fixes/day } \\
\text { of hares }( \pm S D)\end{array}$ \\
\hline Agriculture & 40.8 & 10 & - & 6 & $21.3 \pm 9.7$ \\
\hline Flower rich grasslands & 43.3 & 6 & 24 & 5 & $9.0 \pm 7.4$ \\
\hline Bulb fields & 1.3 & 2 & - & 1 & $1.2 \pm 1.5$ \\
\hline Dune grasslands & 74.0 & 11 & 59 & 6 & $11.8 \pm 6.4$ \\
\hline $\begin{array}{l}\text { Burnet rose, creeping willow-, } \\
\text { blackberry thicket }\end{array}$ & 37.0 & 8 & 29 & 6 & $5.9 \pm 4.9$ \\
\hline Bare sand & 3.6 & 5 & - & 7 & $0.8 \pm 0.7$ \\
\hline Calcareous dune grassland & 125.2 & 11 & 74 & 5 & $14.7 \pm 7.9$ \\
\hline Calcareous dune valleys & 2.9 & 1 & 2 & 5 & $1.0 \pm 1.5$ \\
\hline Deciduous forest & 65.1 & 8 & 4 & 3 & $2.3 \pm 2.8$ \\
\hline Coniferous forest & 37.0 & 6 & - & 1 & $0.5 \pm 1.2$ \\
\hline Former agriculture & 7.5 & 2 & - & 3 & $7.2 \pm 3.4$ \\
\hline Other & 6.7 & 3 & - & 2 & $0.8 \pm 1.2$ \\
\hline Other forests & 18.5 & 7 & 1 & 4 & $2.5 \pm 2.1$ \\
\hline Reed swamp & 17.4 & 3 & 1 & 2 & $4.3 \pm 6.6$ \\
\hline Reed swamp communities & 0.7 & 4 & 1 & 2 & $0.5 \pm 0.7$ \\
\hline $\begin{array}{l}\text { Herbaceous, fault and mantle } \\
\text { communities }\end{array}$ & 3.7 & 8 & - & 4 & $1.1 \pm 1.5$ \\
\hline Thickets & 75.6 & 11 & 6 & 4 & $8.2 \pm 4.4$ \\
\hline Nutrient rich grasslands & 20.8 & 9 & 2 & 5 & $8.1 \pm 7.4$ \\
\hline $\begin{array}{l}\text { Nutrient-rich pioneer communities, } \\
\text { flood meadows and pace vegetation }\end{array}$ & 1.9 & 4 & 1 & 6 & $1.8 \pm 2.5$ \\
\hline Near-shore communities & 13.5 & 3 & 4 & 6 & $0.7 \pm 0.9$ \\
\hline
\end{tabular}


Nutrients in the vegetation Forage

$(\%)$

quality

\begin{tabular}{|c|c|c|c|c|c|c|c|c|c|}
\hline $\begin{array}{l}\text { Plant species } \\
\text { in hare diet } \dagger\end{array}$ & $\begin{array}{c}\text { Average } \\
\text { percentage } \\
\text { of time spent } \\
\text { foraging }( \pm S D)\end{array}$ & $\begin{array}{l}\text { Biomass } \\
\left(\mathrm{g} \cdot \mathrm{m}^{-2}\right)^{*}\end{array}$ & $\begin{array}{c}\text { Edible } \\
\text { biomass } \\
\left(\mathrm{g} \cdot \mathrm{m}^{-2}\right) \ddagger\end{array}$ & $\mathrm{N}$ & $\mathrm{P}$ & $\mathrm{Ca}$ & NDF & QL1 & QL2 \\
\hline $2,3,4,5,6$ & $56.0 \pm 12.3$ & 105 & 40 & 3.7 & 0.4 & 0.5 & 48 & 0.2 & 0.6 \\
\hline $1,2,4,6$ & $43.1 \pm 22.0$ & 148 & 58 & 3.6 & 0.4 & 0.5 & 51 & -0.2 & 1.1 \\
\hline 8 & $37.3 \pm 19.3$ & 642 & 210 & 5.1 & 0.7 & 0.3 & 39 & 3.3 & -0.9 \\
\hline $1,2,3,5,6,7$ & $41.3 \pm 23.2$ & 242 & 110 & 3.6 & 0.3 & 1.1 & 41 & 0.0 & -0.6 \\
\hline $1,2,3,4,6,7$ & $30.8 \pm 23.9$ & 418 & 227 & 4.0 & 0.4 & 0.9 & 43 & 0.5 & -0.2 \\
\hline $1,2,3,7$ & $36.2 \pm 16.2$ & 12 & 6 & 3.7 & 0.3 & 0.6 & 48 & -0.1 & 0.7 \\
\hline $1,2,3,4,7$ & $36.2 \pm 24.2$ & 467 & 207 & 3.3 & 0.3 & 0.5 & 51 & -0.6 & 1.2 \\
\hline $1,2,3,4,5,6$ & $57.4 \pm 32.7$ & 243 & 138 & 4.1 & 0.4 & 0.9 & 42 & 0.7 & -0.3 \\
\hline $2,4,5,7$ & $29.4 \pm 30.5$ & 10 & 4 & 4.1 & 0.5 & 0.6 & 45 & 1.1 & -0.1 \\
\hline 7 & $20.3 \pm 15.2$ & 2 & 0 & 2.6 & 0.2 & 1.6 & 35 & -1.3 & -1.5 \\
\hline $1,2,4,7$ & $22.2 \pm 15.1$ & 153 & 65 & 3.7 & 0.4 & 0.5 & 48 & 0.2 & 0.7 \\
\hline 2 & $45.5 \pm 28.6$ & 1 & 0 & 3.6 & 0.3 & 0.4 & 53 & -0.4 & 1.4 \\
\hline $2,3,5,7$ & $32.3 \pm 24.3$ & 256 & 52 & 2.9 & 0.3 & 1.2 & 41 & -1.0 & -0.5 \\
\hline $4,5,7$ & $52.1 \pm 27.5$ & 429 & 39 & 2.8 & 0.2 & 1.5 & 36 & -1.1 & -1.3 \\
\hline $1,2,3,4,5,6$ & $33.0 \pm 25.4$ & 79 & 31 & 3.7 & 0.4 & 0.5 & 49 & 0.2 & 0.8 \\
\hline $2,4,5,7$ & $35.3 \pm 26.0$ & 81 & 9 & 2.9 & 0.3 & 1.4 & 38 & -0.8 & -1.2 \\
\hline $1,2,4,5,7$ & $27.9 \pm 24.3$ & 360 & 97 & 3.1 & 0.3 & 1.4 & 37 & -0.7 & -1.1 \\
\hline $1,2,3,4,5,6$ & $49.0 \pm 24.1$ & 88 & 32 & 3.5 & 0.4 & 0.4 & 51 & -0.1 & 1.1 \\
\hline $2,3,4,5$ & $31.9 \pm 23.1$ & 67 & 25 & 3.6 & 0.4 & 0.4 & 53 & -0.3 & 1.3 \\
\hline $1,2,3,4,7$ & $30.1 \pm 22.5$ & 267 & 145 & 4.1 & 0.3 & 1.2 & 37 & 0.7 & -1.1 \\
\hline
\end{tabular}

$\mathrm{NDF}=$ neutral detergent fibre on ash-in-basis; $\mathrm{QL1}=1^{\text {st }} \mathrm{PCA}$ component nutrient quality of the vegetation; QL2 $=2^{\text {nd }}$ PCA component nutrient quality of the vegetation. ${ }^{\dagger}$ Plant species are ordered by fibre concentration from high to low: $1=$ Festuca rubra, $2=$ Agrostis capillaris, $3=$ Poa pratensis, $4=$ Holcus lanatus, $5=$ Poa trivialis, $6=$ Taraxacum officinale, $7=$ Rubus caesisus, $8=$ commercial bulb species; ${ }^{\ddagger}$ (edible) biomass is calculated up to a height of $50 \mathrm{~cm}$. 
Table A4.2: Results of the generalized linear mixed model on the effect of predator and competitor activity and its interaction with forage quality, quantity and vegetation height on the proportion of GPS fixes of European hares in a vegetation type.

\begin{tabular}{|c|c|c|c|c|c|}
\hline Rank & Model type & $D f$ & AICc & $\triangle \mathrm{AIC}$ & $w_{i} \dagger$ \\
\hline 1 & $\mathrm{~EB}+\mathrm{VH}+\mathrm{QL} 2+$ fox + fox* $\mathrm{EB}+$ fox* $\mathrm{VH}+$ fox* $\mathrm{QL} 2$ & 14 & 8672.4 & 0.0 & 0.87 \\
\hline 2 & $\mathrm{VH}+\mathrm{QL} 2+\mathrm{QL} 2 * \mathrm{VH}$ & 10 & 8677.2 & 4.8 & 0.08 \\
\hline 3 & $\mathrm{QL} 2+$ fox + fox* QL2 & 10 & 8679.6 & 7.3 & 0.02 \\
\hline \multirow[t]{3}{*}{4} & QL2 & 8 & 8680.4 & 8.0 & 0.02 \\
\hline & $\begin{array}{l}\mathrm{EB}+\mathrm{VH}+\mathrm{QL} 2+\text { rabbit + rabbit }{ }^{*} \mathrm{~EB}+\text { rabbit }^{*} \mathrm{VH}+ \\
\text { rabbit }{ }^{*} \mathrm{QL} 2\end{array}$ & 14 & 8681.3 & 8.9 & \\
\hline & $\mathrm{EB}+\mathrm{QL} 2+\mathrm{QL} 2 * \mathrm{~EB}$ & 10 & 8681.7 & 9.4 & \\
\hline \multirow[t]{18}{*}{5} & Intercept & 7 & 8681.7 & 9.4 & $<0.01$ \\
\hline & QL2 + rabbit + rabbit*QL2 & 10 & 8681.8 & 9.4 & \\
\hline & EB & 8 & 8682.3 & 10.0 & \\
\hline & QL1 & 8 & 8682.8 & 10.4 & \\
\hline & $E B+$ fox + fox ${ }^{*} E B$ & 10 & 8683.0 & 10.7 & \\
\hline & $\mathrm{EB}+\mathrm{VH}+\mathrm{EB}^{*} \mathrm{VH}$ & 10 & 8683.1 & 10.7 & \\
\hline & $\mathrm{VH}$ & 8 & 8683.5 & 11.1 & \\
\hline & Fox & 8 & 8683.7 & 11.3 & \\
\hline & Rabbit & 8 & 8683.7 & 11.4 & \\
\hline & $\mathrm{QL1}+$ fox + fox* QL1 & 10 & 8684.1 & 11.7 & \\
\hline & $\mathrm{EB}+\mathrm{QL} 1+\mathrm{QL} 1 * \mathrm{~EB}$ & 10 & 8685.7 & 13.3 & \\
\hline & $\mathrm{VH}+\mathrm{QL1}+\mathrm{QL1}{ }^{*} \mathrm{VH}$ & 10 & 8686.0 & 13.7 & \\
\hline & $\mathrm{EB}+$ rabbit + rabbit*EB & 10 & 8686.2 & 13.9 & \\
\hline & $\mathrm{QL} 1$ + rabbit + rabbit*QL1 & 10 & 8686.4 & 14.0 & \\
\hline & $V H+$ fox + fox*VH & 10 & 8687.4 & 15.0 & \\
\hline & $\mathrm{VH}+$ rabbit + rabbit $^{*} \mathrm{VH}$ & 10 & 8687.4 & 15.1 & \\
\hline & $\mathrm{EB}+\mathrm{VH}+\mathrm{QL} 1+$ fox + fox*EB + fox* ${ }^{*} \mathrm{H}+$ fox* QL1 & 14 & 8689.4 & 17.0 & \\
\hline & $\begin{array}{l}\mathrm{EB}+\mathrm{VH}+\mathrm{QL} 1+\text { rabbit + rabbit*EB + rabbit*VH + } \\
\text { rabbit*QL1 }\end{array}$ & 14 & 8692.9 & 20.5 & \\
\hline
\end{tabular}

AICc $=$ Aikaike information criterion corrected for small sample size; $\triangle \mathrm{AICc}=$ delta AICc with regard to best fitting model; $\mathrm{w}_{\mathrm{i}}=$ Akaike weight or relative weight of each model. $\dagger$ Only parsimonious models were weighted, i.e., more complex models with lower AICc (shaded) were left out. Model parameters: QL1 $=1^{\text {st }}$ PCA component of forage quality: $\mathrm{N}$ and P; QL2 $=2^{\text {nd }}$ PCA component of forage quality: $\mathrm{NDF}(+)$ and $\mathrm{Ca}(-) ; \mathrm{EB}=$ edible biomass $\left(\mathrm{g} \cdot \mathrm{m}^{-2}\right) ; \mathrm{VH}=$ vegetation height $(\mathrm{cm})$; fox = red fox activity; rabbit $=$ rabbit activity. All models contained the control variable area size. Models are based on: 979 observations of 11 hare in 20 vegetation types over 71 days. 
Table A4.3: Results of the generalized linear mixed model on the effect of predator and competitor activity and its interaction with forage quality and -quantity on the proportion of time spent foraging of European hares in a vegetation type.

\begin{tabular}{|c|c|c|c|c|c|}
\hline Rank & Model type & Df & AICc & $\triangle \mathrm{AIC}$ & $w_{i} \dagger$ \\
\hline 1 & $\mathrm{QL} 2+$ fox + fox* QL2 & 12 & 36771.8 & 0.0 & 0.64 \\
\hline 2 & $\mathrm{Q} L 2$ + rabbit + rabbit* QL2 & 12 & 36773.5 & 1.7 & 0.27 \\
\hline 3 & $\mathrm{~EB}+\mathrm{QL} 2+\mathrm{QL} 2 * \mathrm{~EB}$ & 12 & 36776.8 & 5.0 & 0.05 \\
\hline \multirow[t]{4}{*}{4} & QL2 & 10 & 36778.2 & 6.4 & 0.03 \\
\hline & $\mathrm{EB}+\mathrm{VH}+\mathrm{QL} 2+$ fox + fox ${ }^{*} \mathrm{~EB}+\mathrm{fox}^{*} \mathrm{VH}+\mathrm{fox}^{*} \mathrm{QL} 2$ & 16 & 36778.8 & 7.0 & \\
\hline & $\begin{array}{l}\mathrm{EB}+\mathrm{VH}+\mathrm{QL} 2+\text { rabbit + rabbit }{ }^{*} \mathrm{~EB}+\text { rabbit }^{*} \mathrm{VH}+ \\
\text { rabbit }{ }^{*} \mathrm{QL} 2\end{array}$ & 16 & 36779.1 & 7.3 & \\
\hline & $\mathrm{VH}+\mathrm{QL} 2+\mathrm{QL} 2 * \mathrm{VH}$ & 12 & 36781.4 & 9.6 & \\
\hline \multirow[t]{2}{*}{5} & $V H+$ fox + fox* VH & 12 & 36781.8 & 10.0 & $<0.01$ \\
\hline & $\mathrm{EB}+\mathrm{VH}+\mathrm{QL} 1+$ fox + fox ${ }^{*} \mathrm{~EB}+\mathrm{fox}^{*} \mathrm{VH}+\mathrm{fox}^{*} \mathrm{QL} 1$ & 16 & 36782.0 & 10.2 & \\
\hline 6 & $\mathrm{VH}+$ rabbit + rabbit $* \mathrm{VH}$ & 12 & 36783.8 & 12.0 & $<0.01$ \\
\hline 7 & $\mathrm{~EB}+\mathrm{VH}+\mathrm{EB}^{*} \mathrm{VH}$ & 12 & 36785.9 & 14.1 & $<0.01$ \\
\hline \multirow[t]{2}{*}{8} & $\mathrm{QL1}+$ fox + fox* QL1 & 12 & 36786.8 & 15.0 & $<0.01$ \\
\hline & $\begin{array}{l}\mathrm{EB}+\mathrm{VH}+\mathrm{QL} 1+\text { rabbit + rabbit }{ }^{*} \mathrm{~EB}+\text { rabbit }^{*} \mathrm{VH}+ \\
\text { rabbit }^{*} \mathrm{QL} 1\end{array}$ & 16 & 36787.2 & 15.4 & \\
\hline 9 & fox & 10 & 36787.6 & 15.8 & $<0.01$ \\
\hline \multirow[t]{4}{*}{10} & $\mathrm{VH}$ & 10 & 36787.7 & 15.9 & $<0.01$ \\
\hline & $\mathrm{VH}+\mathrm{QL} 1+\mathrm{QL} 1 * \mathrm{VH}$ & 12 & 36790.4 & 18.6 & \\
\hline & fox + rabbit + fox* rabbit & 12 & 36790.4 & 18.6 & \\
\hline & $E B+$ fox + fox*EB & 12 & 36791.3 & 19.5 & \\
\hline 11 & QL1 & 10 & 36793.9 & 22.1 & $<0.01$ \\
\hline \multirow[t]{6}{*}{12} & intercept & 9 & 36794.0 & 22.2 & $<0.01$ \\
\hline & rabbit & 10 & 36794.7 & 22.8 & \\
\hline & EB & 10 & 36795.8 & 24.0 & \\
\hline & $\mathrm{QL} 1$ + rabbit + rabbit*QL1 & 12 & 36796.6 & 24.8 & \\
\hline & $\mathrm{EB}+\mathrm{QL} 1+\mathrm{QL} 1 * \mathrm{~EB}$ & 12 & 36797.9 & 26.1 & \\
\hline & $\mathrm{EB}+$ rabbit + rabbit ${ }^{*} \mathrm{~EB}$ & 12 & 36798.1 & 26.3 & \\
\hline
\end{tabular}

AICc $=$ Aikaike information criterion corrected for small sample size; $\triangle \mathrm{AICc}=$ delta AICc with regard to best fitting model; $\mathrm{w}_{\mathrm{i}}=$ Akaike weight or relative weight of each model. $\dagger$ Only parsimonious models were weighted, i.e., more complex models with lower AICc (shaded) were left out. Model parameters: QL1 $=1^{\text {st }}$ PCA component of forage quality: $\mathrm{N}$ and P; QL2 $=2^{\text {nd }}$ PCA component of forage quality: $\mathrm{NDF}(+)$ and $\mathrm{Ca}(-) ; \mathrm{EB}=$ edible biomass $\left(\mathrm{g} \cdot \mathrm{m}^{-2}\right)$; fox $=$ red fox activity; rabbit = rabbit activity. All models contained the control variables area type and Sex. Models are based on: 2843 observations of 11 hare in 19 vegetation types in 2 areas over 79 days. 



\section{CHAPTER 5}

Chronic exposure to high predation risk of multiple predators decreases body condition and reproductive output of European hare

Weterings, M.J.A., Losekoot, S., Kuipers, H.J., Prins, H.H.T., Van Langevelde, F., Van Wieren, S.E.

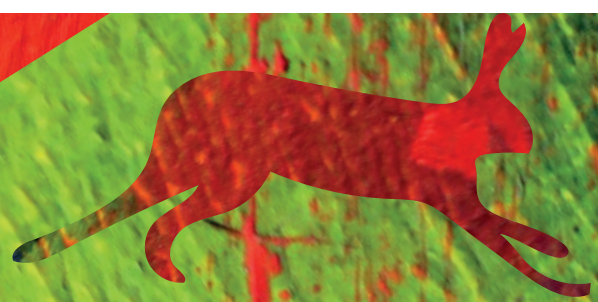




\section{ABSTRACT}

Chronic exposure to high predation risk is thought to directly suppress body condition and reproductive output of prey. We tested the expected negative correlation between chronic exposure to predation risk of multiple predators and body condition and reproductive output of a medium-sized prey species, the European hare, from 13 areas in The Netherlands. We estimated the density of all predators year-round present in the areas and expressed chronic exposure to predation risk as the sum of their field metabolic rates (FMR). The field metabolic rate reflects the daily energy requirements of an individual. To describe body condition, we extracted four components of the measurements of shot hares by a principal component analysis that resulted in proxies for 'health', 'age', 'size', and 'chronic stress'. We counted the number of placental scars to estimate reproductive output. As expected, we found that FMR of predators was negatively correlated with the number of placental scars and the 'health' component of body condition. Our paper reports for the first time a negative correlation between chronic exposure to predation risk of multiple predators and the reproductive output of a mediumsized mammal prey species under field conditions. With our findings, our paper contributes to a better understanding of the risk effects of predation on prey fitness that are often suggested but hardly tested in field studies. 


\section{INTRODUCTION}

Chronic exposure to predation risk has important effects on body condition and reproductive output of prey species (Hawlena \& Schmitz, 2010; Zanette et al., 2014). Prey encounters with a predator trigger anti-predator behaviour and increase energetic or physiological costs to prey, which may negatively affect prey fitness (Preisser et al., 2005; Creel \& Christianson, 2008; Sheriff et al., 2009). These stress responses help prey to cope with risky situations by 'minimizing or shutting down non-essential functions, mobilizing energy, and facilitate escape from life-threatening situations' (Sheriff et al., 2011, p.594). Chronic exposure to stress (e.g., caused by continuous predation risk) is thought to directly suppress the reproductive output of prey (Krebs et al., 2001) to benefit survival (Bian et al., 2015). Additionally, chronic exposure to stress can be passed on to the next generation through a maternal effect (Boonstra et al., 1998), having prolonged demographic consequences. Besides loss in reproductive output, chronic exposure to stress can negatively affect body condition (Boonstra et al., 1998; Van Dievel et al., 2015).

Few prey species, however, are affected by single predator only (Sih et al., 1998). In a general food web, each prey species has about two to three predators preying on them (Schoener, 1989), whereas predator density is constrained by metabolic rates and prey biomass (Carbone \& Gittleman, 2002). Besides, complex trophic webs (e.g., the terrestrial ecosystem) contain a high fraction of omnivores and generalist predators (Strong, 1992) that together with specialist predators can enhance the stress response of prey (Frid \& Dill, 2002; Koops, 2004). In addition, human hunting can elicit prey behavioural responses similar to risk associated with predators (Proffitt et al., 2009), probably with similar stress responses (Ciuti et al., 2012).

Multiple-predator effects on prey species depend on the functional characteristics of the predators, and can be difficult to investigate in field situations (Schmitz, 2007). However, behavioural decisions and behavioural syndromes (sensu Sih et al., 2004) of predators, food requirement, food intake and predation attack rates are related to the metabolic rate of predators (Nagy et al., 1999; Brown et al., 2004; Carbone \& Gittleman, 2002; Careau et al., 2008). We will therefore use the sum of the field-metabolic rate (FMR) of all predators in an area to represent predation risk for prey, which integrates the variation in predator body mass, predator type and the density of multiple predators to reflect the daily energy requirements of all predators (Carbone \& Gittleman, 2002). Sinclair et al. (2003) and Hopcraft et al. (2010) propose that medium-sized herbivores are thought to be especially vulnerable to predation. Because medium-sized herbivores are strongly regulated by predation risk, we expect that this type of herbivore should show strong negative effects on body condition and reproductive output with 
increasing risk from multiple predators. Our objective was thus to test the negative correlation between chronic exposure to predation risk of multiple predators and body condition and reproductive output of a medium-sized prey species, the European hare (Lepus europaeus).

\section{METHODS}

\section{Study area}

We conducted the study in 13 hunting leases $(\bar{X} \pm S D=663 \pm 551$ ha; Appendix 5, table A5.1) distributed over the Netherlands (figure 5.1) in three types of areas: sandy, sea clay and riverine clay areas. Compared to clay areas, sandy areas in general are drier and contain a lower concentration of soil nutrients (Bal et al., 2001). Selection of hunting leases was based on the voluntary participation of hunters in response to an invitation in the national club magazine of The Dutch Hunters Association (NJV). Hunting leases are comprised of a set of subareas on which hunting of local wildlife is managed and coordinated by a local group of hunters who together lease the local right to hunt from landowners. Subareas are homogeneous patches of vegetation types (mainly crops and pasture) or ploughed areas.

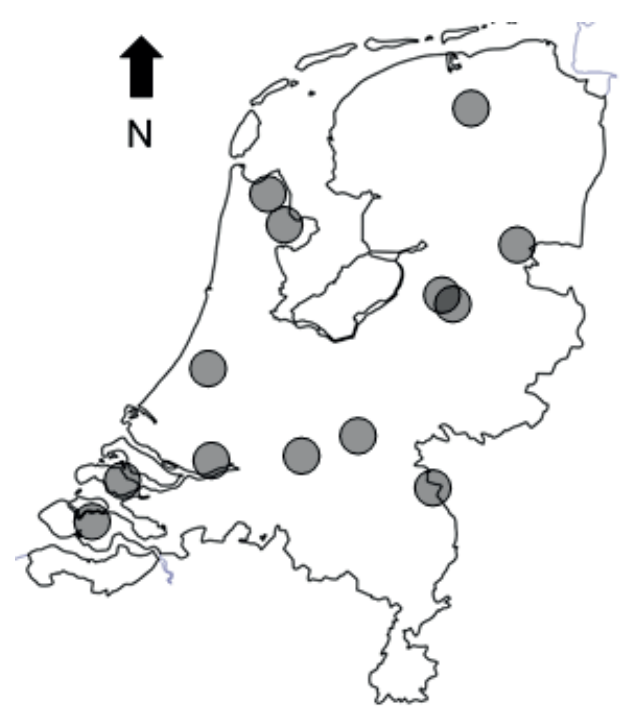

Figure 5.1: Distribution of the investigated hunting leases in the Netherlands with European hare (Lepus europaeus).

The characteristics of hunting leases can be found in Appendix 5. 


\section{Data collection}

Between 14 November and 17 December 2013 we collected 73 hares that were shot at 14 hunts $(\bar{X} \pm \mathrm{SD}=5.6 \pm 2.8$ hares/hunt $)$ in the hunting leases. Hares were hunted by foot or driven towards hunters at fixed positions. Drives consisted of hunters and beaters with or without dogs. We accompanied the hunters in the field and counted the number of hares flushed and shot, to calculate hare density and the percentage of hares shot. Experienced hunters $(\bar{X} \pm \mathrm{SD}=31 \pm 14$ years of hunting experience) that surveyed their hunting leases weekly $(\bar{X} \pm \mathrm{SD}=6 \pm$ $3 \mathrm{~h} /$ week) were interviewed to estimate the year-round abundance and presence of 25 potential predator species of hares active on their hunting lease during the last year (Appendix 5, table A5.2). Hares (especially when they are young) can be predated by multiple predators, such as foxes, birds of prey and members of the mustelid family (Huber, 2004; Tapper \& Yalden, 2010). After the hunt, we took a random subset $(\bar{X} \pm \mathrm{SD}=5.6 \pm 2.8$ hares $/$ hunt $)$ of shot hares that were offered by the hunters. Hares were stored at low temperatures $\left(<7^{\circ} \mathrm{C}\right)$ and dissected within 1 to 4 days ( $\bar{X} \pm \mathrm{SD}=1.8 \pm 0.8$ days) after the hunt.

We conducted a general health assessment of sampled hares before and during dissection, by assessing the presence of parasites, as well as lesions and other abnormalities. Tissue samples of liver, lung, spleen, brain, heart and kidneys were examined by the Dutch Wildlife Health Centre (DWHC) for general pathology. Our general health assessment indicated that in $7.5 \%$ of the carcasses investigated no abnormalities were found. $83 \%$ of the hares had medical abnormalities of minor importance, of which nephritis in kidneys $(n=17)$, inflammatory infiltrates $(\mathrm{n}=15)$, hepatitis $(\mathrm{n}=8)$ and pneumonia $(\mathrm{n}=7)$ were most often found. We found that $9.5 \%$ of the hares had medical abnormalities greater than minor importance, such as liver flukes $(\mathrm{n}=3)$, pseudotuberculosis $(\mathrm{n}=2)$ and encephalitis $(\mathrm{n}=2)$. Only two ecto-parasites (i.e., ticks only) were encountered on two different individuals.

To assess body condition, we measured hare body mass (dry fur weight), zygomatic skull width (maximum width at orbital cavity) following Bray et al. (2002), ear length, hind-foot length and weight of the heart, liver and kidneys (Appendix 5, table A5.3). Eye lenses were removed to determine age, and stored in $10 \%$ formalin solution. After 29.6 days \pm 9.1 (SD) since first storage, we air-dried the eye-lenses at $80^{\circ} \mathrm{C}$ for 6 days. Eye-lenses were weighed to the nearest 0.1 gram. Age in months was calculated using the equation reported in Broekhuizen and Maaskamp (1979) (equation 1). 
[Equation 1] Age (months):

$A G E=\frac{e^{\left(\frac{W+222.2}{83.3}\right)}}{30}$

$W \quad=$ eye-lens weight (in $\mathrm{mg})$

Adrenal glands were carefully removed and weighed without adhering tissue as an estimator of chronic stress (Harder \& Kirkpatrick, 1994). Uteri were removed to count the number of placental scars as an estimator of reproductive output. As uterine walls of European hare regenerate during anoestrus, placental scar counts represent the number of embryos that had developed during the last year since the start of the breeding season halfway February (Bray et al., 2003). European hares have up to 5 litters each year, with a mean litter size between 2 and 3 leverets (Marboutin et al., 2003), whereas annual reproductive output is quite constant ( $\pm 10-11$ placental scars) across regions (Hackländer et al., 2011). Placental scars were counted at the University of Natural Resources and Life Sciences, Vienna (BOKU) by Martijn Weterings and Klaus Hackländer following the protocol by Hackländer et al. (2001). Weterings and Hackländer independently assessed, discussed and verified the number of scars, using a 7-30x magnification zoom stereoscopic binocular.

To assess validity of hunter estimates of predator numbers, we used independently collected data from Dutch mammal (NDFF, 2015) and bird species distribution maps (SOVON, 2017). For each predator species we summed the number of 'Atlas blocks' (i.e., $5 \times 5 \mathrm{~km}$ blocks) that were occupied by the species in a $3 \times 3$ matrix (range: 0-9) around the hunting leases.

\section{Data preparation}

Chronic exposure to predation risk was expressed as the total field metabolic rate (FMR) of all potential avian and mammalian predators of hares present in a hunting lease during the last year. We used FMR as a 'fundamental biological rate' (Brown et al., 2004, p.1771) to represent predation risk. The total FMR in a hunting lease reflects the daily energy requirements of these predators taking into account predator body mass, predator type and the density of multiple predators. Hunter reports of predator numbers are often overestimated (Filion, 1980; Padding \& Royle, 2012), especially when estimating a large number of individuals (Wright, 1978). To correct for this possible bias, we assigned the abundance of each predator species to the lower limit of one of 11 abundance categories doubling in number $(1,2,3,4-8,8-16,16-32,32-64,64-128,128-256,256-512$, 512-1024). Predator type specific allometric relationships by Nagy et al. (1999) were then used to calculate the FMR per predator species in each hunting lease 
(equation 2). First we assigned each predator to a specific predator type (Nagy et al., 1999, see also Appendix 5, table A5.2), and substituted the average of the lower and higher limit of their body mass (birds: Del Hoyo et al., 1992; 1994; 1996; 1999; 2009; mammals: Lange et al., 2003) to calculate $F_{M R}$ BMavg. $_{\text {. }}$ Second, we assigned the hunter estimates to the lower limit of their abundance category, and calculated the proportion of the year each species (i.e., birds only) was resident in the Netherlands (Vogelbescherming, 2017).

Field metabolic rate (FMR) per predator species for each hunting lease (KJ day ${ }^{-1}$ $\left.h a^{-1}\right)$ :

[Equation 2]

$$
F M R=F M R_{B M_{\text {avg }}} *\left(\frac{A b_{l o w^{* P}}}{A}\right)
$$

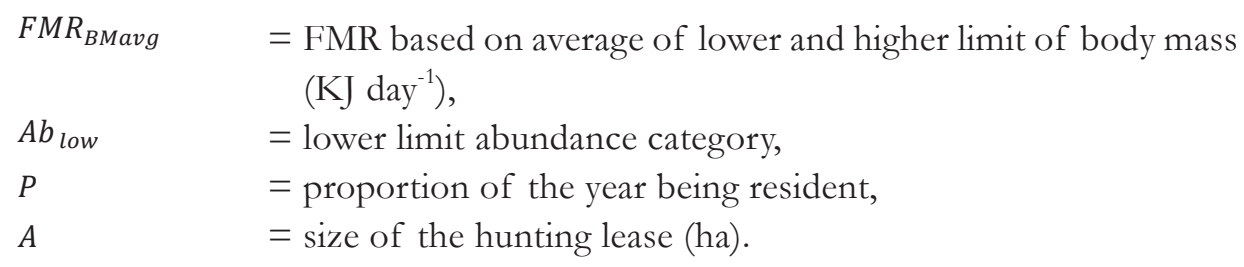

We also investigated the effect of hunting risk, to be able to assess its relative effect compared to predation risk, as prey responses to hunting can be stronger than responses to predators (Proffitt et al., 2009). Hunting risk was expressed as the percentage of hares shot in a hunting lease. Hunts were restricted to shooting in the period between 15 October and 31 December, with a frequency between 1 and 5 hunts $(\mathrm{n}=8)$. We assumed that hunting risk did not change between years, based on our communications with the local hunting groups.

To describe body condition, we performed a principal component analysis (SPSS version 23.0) upon ear length, hind-foot length, body weight, zygomatic width, eye-lens weight, kidney weight, heart weight, liver weight and weight of adrenalgland pair, and extracted four principal components. Before factor analysis all body measurements were $\log _{10}$ transformed, because of the power functions expected for allometric relationships (Peters, 1983), and standardized, as factor analysis is not scale invariant. Components were rotated by a Varimax with Kaiser Normalization. Components with an eigenvalue above 1 (Kaiser, 1960) were calculated and standardized by the Anderson-Rubin method, which ensures orthogonality of the estimated factors (DiStefano et al., 2009). 


\section{Data analysis}

Although multiple-predator effects may be non-linearly related to prey response (Griffin et al., 2015), we only focussed on linear effects on body condition and reproductive output. Linear effects were expected, because hare has a broad habitat domain (i.e., is widely distributed), whereas its predators, which differ in hunting mode, have a broad $(48 \%, \mathrm{n}=25$, distributed over more than $50 \%$ of the Dutch area) or a narrow (52\%) habitat domain (Schmitz, 2007).

We investigated the effect of predation and hunting risk on three components of body condition (i.e., all except the second component 'age'; see results), by running linear mixed models in $\mathrm{R}$ (package lme4 version 1.1-12). We investigated the effect of predation and hunting risk on the number of placental scars by running generalized linear mixed models in $\mathrm{R}$ (package lme4 version 1.1-12), with a binomial error structure and logit link. A maximum of 19 placental scars was used as the upper limit of the binomial distribution based on literature (Hackländer, 2001; Smith et al., 2010) and best fit. The following females were excluded from placental scar analysis: females with inactive uteri (i.e., too small for reproduction) ( $\mathrm{n}=13 ; 1$ adult, 12 sub adults), females with active uteri that did not reproduce ( $\mathrm{n}=3 ; 1$ adult, 2 sub adults), as well as females of which the uterus contained tumours or other abnormalities ( $\mathrm{n}=3 ; 2$ adults, 1 sub adult). Additionally we assessed the effect of the four PCA components of hare body measurements on the number of placental scars, as we expected body condition (Travers et al., 2010) including age (Hackländer et al., 2001; Stott \& Harris, 2006; Smith et al., 2010) to affect reproductive output.

Hunter effort (i.e., hours spent in hunting lease per week) and the type of area (i.e., physical geographical region) were used as control variables for all candidate models. We included the type of area, because stress responses can be the result of chronic exposure to abiotic or biotic stressors (Sheriff \& Thaler, 2014) related to the type of area such as soil type and food, which also can affect body condition (Krebs et al., 2001). Sex and days since the start of the field data collection were used as control variables for the models with body condition only. The latter was included because hares fatten up within several weeks at the end of the year to prepare for the next breeding season (Valencak et al., 2009; see also seasonal variation in kidney weight: Van Vuuren \& Coblentz, 1985).

We used standardized regression coefficients to assess the effect of the predictor variables on the response variables. Continuous predictor variables were standardized and scaled by dividing their mean by two standard deviations (Gelman, 2008). FMR and hunter effort was $\log _{10}$ transformed to normalize a right skewed distribu- 
tion. Multicollinearity of continuous predictor variables was assessed by a script from Zuur et al. (2010). The Variance Inflation Factor (VIF) of all continuous predictor variables remained below 1.3 for both datasets. All candidate models included hunting lease as a random factor, with subareas nested within hunting lease.

To assess the effects of control variables, we started with two global models that included all control variables (i.e., model 1: predation risk + all control variables, and model 2: hunting risk + all control variables). We then dredged both models keeping the predictor variables predation and hunting risk in each model. Parsimonious models only were selected for two model sets. Betas of predictors were estimated using conditional model averaging of both model sets combined. Over and under dispersion of models with count data was assessed by the Pearson Chi-square over the residual degrees of freedom of the model, whereas the fit of these models was assessed by their relative deviance (Crawley, 2007).

To validate hunter estimates, we correlated the species distribution from atlases, hare density, hunter effort, predator species body mass, the proportion of the year predators that are resident, and the size of the hunting lease with the density of predators estimated by the hunters. Predator density was fourth-root transformed to correct for skewed data. We ran linear mixed models in $\mathrm{R}$ (package lme4 version 1.1-12), with predator species as a random factor. Hare density was used to explain hunter estimates, because prey density is related to the maximum number of predators (Carbone \& Gittleman, 2002). Additionally, the body mass of predator species was taken into account, because detection of species is positively related to body mass (Rowcliffe et al., 2011). The global model was dredged and ranked by AICc. Parsimonious models only were selected for full-model conditional averaging.

\section{RESULTS}

Four components of the PCA explained $77.6 \%$ of the total variation in all body measurements (table 5.1). Kidney weight, heart weight and liver weight were strongly correlated (coefficient $>0.7$ ) with the first component, whereas zygomatic width and eye-lens weight were strongly correlated with the second component. Average ear length and average hind-foot length were strongly correlated with the third component, whereas the weight of the adrenal-gland pair was the only body measure that strongly correlated with the fourth component. Body weight was correlated with three components. We characterized the first component as a 'health' axis, based on its strong correlation with organ weights, the second as an 'age' axis, the third as a 'size' axis and the fourth as an axis of 'chronic stress'. 
Table 5.1: Rotated component coefficient values of hare body measurements $(n=57)$.

\begin{tabular}{lcccc}
\hline & \multicolumn{4}{c}{ Component $^{1}$} \\
\cline { 2 - 5 } Body measurements $^{2}$ & 1 & 2 & 3 & 4 \\
\hline Ear length (avg.)(cm) & $38.2 \%(3.4)^{3}$ & $15.4 \%(1.4)$ & $13.0 \%(1.2)$ & $10.9 \%(1.0)$ \\
Hind foot length (avg.)(cm) & -0.16 & 0.05 & $\mathbf{0 . 8 1}$ & 0.15 \\
Body weight (g) & 0.26 & 0.09 & $\mathbf{0 . 7 4}$ & -0.11 \\
Zygomatic width (mm) & 0.50 & 0.61 & 0.46 & -0.13 \\
Eye-lens weight (avg.)(mg) & -0.08 & $\mathbf{0 . 8 5}$ & 0.10 & -0.12 \\
Kidney weight (avg.)(g) & 0.31 & $\mathbf{0 . 8 2}$ & 0.03 & 0.16 \\
Heart weight (g) & $\mathbf{0 . 7 1}$ & 0.44 & -0.05 & 0.12 \\
Liver weight (g) & $\mathbf{0 . 7 9}$ & 0.43 & 0.12 & 0.06 \\
Adrenal weight (pair)(mg) & $\mathbf{0 . 8 4}$ & -0.20 & 0.05 & 0.03 \\
\hline & 0.09 & -0.01 & 0.03 & $\mathbf{0 . 9 7}$ \\
\hline
\end{tabular}

\footnotetext{
${ }^{1}$ PCA coefficient values $>0.7$ are bold; Varimax with Kaiser Normalization; list-wise deletion; ${ }^{2} \log _{10}$ transformed and standardized, pairs of measurements are averaged; ${ }^{3}$ Percentage of variance explained by component (eigenvalue of component); ${ }^{4}$ Protocol by Broekhuizen and Maaskamp (1979), eye-lens weight air dried at $80^{\circ} \mathrm{C}$ for 6 days.
}

Four species had an above average FMR density $\left(>44.4 \mathrm{KJ} \mathrm{day}^{-1} \mathrm{ha}^{-1}\right)$ in the hunting leases investigated, namely grey heron (Ardea cinerea), domestic or feral cat (Felis catus), red fox (Vulpes vulpes) and Eurasian buzzard (Buteo buteo) (figure 5.2, Appendix 5, table A5.2). Especially the presence of these species, with a large average body mass and a large estimated density, may result in a high year-round predation risk for the hares. The FMR of predators and the percentage of hares shot were negatively correlated with the first component of body condition (table 5.2). A high FMR of predators, or a high percentage of hares shot, correlated with a lower liver weight, heart weight, kidney weight, and body weight. Area type was correlated with the first and fourth component of body condition. Organ weight, body weight and adrenal weight of hares in sandy areas was greater than in clayey areas. Days since the start of the data collection was positively correlated with the first component of body condition, whereas hunter effort was negatively correlated with the third component of body condition. The percentage hares shot had no effect on the third or fourth component of body condition or the number of placental scars. The number of placental scars was negatively correlated with the FMR of predators (table 5.3) (figure 5.3). The PCA components of hare body measurements had no correlation with the number of placental scars. 


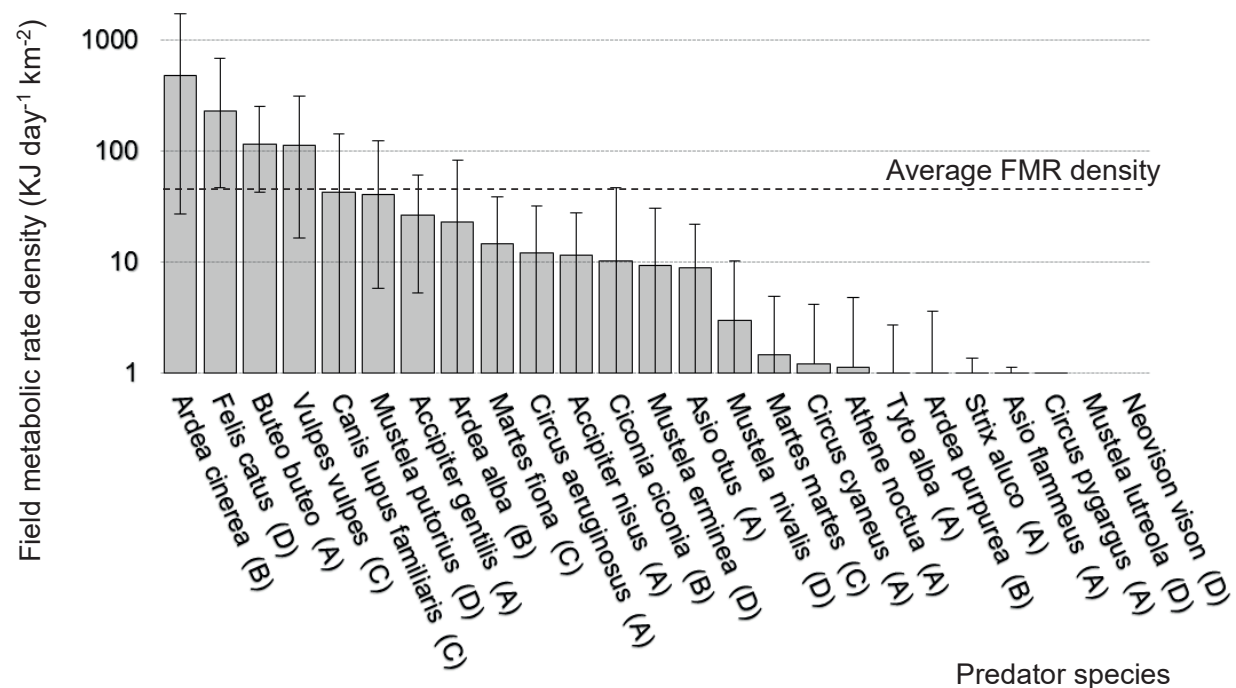

Figure 5.2: Field metabolic rate density $(\bar{X} \pm 95 \% C I)$ for predators of European hare (Lepus europaeus) in Dutch hunting leases $(n=13)$.

Predator types based on Nagy et al. (1999): A = all birds, B = Pelecaniformes, C = mammal carnivores, $\mathrm{D}=$ mammal omnivores. Field metabolic rate density $=$ weighted species density $*$ average species field metabolic rate (i.e., a measure of chronic exposure to predation risk). The weighted species density $=$ species numbers estimated by hunters weighted by the size of the hunting lease and multiplied by the proportion of the year that the species is present (see text for further explanation). Note the logarithmic scale of the y-axis.

Hunter estimates of predator density were positively correlated with predator species distribution and proportion of the year the predator was resident, whereas the size of the hunting lease was negatively correlated with predator density (Appendix 5, table A5.4). Hunter estimates were not correlated with hunter effort and hare density. 
Table 5.2: Results of the linear mixed regression of the effect of predation risk (FMR, field metabolic rate) and hunting risk (PER, percentage shot) on three PCA components of body measurements of the European hare $(n=54)$.

\begin{tabular}{|c|c|c|c|c|c|c|}
\hline & Component 1 'Health' & & & & & \\
\hline No. & Model type $^{1}$ & Control variables $^{2}$ & $d f$ & $\mathrm{AICc}^{3}$ & $\triangle \mathrm{AICc}$ & $\mathrm{w}_{\mathrm{i}}^{4}$ \\
\hline 1 & $\log _{10}$ FMR & DAY + AREA type & 8 & 144.9 & 0.0 & 0.65 \\
\hline 2 & $\log _{10}$ FMR & DAY & 6 & 147.4 & 2.4 & 0.19 \\
\hline 3 & PER & DAY & 6 & 148.8 & 3.9 & 0.09 \\
\hline 4 & $\log _{10}$ FMR & & 5 & 151.1 & 6.1 & 0.03 \\
\hline 5 & PER & & 5 & 151.6 & 6.7 & 0.02 \\
\hline \multirow[t]{10}{*}{6} & Intercept & & 4 & 152.2 & 7.3 & 0.02 \\
\hline & Variables & Estimate $\left(\beta \pm \widehat{\mathrm{SE}}_{\beta}\right)^{5}$ & $Z_{\text {value }}{ }^{6}$ & \multicolumn{2}{|c|}{$2.5 \%-97.5 \%$ C.I. } & $\mathrm{W}_{\mathrm{p}}{ }^{7}$ \\
\hline & $\log _{10}$ FMR & $-0.8 \pm 0.2$ & $3.3^{*}$ & \multicolumn{2}{|c|}{$-1.33--0.35$} & 0.87 \\
\hline & PER & $-0.6 \pm 0.3$ & $2.3^{*}$ & \multicolumn{2}{|c|}{$-1.14--0.09$} & 0.12 \\
\hline & \multicolumn{6}{|l|}{ AREA type ${ }^{8}$} \\
\hline & Sea clay areas & $-0.8 \pm 0.3$ & $2.7^{*}$ & \multicolumn{2}{|c|}{$-1.38--0.21$} & 0.65 \\
\hline & Riverine clay areas & $-1.0 \pm 0.4$ & $2.4^{*}$ & \multicolumn{2}{|c|}{$-1.73--0.17$} & 0.65 \\
\hline & DAY & $0.9 \pm 0.3$ & $2.8^{*}$ & \multicolumn{2}{|c|}{$0.26-1.47$} & 0.93 \\
\hline & Intercept & $0.5 \pm 0.4$ & 1.2 & \multicolumn{2}{|c|}{$-0.29-1.28$} & 1.00 \\
\hline & \multicolumn{6}{|l|}{ Component 3 'Size' } \\
\hline No. & Model type & Control variables & $d f$ & $\mathrm{AICc}$ & $\triangle \mathrm{AICc}$ & $\mathrm{w}_{\mathrm{i}}$ \\
\hline 1 & PER & EFFORT & 6 & 158.9 & 0.0 & 0.45 \\
\hline 2 & Intercept & & 4 & 160.1 & 1.2 & 0.25 \\
\hline 3 & PER & & 5 & 160.3 & 1.3 & 0.23 \\
\hline \multirow[t]{6}{*}{4} & $\log _{10}$ FMR & & 5 & 162.4 & 3.4 & 0.08 \\
\hline & Variables & Estimate $\left(\beta \pm \widehat{\mathrm{SE}}_{\beta}\right)$ & $\mathrm{Z}$ value & \multicolumn{2}{|c|}{$2.5 \%-97.5 \%$ C.I. } & $\mathrm{W}_{\mathrm{p}}$ \\
\hline & $\log _{10}$ FMR & $0.1 \pm 0.3$ & 0.7 & \multicolumn{2}{|c|}{$-0.45-0.73$} & 0.08 \\
\hline & PER & $0.5 \pm 0.3$ & 1.8 & \multicolumn{2}{|c|}{$-0.06-1.02$} & 0.67 \\
\hline & EFFORT & $-0.5 \pm 0.3$ & $2.0^{*}$ & \multicolumn{2}{|c|}{$-1.06--0.02$} & 0.45 \\
\hline & Intercept & $0.1 \pm 0.1$ & 0.4 & \multicolumn{2}{|c|}{$-0.22-0.33$} & 1.00 \\
\hline
\end{tabular}


Table 5.2 continued

Component 4

'Chronic stress'

\begin{tabular}{|c|c|c|c|c|c|c|}
\hline No. & Model type & Control variables & $d f$ & $\mathrm{AICc}$ & $\triangle \mathrm{AICc}$ & $\mathrm{w}_{\mathrm{i}}$ \\
\hline 1 & $\log _{10}$ FMR & SEX & 6 & 157.1 & 0.0 & 0.31 \\
\hline 2 & $\log _{10} \mathrm{FMR}$ & AREA type & 7 & 158.6 & 1.5 & 0.15 \\
\hline 3 & Log10 FMR & & 5 & 158.6 & 1.5 & 0.15 \\
\hline 4 & Intercept & & 4 & 159.4 & 2.3 & 0.10 \\
\hline 5 & PER & AREA type + DAY & 8 & 159.8 & 2.8 & 0.08 \\
\hline 6 & PER & AREA type + EFFORT & 8 & 160.0 & 3.0 & 0.07 \\
\hline 7 & PER & AREA type & 7 & 160.5 & 3.5 & 0.06 \\
\hline 8 & PER & SEX & 6 & 161.2 & 4.6 & 0.03 \\
\hline 9 & PER & EFFORT & 6 & 161.8 & 4.7 & 0.03 \\
\hline 10 & PER & & 5 & 161.8 & 4.8 & 0.03 \\
\hline & Variables & Estimate $\left(\beta \pm \widehat{S E}_{\beta}\right)$ & $Z$ value & \multicolumn{2}{|c|}{$2.5 \%-97.5 \%$ C.I. } & $\mathrm{W}_{\mathrm{p}}$ \\
\hline & $\log _{10}$ FMR & $0.5 \pm 0.3$ & 1.9 & \multicolumn{2}{|c|}{$-0.01-1.06$} & 0.61 \\
\hline & PER & $0.2 \pm 0.4$ & 0.7 & \multicolumn{2}{|c|}{$-0.46-0.96$} & 0.29 \\
\hline & DAY & $0.6 \pm 0.3$ & 1.8 & \multicolumn{2}{|c|}{$-0.04-1.22$} & 0.08 \\
\hline & $\operatorname{SEX}^{9}$ & $0.5 \pm 0.3$ & 2.0 & \multicolumn{2}{|c|}{$-0.001-1.02$} & 0.34 \\
\hline & \multicolumn{6}{|l|}{ AREA type ${ }^{8}$} \\
\hline & Sea clay areas & $-0.9 \pm 0.4$ & $2.4^{*}$ & \multicolumn{2}{|c|}{$-1.66--0.18$} & 0.35 \\
\hline & Riverine clay areas & $-0.9 \pm 0.6$ & 1.5 & \multicolumn{2}{|c|}{$-2.01-0.26$} & 0.35 \\
\hline & EFFORT & $-0.5 \pm 0.3$ & 1.7 & \multicolumn{2}{|c|}{$-0.98-0.06$} & 0.10 \\
\hline & Intercept & $0.2 \pm 0.5$ & 0.4 & \multicolumn{2}{|c|}{$-0.78-1.13$} & 1.00 \\
\hline
\end{tabular}

${ }^{1}$ Models are based on measurements of 54 hares in 13 hunting leases collected over a period of 34 days; Model control variables (i.e., AREA type, DAY, EFFORT and SEX) are dredged, and ranking is based on parsimonious models only; Random factors: hunting lease/subarea; ${ }^{2}$ Control variables:, area type (AREA type), days since start of the data collection (DAY), hunter effort (EFFORT), and sex $(\mathrm{SEX}) ;{ }^{3} \mathrm{AICc}=$ Aikaike Information Criterion corrected for small sample size; ${ }^{4}$ wi $=$ Akaike weight or relative weight of each model; ${ }^{5}$ Estimates based on full-model conditional averaging of all parsimonious models; parameters are standardized by 2 SD (Gelman, 2008), ${ }^{6} *=95 \%$ confidence intervals do not include zero; ${ }^{7} \mathrm{Wp}$ : Akaike predictor weight; ${ }^{8}$ Sandy areas is reference category; ${ }^{9}$ Male is reference category. 
Table 5.3: Results of the generalized linear mixed models of the effect of predation risk (FMR, field metabolic rate), hunting risk (PER, percentage shot), and four PCA components of body measurements ('health', 'age', 'size' and 'chronic stress') on the number of placental scars of the European hare $(n=14)$.

\begin{tabular}{|c|c|c|c|c|c|c|}
\hline No. & Model type ${ }^{1}$ & $\frac{\mathrm{x}^{2}}{d f_{\text {res }}} 2$ & $d f$ & $\mathrm{AICc}^{3}$ & $\triangle \mathrm{AICc}$ & $\mathrm{w}_{\mathrm{i}}^{4}$ \\
\hline 1 & $\log _{10} \mathrm{FMR}$ & 1.4 & 4 & 72.6 & 0.0 & 0.93 \\
\hline 2 & Intercept & 0.7 & 3 & 79.4 & 6.8 & 0.03 \\
\hline 3 & Component 4 'chronic stress' & 0.7 & 4 & 80.2 & 7.6 & 0.02 \\
\hline 4 & Component 2 'age' & 0.5 & 4 & 82.3 & 9.7 & 0.01 \\
\hline 5 & PER & 0.8 & 4 & 82.7 & 10.1 & 0.01 \\
\hline 6 & Component 3 'size' & 0.8 & 4 & 83.2 & 10.7 & $<0.01$ \\
\hline \multirow[t]{9}{*}{7} & Component 1 'health' & 0.7 & 4 & 83.3 & 10.7 & $<0.01$ \\
\hline & Variables & Estimate $\left(\beta \pm \widehat{\mathrm{SE}}_{\beta}\right)^{5}$ & $Z_{\text {value }^{6}}$ & $2.5 \%-9$ & $7.5 \%$ C.I. & $\mathrm{W}_{\mathrm{p}}{ }^{7}$ \\
\hline & $\log _{10}$ FMR & $-1.3 \pm 0.3$ & $4.2^{*}$ & -1.95 & --0.70 & 0.93 \\
\hline & PER & $-0.5 \pm 0.6$ & 0.8 & -1.75 & -0.73 & 0.01 \\
\hline & Component 1 'health' & $-0.1 \pm 0.2$ & 0.3 & -0.50 & -0.35 & $<0.01$ \\
\hline & Component 2 'age' & $-0.2 \pm 0.2$ & 0.9 & -0.68 & -0.24 & 0.01 \\
\hline & Component 3 'size' & $0.1 \pm 0.1$ & 0.4 & -0.26 & -0.39 & $<0.01$ \\
\hline & Component 4 'chronic stress' & $0.3 \pm 0.1$ & 1.6 & -0.05 & -0.56 & 0.02 \\
\hline & Intercept & $0.3 \pm 0.1$ & 1.8 & -0.03 & -0.63 & 1.00 \\
\hline
\end{tabular}

${ }^{1}$ Models are based on measurements of 14 hares in 13 hunting leases collected over a period of 34 days; Random factors: hunting lease/subarea; ${ }^{2} \frac{x^{2}}{d f_{r e s}}=$ Pearson Chi-square over the residual degrees of freedom: close to 1 is no over- or under dispersion; ${ }^{3} \mathrm{AICc}=$ Aikaike Information Criterion corrected for small sample size; ${ }^{4}$ wi $=$ Akaike weight or relative weight of each model; ${ }^{5}$ Estimates based on full-model conditional averaging; parameters are standardized by $2 \mathrm{SD}$ (Gelman, 2008), ${ }^{6} *=95 \%$ confidence intervals do not include zero; ${ }^{7} \mathrm{Wp}$ : Akaike predictor weight. 


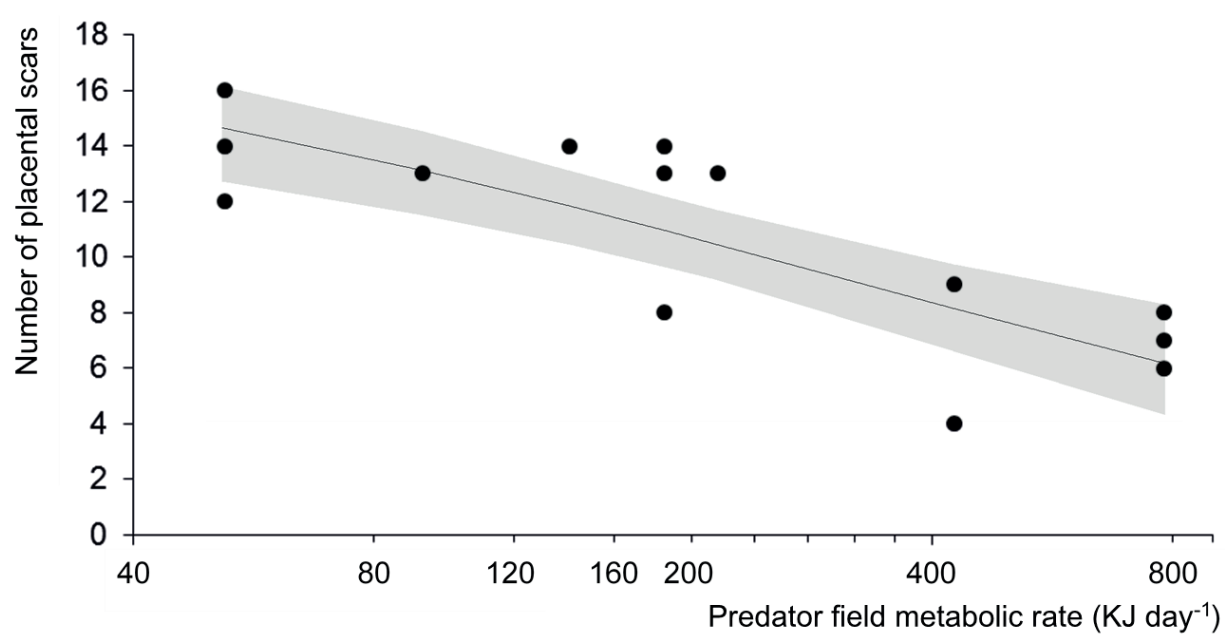

Figure 5.3: The relationship between the number of placental scars of the European hare (Lepus europaeus) and predator field metabolic rate as measure of chronic exposure to predation risk (points $=$ raw data, $\mathrm{n}=14$; line $=$ marginal effects of predicted probabilities of binomial model $\pm 95 \% \mathrm{CI}, \mathrm{Z}=4.7, \mathrm{df}=4, \mathrm{r}^{2}=0.83$ ).

\section{DISCUSSION}

We have tested the correlation between chronic exposure to predation risk and body condition and reproductive output of a medium-sized prey species in a field situation, by investigating the correlation between the field-metabolic rate (FMR) of predators and four components of body condition and the number of placental scars. Hawlena and Schmitz (2010) and Zanette et al. (2014) have reviewed 81 studies that investigated effects of predation risk on species behaviour, physiology or reproduction. The majority of these studies $(>86 \%)$ was experimental and involved the manipulation of (field) conditions or the capture of individuals to study effects on a single reproduction cycle (but see Monclús et al., 2011). Manipulation of field conditions is often necessary, because monitoring of reproductive success of crepuscular mammal species, especially non-central place foragers, is 'nearly impossible' (Sheriff et al., 2009, p1250). Our study is the first field study to correlate chronic exposure to risk of multiple predators to the reproductive output of a medium-sized mammal prey species. Our results are in line with studies that have demonstrated relationships between predation risk and reproductive output of animals (birds: Eggers et al., 2006, Zanette et al., 2011; mammals: Monclús et al., 2011; Sheriff et al., 2009; 2010; 2011), and can help to signify predation-risk effects (Zanette et al., 2014). As direct killing by predators 
is obvious, risk effects on prey fitness are much more difficult to measure in the field.

Any stressor that increases glucocorticoid concentrations can negatively affect reproductive output (Sheriff et al., 2009; Clinchy et al., 2011). Changes in physiology can explain the effect of chronic exposure to predation risk on reproductive output (Hawlena \& Schmitz, 2010; Travers et al., 2010; Zanette et al., 2013), whereas prey density, food availability, and disease and parasites have been investigated but were not found to affect reproductive output earlier (Krebs et al., 2001; but see Zanette et al., 2014). In our study, we did not find any indication that diseases and parasites were of such an importance that they would explain a reduction in reproductive output. A relationship between age and the reproductive output of hares has been reported earlier (Hackländer et al., 2001; Stott \& Harris, 2006; Smith et al., 2010). This is in contrast to our findings because we excluded non-reproducing females and included only one individual that was born in the year of investigation (Smith et al., 2010). Nevertheless, we could not find any relationship between the PCA components of body measurements and the number of placental scars, possibly because of the low sample size, because of annual variation in some of the body characteristics (e.g., body weight: Ebling \& Barrett, 2008, kidney weight: Van Vuuren \& Coblentz, 1985), or because we did not include energy reserves or other physiological stress measurements as indicators of body condition (Fernández et al., 2010; Travers et al., 2010; but see White et al., 2011). Although adrenal glands of mammals increase in size with chronic exposure to stress (e.g., Clark et al., 2006), we could not find any correlation between FMR and the 'chronic stress' component of body condition. Nevertheless, the 'chronic stress' component was larger in sandy areas, which could be related to a lower performance of herbivores in drier areas or areas with lower concentrations of soil nutrients (de Bruyn et al., 2002).

Behavioural responses of prey can be more strongly affected by hunting risk than risk induced by predators (Proffitt et al., 2009), but depend on the context (Ciuti et al., 2012). Consistent and strong harvest selection by hunters can even profoundly affect morphological and reproductive traits of species in a negative way (Darimont et al., 2009, but see Rivrud et al., 2013) or oppose natural selection (Carlson et al., 2007). We did not find any effect of hunting risk on reproductive output, possibly because of the restricted period in which hunting took place, or because of the effects of adverse weather (Van Wieren et al. 2006). However, body weight is known to be negatively affected by hunting pressure (Coltman et 
al., 2003; Tryjanowski et al., 2009; Szymanski et al., 2013), which corroborates with our results.

We estimated predation risk by the total FMR of predators reported by experienced hunters from hunting leases. FMR as an index of predation risk is generally preferred over predator density, abundance or related abundance indices (e.g., see Monclús et al., 2009, 2011; Sheriff et al., 2009), because FMR integrates the variation in predator body mass, predator type and the density of multiple predators to reflect the daily energy requirements of all predators (Carbone \& Gittleman, 2002). The use of FMR could be a promising methodology to investigate multi-predator effects on the body condition and reproductive output of prey. Although variation in $\log$ (body weight) within taxonomic classes accounts for $94 \%$ of the variation in $\log (\mathrm{FMR})$, residual variation in body weight adjusted FMR within taxonomic classes (e.g., related to life-stages of predators) can still be high (Nagy, 2005). Relative estimates of FMRs may not be affected by bias of hunter reporting rates, as the magnitude of the bias is expected to be constant (Padding \& Royle, 2012). Hunter estimates seemed valid as they were strongly positively correlated with independent data on predator species distribution, although hunters in smaller hunting leases reported higher predator densities. However, several aspects have not been investigated in this study. First, the presence of additional prey biomass will affect the diversity and density of predators (Carbone \& Gittleman, 2002). Changes in prey and predator community composition will alter various relationships between prey and its predators (Duffy et al., 2007), and thus also the risk perceived by prey. Second, effects of predators on prey species depend on hunting mode, habitat domain (Schmitz, 2007), interactions with other predators (Vance-Chalcraft \& Soluk, 2005; Lampropoulos et al., 2013), and resource specificity (Duffy et al., 2007) that can vary during the course of the season. We have not investigated how these factors affect chronic exposure to predation risk of hares during the course of the season. Predation risk of hares depends on their life stage. Young hares are affected by more different predators, and in different ways, than adult hares (Huber, 2004). Nevertheless, even species that disturb adult hares can trigger antipredator behaviour (Frid \& Dill, 2002), as a response to a potential predation risk (Koops, 2004).

Our paper reports a negative correlation between chronic exposure to predation risk of multiple predators and the reproductive output of a medium-sized mammal prey species in a field situation, which has not been done so far. Reduced body condition and hunting risk were not found to be negatively correlated with reproductive output. We suggest that field metabolic rate, which takes into ac- 
count density, predator type, body mass and energy requirements of multiple predators, can be a useful index of chronic exposure to predation risk. Future studies should incorporate the effect of alternate prey biomass on the effects of chronic exposure to predation risk by multiple predators on prey, as well as the variation in risk perceived by prey during different life-stages. With our findings, our paper contributes to a better understanding of the risk effects of predation on prey fitness that are often suggested but hardly tested in field studies. 


\section{APPENDIX 5}

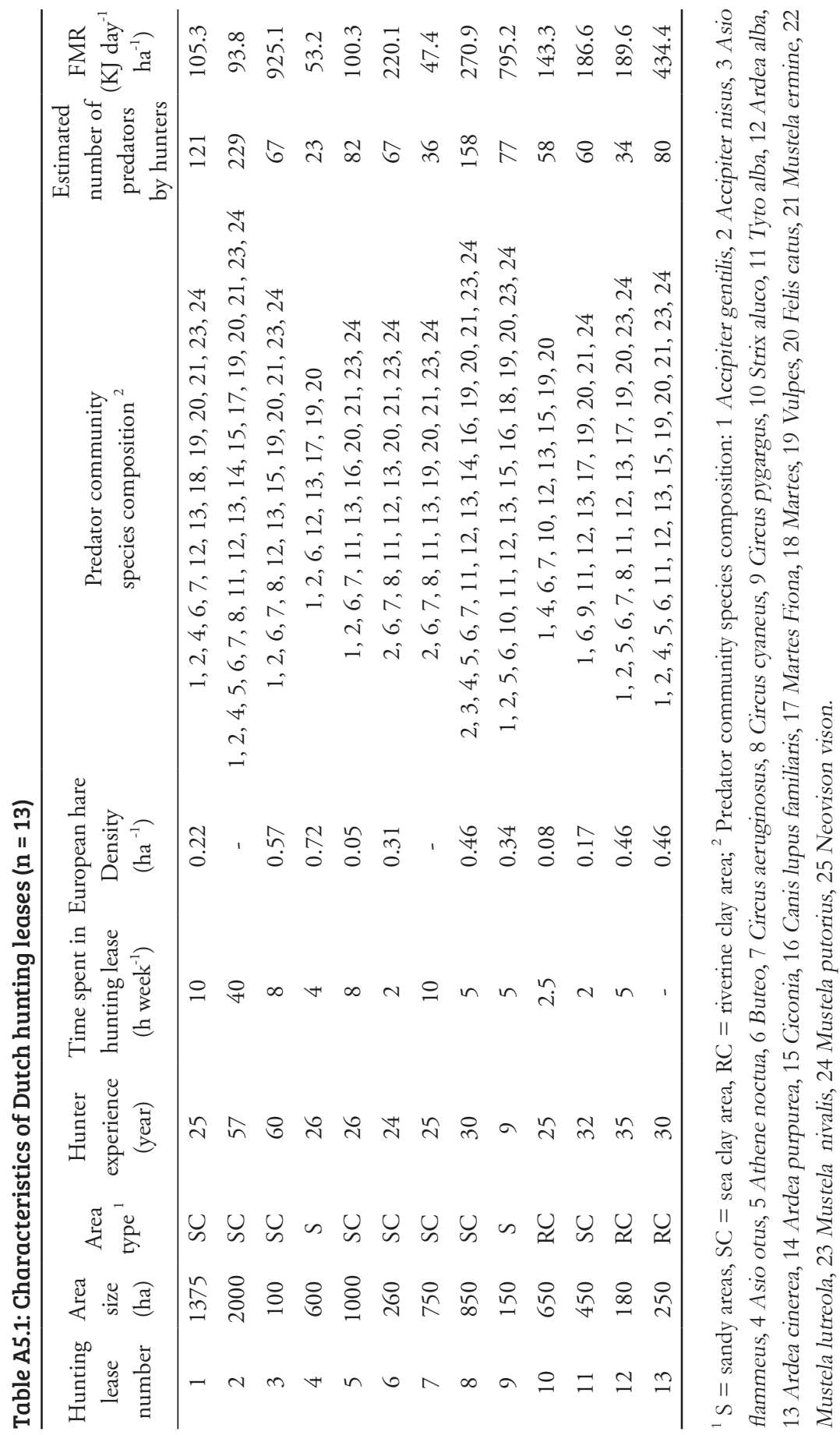




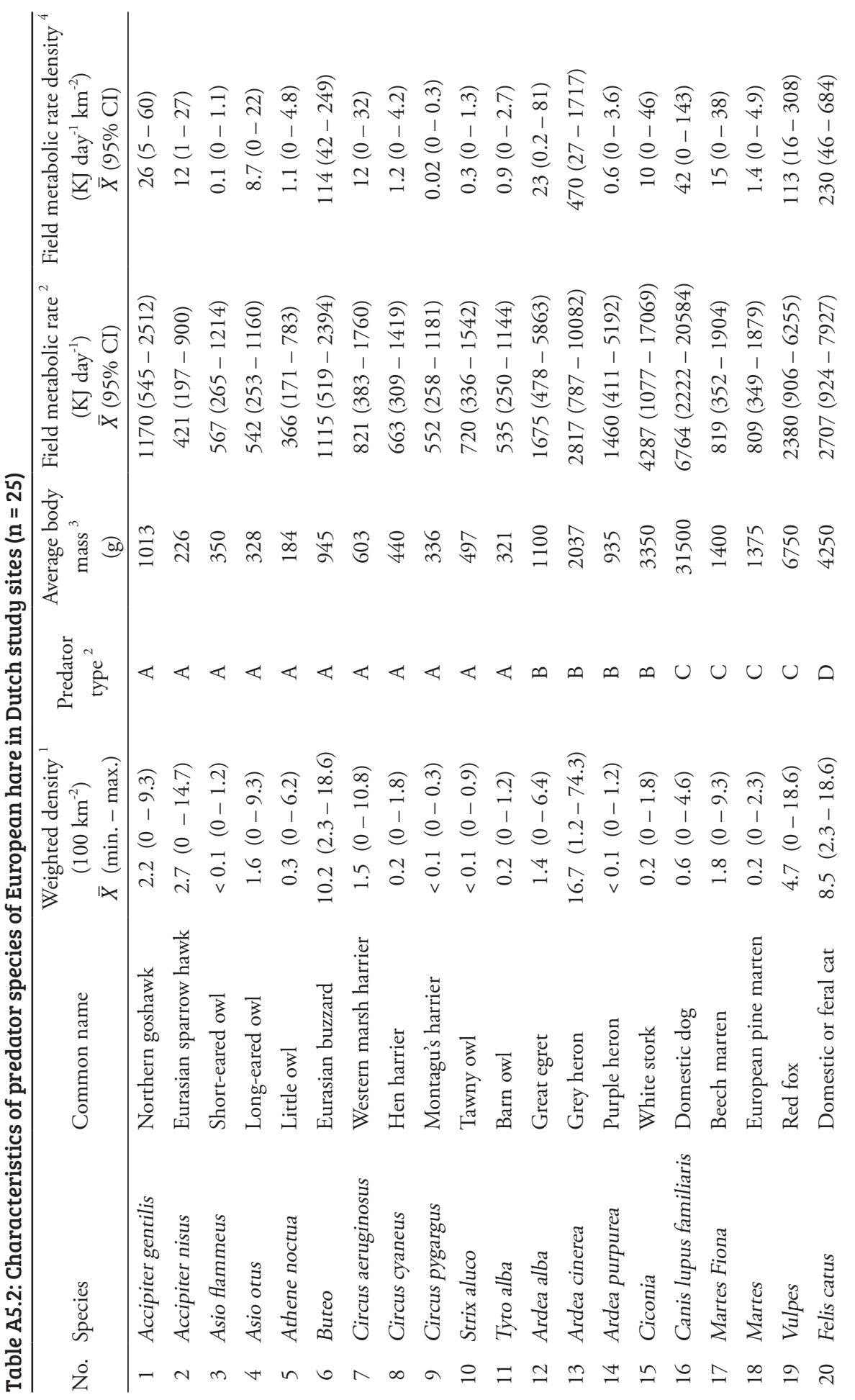




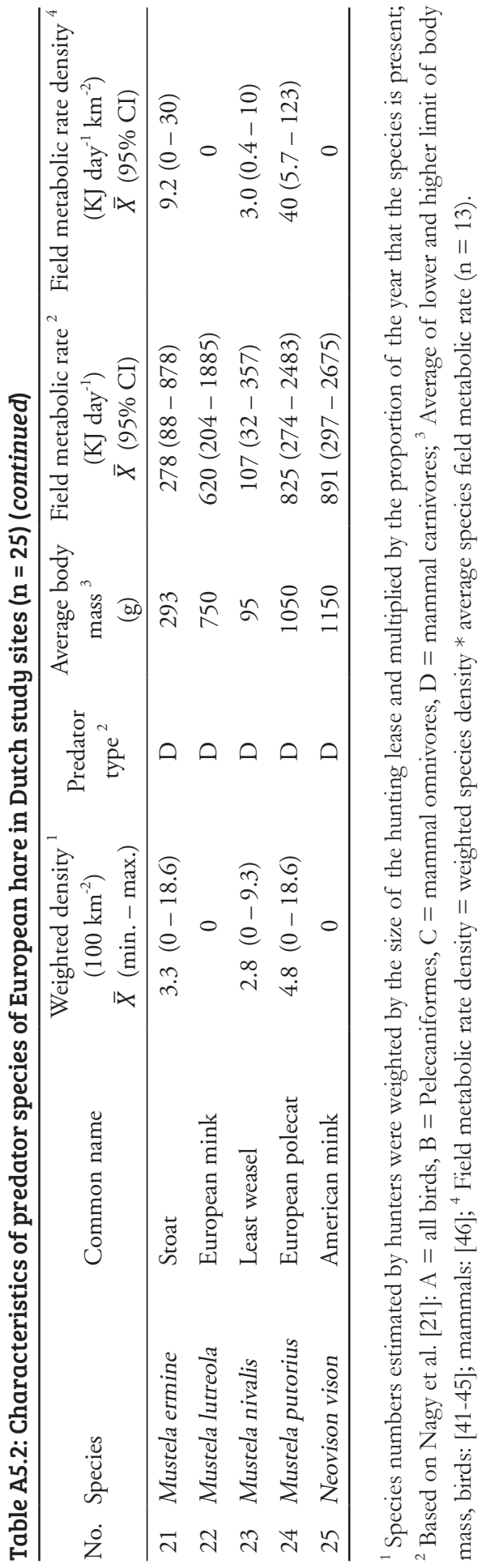


Table A5.3: An overview of European hare body measurements $(\overline{\mathrm{X}} \pm S D)$ per sex and age class, shot by hunters in the Netherlands.

\begin{tabular}{|c|c|c|c|c|c|c|c|c|}
\hline Sex & & Ma & & & & Fem & & \\
\hline Age class ${ }^{1}$ & Sub adult & $\mathrm{n}$ & Adult & $\mathrm{n}$ & Sub adult & $\mathrm{n}$ & Adult & $\mathrm{n}$ \\
\hline Body weight (g) & $3250 \pm 502$ & 13 & $3663 \pm 276$ & 22 & $3175 \pm 559$ & 17 & $3593 \pm 420$ & 19 \\
\hline Adrenal weight (avg.)(mg) & $280 \pm 52.8$ & 13 & $301 \pm 77.0$ & 22 & $291 \pm 67.2$ & 17 & $364 \pm 102$ & 18 \\
\hline Eye-lens weight (avg.) $(\mathrm{mg})^{2}$ & $197 \pm 43.8$ & 13 & $327 \pm 30.2$ & 22 & $201 \pm 34.4$ & 18 & $325 \pm 31.0$ & 19 \\
\hline Heart weight $(\mathrm{g})$ & $35.8 \pm 6.55$ & 10 & $42.6 \pm 5.65$ & 20 & $34.7 \pm 8.12$ & 14 & $45.0 \pm 6.35$ & 18 \\
\hline Kidney weight (avg.)(g) & $8.61 \pm 0.87$ & 10 & $9.66 \pm 0.80$ & 20 & $8.58 \pm 1.03$ & 12 & $10.2 \pm 0.96$ & 17 \\
\hline Liver weight (g) & $87.9 \pm 16.7$ & 11 & $90.1 \pm 19.7$ & 20 & $93.2 \pm 23.0$ & 15 & $96.3 \pm 14.3$ & 18 \\
\hline Zygomatic width (mm) & $43.3 \pm 3.27$ & 13 & $46.5 \pm 2.15$ & 22 & $43.8 \pm 3.09$ & 17 & $45.5 \pm 3.55$ & 19 \\
\hline Hind foot length (avg.) (cm) & $14.4 \pm 0.43$ & 13 & $14.6 \pm 0.71$ & 22 & $14.3 \pm 0.58$ & 17 & $14.6 \pm 0.48$ & 19 \\
\hline Ear length (avg.)(cm) & $9.68 \pm 0.49$ & 13 & $9.72 \pm 0.56$ & 22 & $9.46 \pm 0.54$ & 17 & $9.67 \pm 0.52$ & 19 \\
\hline
\end{tabular}

Placental scars

$\mathrm{n}=0$

abnormalities

$\mathrm{n}>0$ \& no abnormalities

$\begin{array}{cccc}0 \pm 0 & 2 & 0 \pm 0 & 1 \\ 0 \pm 0 & 1 & 0.5 \pm 0.71 & 2 \\ 10.0 \pm 5.20 & 3 & 10.9 \pm 3.31 & 15\end{array}$

${ }^{1}$ Age class is based on eye lens weight adult $>270 \mathrm{mg}$ : Adult: $>1$ year, Sub adult $\leq 1$ year old.

${ }^{2}$ Protocol by Broekhuizen and Maaskamp (1979), eye-lens weight air dried at $80^{\circ} \mathrm{C}$ for 6 days. 
Table A5.4: Results of the linear mixed models of the effect of species distribution (SD), hare density (HD), hunting effort (EFFORT), species body mass (BM), proportion of the year a predator was resident (PRO), and the size of the hunting lease (AREA) on the density of predators estimated by hunters $(n=10)$.

\begin{tabular}{|c|c|c|c|c|c|c|}
\hline No. & Model type $^{1}$ & & $d f$ & $\mathrm{AICc}^{2}$ & $\triangle \mathrm{AICc}$ & $\mathrm{w}_{\mathrm{i}}^{3}$ \\
\hline 1 & $\mathrm{SD}+\mathrm{AREA}+\mathrm{EFFORT}$ & & 6 & 210.8 & 0.0 & 0.66 \\
\hline 2 & SD + AREA & & 5 & 212.1 & 1.4 & 0.34 \\
\hline 3 & $\mathrm{SD}+\mathrm{HD}$ & & 5 & 226.5 & 15.8 & $<0.01$ \\
\hline 4 & SD & & 4 & 226.7 & 15.9 & $<0.01$ \\
\hline 5 & AREA + EFFORT + PRO & & 6 & 260.4 & 49.6 & $<0.01$ \\
\hline 6 & AREA + PRO & & 5 & 262.4 & 51.6 & $<0.01$ \\
\hline 7 & AREA + EFFORT & & 5 & 262.6 & 51.9 & $<0.01$ \\
\hline 8 & AREA & & 4 & 264.7 & 53.9 & $<0.01$ \\
\hline 9 & $\mathrm{HD}+\mathrm{PRO}$ & & 5 & 279.3 & 68.5 & $<0.01$ \\
\hline 10 & $\mathrm{HD}$ & & 4 & 281.6 & 70.8 & $<0.01$ \\
\hline 11 & PRO & & 4 & 282.4 & 71.6 & $<0.01$ \\
\hline 12 & Intercept & & 3 & 284.7 & 73.9 & $<0.01$ \\
\hline & Variables & Estimate $\left(\beta \pm \widehat{\mathrm{SE}}_{\beta}\right)^{4}$ & $\underset{5}{Z} Z_{\text {value }}$ & \multicolumn{2}{|c|}{$2.5 \%-97.5 \%$ C.I. } & $\mathrm{W}_{\mathrm{p}}{ }^{6}$ \\
\hline & AREA & $-0.18 \pm 0.04$ & $4.3^{*}$ & \multicolumn{2}{|c|}{$-0.27-1.00$} & 1.0 \\
\hline & EFFORT & $0.08 \pm 0.04$ & 1.9 & \multicolumn{2}{|c|}{$-0.004-0.16$} & 0.66 \\
\hline & HD & $0.06 \pm 0.04$ & 1.5 & \multicolumn{2}{|c|}{$-0.02-0.15$} & $<0.01$ \\
\hline & PRO & $0.28 \pm 0.13$ & $2.2^{*}$ & \multicolumn{2}{|c|}{$0.03-0.53$} & $<0.01$ \\
\hline & SD & $0.54 \pm 0.07$ & $7.8^{*}$ & \multicolumn{2}{|c|}{$0.40-0.67$} & 1.0 \\
\hline & Intercept & $|<0.01| \pm 0.05$ & $<0.1$ & \multicolumn{2}{|c|}{$-0.11-0.11$} & 1.0 \\
\hline
\end{tabular}

${ }^{1}$ Parsimonious models only; Models are based on estimate of 25 predator species by 10 hunters; Random factor: species; Models that included body mass of predators did not appear in parsimonious models and were left out; ${ }^{2}$ AICc $=$ Aikaike Information Criterion corrected for small sample size; ${ }^{3}$ wi $=$ Akaike weight or relative weight of each model; ${ }^{4}$ Estimates based on full-model conditional averaging of parsimonious models only; parameters are standardized by 2 SD (Gelman, 2008), ${ }^{5} *=$ $95 \%$ confidence intervals do not include zero; ${ }^{6} \mathrm{Wp}$ : Akaike predictor weight. 



\section{CHAPTER 6}

\section{General discussion}

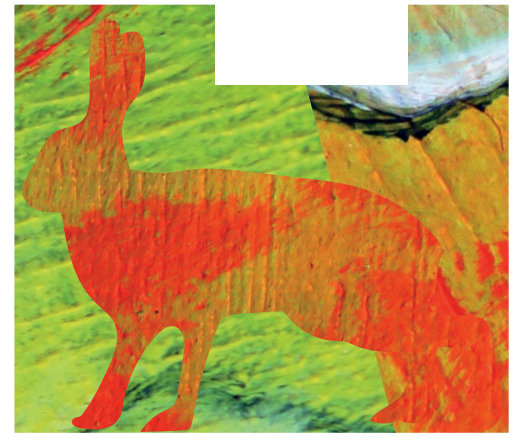





\section{INTRODUCTION}

The relative importance and the role of predators in ecosystems for prey is still subject of debate (Smith et al., 2003; Dobson, 2014). An understanding of the relative strength of the mechanisms (e.g., bottom-up and top-down) that modulate prey species behaviour, distribution and abundance will also contribute to an improved understanding of the responses of prey species to the changes in human-dominated landscapes. The objective of the thesis was to investigate the effects of predation risk and habitat characteristics on European hare. This will help us to understand the responses of prey to the changes in the north-west European landscape. In this thesis I report on: (1) The effect of elevated predation risk on the movement of the European hare in patches of different vegetation structure, (2) The effect of predators, vegetation structure and interspecific competitors (henceforth "competitors") on the space use of the European hare, (3) The effect of predators on the foraging behaviour of the European hare, relative to the effect of food quality, quantity and competitors, and (4) The effect of chronic exposure to predation risk of multiple predators on the body condition and reproductive output of the European hare.

In chapter 2, the reactive movement response of European hare to elevated predation risk was best explained by a model that included the interaction between predation risk and vegetation structure. On a short timescale (i.e., several hours), a strong immediate response was found in open habitat with low cover. However, on a longer timescale (i.e., 24 hours), the effect of the treatment was synchronized with the daily rhythm of the hare. The distance covered between resting and foraging grounds was negatively affected by elevated predation risk, while use of less risky (often low-quality) vegetation during resting and foraging was favoured.

In chapter 3 I found that the response of prey to the space use by predators (i.e., foxes), space use by fellow prey, and habitat characteristics was different for both prey species investigated (i.e., hare and rabbit). The level of activity by rabbits, which make use of a refuge for escape (i.e., central-place forager), was positively correlated with the level of activity by predators over time. However, space use of rabbits was uncorrelated to space use by predators. The level of activity by hares, which do not make use of a refuge (i.e., free-ranging), was uncorrelated with the level of activity by predators over time. However, space use of hares was correlated with space use by predators. Predator absence possibly led to avoidance behaviour, while predator presence promoted coexistence between the two sympatric prey species. Competition for predator free space may have reversed 
the relationship between the space use by predators and habitat characteristics on the space use by prey.

In chapter 4 I showed that hares spent a higher proportion of time in patches of food with a lower quality (i.e., a higher percentage of fibres). During days that predators were more active, hares spent a higher proportion of time in low-risk patches, and in patches with low-quality food and low-quantity food. Overall, habitat characteristics more strongly affected hare space use than the activity of predators. The activity of smaller competitors was not related to space use. During days that smaller competitors were more active, hares spent a higher proportion of time foraging in patches of low-quality food. Overall, habitat characteristics more strongly affected hare foraging time than the activity of predators, and the activity of smaller competitors was least important.

In chapter $5 \mathrm{I}$ found that predation risk was negatively correlated with the number of placental scars and the 'health' component of body condition of European hare (i.e., weight of liver, kidney, heart and body). Especially the presence of predators with a large average body mass and a large estimated density (i.e., grey heron, domestic or feral cat, red fox, and Eurasian buzzard), result in a high year-round predation risk for the hares.

\section{Relative importance and role of predation risk for hares}

'The 'top-down vs bottom-up' dichotomy is far too simplistic to handle the complex interactions' between predators and prey species (Hugie \& Dill, 1994, p.163). The results in this thesis show that both bottom-up and top-down forces modulate prey species behaviour and distribution simultaneously. Generally, the relative importance of top-down and bottom-up processes varies between systems and within systems that vary in environmental heterogeneity (Hunter \& Price, 1992). Because of this, the relative importance of top-down and bottom-up processes should be discussed in an explicit framework that amongst others incorporates spatial scale (Gripenberg \& Roslin, 2007).

The relative effect size (measured by the standardized coefficient, i.e., beta) of predators, habitat characteristics and competitors in models explaining movement, space use, foraging behaviour, body condition and reproductive output of European hare from chapter two, three, four and five of this thesis are summarized in table 6.1. Space use on a small scale (i.e., the 'food patch' (sensu Owen-Smith et al., 2010), $\pm 10-25 \mathrm{~m}^{2}$ ) was strongly affected by an interaction between predators and habitat characteristics, strongly affected by an interaction between competitors and habitat characteristics, and moderately affected by habitat characteristics alone. Note, however, that I did not investigate the relative strength of forage 
quality and quantity in explaining space use on a small scale. Space use on a large scale (i.e., the 'daily range' (sensu Owen-Smith et al., 2010), $\pm 10-100$ ha), however, was moderately affected by habitat characteristics, weakly affected by predators and not affected by competitors. The time spent foraging was weak to strongly affected by habitat characteristics, and only weakly affected by competitors and predators. Finally, predation was moderately to strongly related to body condition and reproductive output at a very large scale (i.e., the 'home range' (sensu Owen-Smith et al., 2010), > 100 ha). Note however, that I did not investigate the relative strength of habitat characteristics and competitors in explaining the body condition and reproductive output of European hare.

Predators affected space use solely via interactions with other variables, whereas they affected foraging behaviour and body condition and reproductive output solely as a main effect (figure 6.1a-d). Vegetation structure and forage quality affected space use and foraging behaviour via main effects and interactions with other variables. Competitors and forage quantity solely affected space use and foraging behaviour via interactions with other variables.

Overall, vegetation structure seemed to be important in affecting space use at a large (daily) scale, and very important in affecting foraging behaviour, and space use on a small (patch) scale (table 6.2). Forage quality seemed important in affecting foraging behaviour, and very important in affecting space use at a large scale. Predators seemed slightly important in affecting foraging behaviour and space use at a large scale, and fairly important in affecting body condition and reproductive output at a very large (home range) scale, and space use at a small scale. Forage quantity seemed slightly important in affecting space use at a large scale, and important in affecting foraging behaviour. Finally, competitors seemed not important in affecting space use at a large scale, slightly important in affecting foraging behaviour, and fairly important in affecting space use at a small scale. 


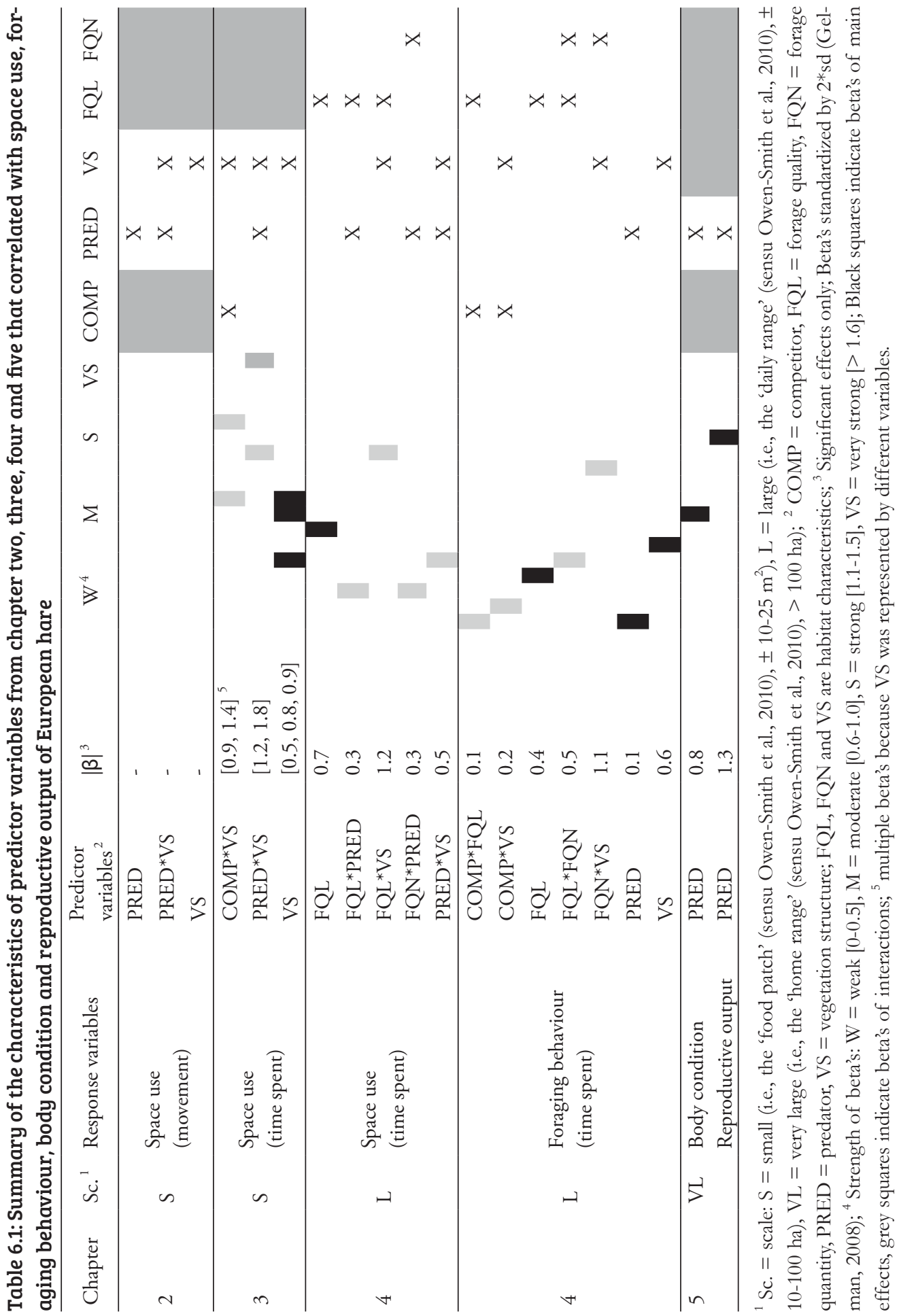


a

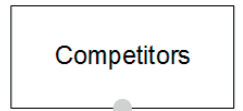

1.04

b

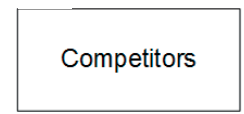

C
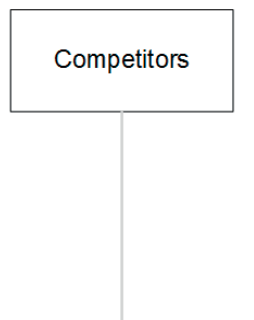

C
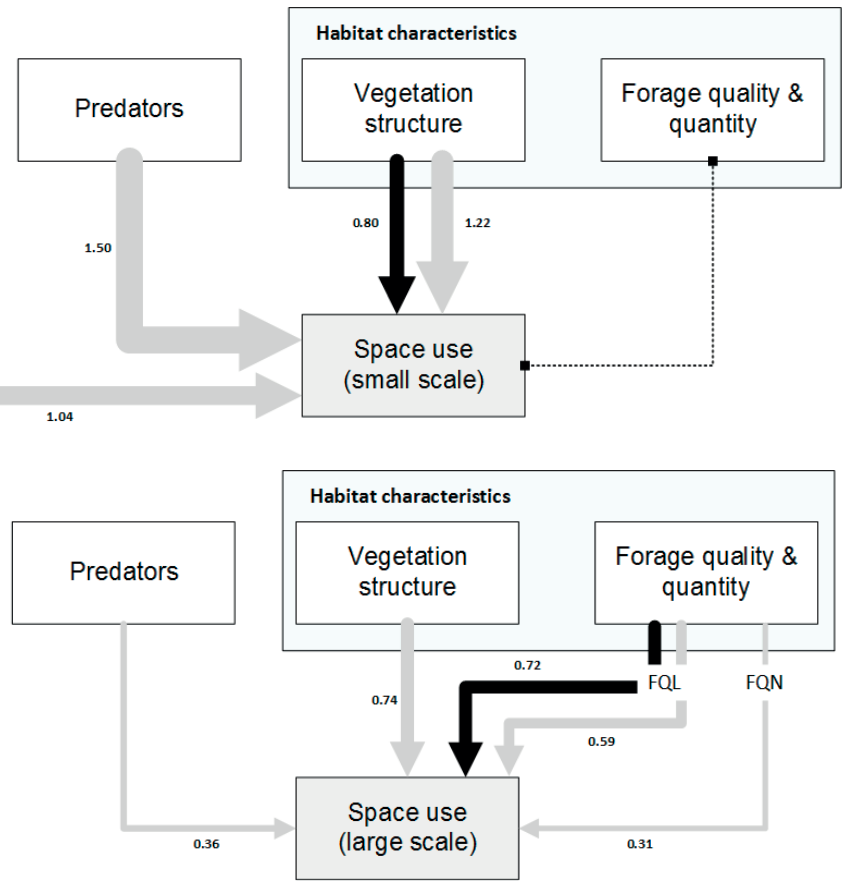

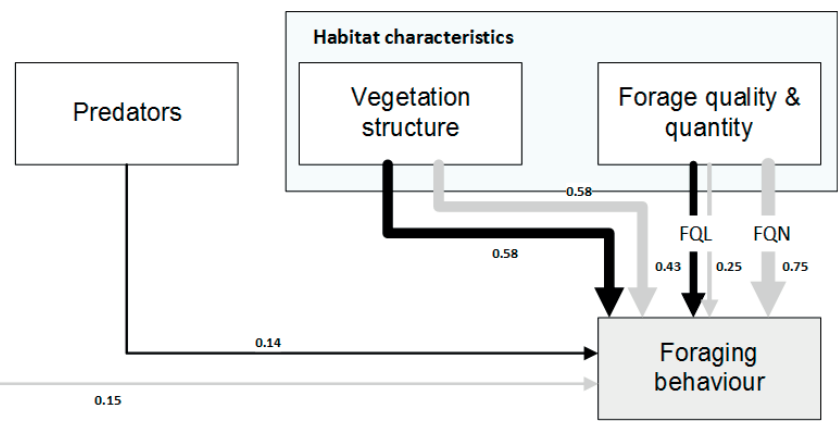

Interaction

Main effect

-.......... Relationship not investigated

d

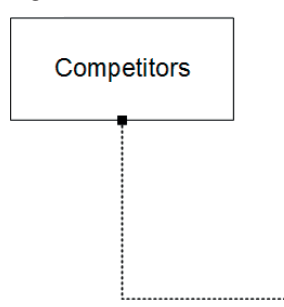

Body condition \&

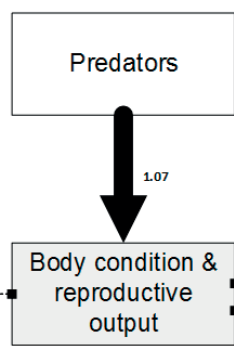

Habitat characteristics

Vegetation
structure

Forage quality \& quantity

Figure 6.1 
Figure 6.1: The weighted effect size of predators, habitat characteristics and competitors in models explaining: a) space use (small scale), b) space use (large scale), c) foraging behaviour, and d) body condition and reproductive output of European hare.

Thickness of arrows and numbers next to arrows represent effect size; Black arrows indicate beta's of main effects, grey arrows indicate beta's of interactions; Effect size of main effects are standardized beta's; Effect size of interactions are standardized beta's weighted by (standard error/beta) ${ }^{2}$ of predictor variable (Pullin \& Stewart, 2006). Sample size is number of times that the factor is part of a predictor variable for that response variable; Competitors (COMP), forage quality (FQL), forage quantity (FQN), predators (PRED) \& vegetation structure (VS); sample size is expressed as: factor(main effect, interaction); a) COMP $(0,2), \operatorname{PRED}(0,2)$ \& VS (3, 4), b) FQL (1, 2), FQN $(0,1), \operatorname{PRED}(0,3)$ \& VS $(0,2)$, c) COMP $(0,2)$, FQL $(1,2)$, FQN $(0,2)$, PRED $(1,0) \&$ VS $(1,2)$, d) PRED (2, 0); Scales of investigation (sensu Owen-Smith et al., 2010): Space use at a small scale ('food patch' : $\pm 10-25 \mathrm{~m}^{2}$ ), space use and foraging behaviour at a large scale ('daily range': $\pm 10-100 \mathrm{ha}$ ), and body condition and reproductive output at a very large scale ('home range': $>100 \mathrm{ha}$ ). (continued)

Table 6.2: Relative rank of predators, habitat characteristics and competitors in models explaining: space use, foraging behaviour, and body condition and reproductive output of European hare.

\begin{tabular}{|c|c|c|c|c|c|c|c|c|}
\hline Rank & $\begin{array}{c}0 \\
\text { not important }\end{array}$ & & $\begin{array}{c}1 \\
\text { slightly important }\end{array}$ & $\underset{\text { important }}{2}$ & $\begin{array}{c}3 \\
\text { fairly important }\end{array}$ & & $\begin{array}{c}4 \\
\text { very important }\end{array}$ & Scale \\
\hline Space use & & & & & $\begin{array}{l}\text { COMP } \\
\text { PRED }\end{array}$ & $<$ & VS & Food patch \\
\hline Space use & COMP & $<$ & $\begin{array}{l}\text { FQN } \\
\text { PRED }\end{array}$ & vs & & $<$ & FQL & Daily range \\
\hline $\begin{array}{l}\text { Foraging } \\
\text { behaviour }\end{array}$ & & & $\begin{array}{l}\text { COMP } \\
\text { PRED }\end{array}$ & $\begin{array}{l}\text { FQL } \\
\text { FQN }\end{array}$ & & $<$ & VS & Daily range \\
\hline $\begin{array}{l}\text { Body condition \& } \\
\text { reproductive output }\end{array}$ & & & & & PRED & & & Home range \\
\hline
\end{tabular}

$\mathrm{COMP}=$ competitors, $\mathrm{FQL}=$ forage quality, $\mathrm{FQN}=$ forage quantity, $\mathrm{PRED}=$ predators, $\mathrm{VS}=$ vegetation structure. Scores for factors are summed and ranked by strength of main effects and interactions. Main effects and interactions are equally weighted. Scores are summed for each main effect and interaction: 1 if effect size is between 0 and 0.5, 2 if effect size is between 0.5 and 1.0, and 3 if effect size greater than 1.0. Scales of investigation (sensu Owen-Smith et al., 2010): 'food patch' $\pm 10-25 \mathrm{~m}^{2}$, 'daily range' $\pm 10-100 \mathrm{ha}$, and 'home range' $>100$ ha.

\section{Implications of the relative importance and role of predation risk for prey}

'Few failures are as unforgiving as the failure to avoid a predator' (Lima \& Dill, 1990, p.619). Predator consumption (i.e., kills) affects prey species' demographics, whereas predator risk effects can modulate prey species' behaviour (Lima \& Dill, 1990; Ylönen et al., 1992), physiology (Creel \& Christianson, 2008) and reproduction (Krebs et al., 2001; Sheriff et al., 2009). Risk effects trigger prey defensive 
strategies that are costly, for example, in terms of reduced foraging efficiency or reproductive output. Besides, risk effects are at least as strong as the effects of direct consumption, also because risk effects affect prey populations via various pathways (Preisser et al., 2005; Zanette et al., 2014). In this discussion, I focus solely on the risk effects of predators.

Body size (and reserves) and food availability (Owen-Smith et al., 2010; Hopcraft et al., 2010; 2012) determine the response of herbivore prey towards the trade-off between food and predation risk. If food quality is high, the behaviour of small prey is expected to be stronger affected by predation risk than food (Hopcraft et al., 2010; 2012) because food quality is not limiting and smaller prey is often exposed to multiple predators (Sinclair et al., 2003). This is in contrast to large prey $(>150 \mathrm{~kg}$ ) that is affected stronger by food quantity than predation. If food quality is low, the behaviour of small prey is expected to be stronger affected by food quality than predation, whereas large prey is still affected stronger by food quantity than predation (Hopcraft et al., 2010; 2012). Food quality is important, because the metabolic rate of small prey is relatively high (Schmidt-Nielsen, 1990), and mass-specific nutritional requirements increase with declining body mass (Demment \& Van Soest, 1985). Besides, small herbivorous mammals are limited in their digestive efficiency, and need highly digestible food compared to large herbivores (Owen-Smith, 1988), which will cost them more time in searching for patches with high-quality food. The strength of bottom-up and top-down forces varies among sites, because of the variation in local conditions (chapter $3 \& 4$ ), regional processes and species-specific differences (Gripenberg \& Roslin, 2007), such as body size. Understanding the landscape context in this thesis is therefore important to assess the relative strength of the mechanisms that modulate prey behaviour (Hopcraft et al., 2010; 2012). In this discussion, I will consider the European hare as a small-sized herbivore $( \pm 1-10 \mathrm{~kg})$, which I have investigated in several productive coastal landscapes with high rainfall, high nutrient deposition (Ceulemans et al., 2011), and high quality forage.

Predators seemed to be less important in affecting space use and foraging behaviour of small-sized herbivore prey at the scale of the daily range ( $\pm 10-100 \mathrm{ha}$ ) (chapter 4). At this scale, small-sized herbivore prey selected habitat patches in the landscape based on habitat characteristics, in particular forage quality, which does not corroborate with the prediction by Hopcraft et al. (2010). Even though predators must be avoided, food is the primary driver (Sinclair \& Krebs, 2002) affecting small-sized prey behaviour at the scale of the daily range. It is not uncommon for prey to compensate behaviourally for losses, for example in foraging, due to 
risk effects (Luttbeg et al., 2003). But eventually prey must forage relative to the associated time scale or starvation will force prey to focus on food acquisition, while exposing themselves to higher levels of predation risk (i.e., the "Stalingrad effect") (Brown et al., 1997). Relative to large herbivores, the higher metabolic rate and lower body reserves of small-sized herbivores will reduce the timeframe before starvation sets in, and thus leaves less time to avoid predation risk with the associated foraging costs at the scale of the daily range. Additionally, several other factors could explain the relative importance of predators in the selection of habitat patches of small-sized herbivores. The presence of additional prey biomass will affect the diversity and density of predators (Carbone \& Gittleman, 2002). Changes in prey and predator community composition will alter various relationships between prey and its predators (Duffy et al., 2007), and thus also the risk perceived by prey. Effects of predators on prey species depend on hunting mode, habitat domain (Schmitz, 2007), interactions with other predators (VanceChalcraft \& Soluk, 2005; Lampropoulos et al., 2013), and resource specificity (Duffy et al., 2007) that can vary during the course of the season. Besides on energy and nutrients (i.e., nitrogen), small-sized herbivores make decisions based on trade-offs between intake rate, plant chemistry and digestibility (Wilmshurst et al., 2000; Shipley, 2007; Camp et al., 2015). Hopcraft et al. (2012) only considered nitrogen concentrations in their analysis, whereas in this study fibre content and possibly plant chemistry were also important in affecting prey space use of hare (chapter 4). Especially during winter time, hare can forage on grasses that contain a higher concentration of fibres but with lower levels of lignin compared to dicotyledonous species (Iason \& Van Wieren, 1999). Overall, the trade-off between food and predation at the scale of the 'daily range' thus depends on the community composition, species, state of the prey and predators, prey physiology, multiple characteristics of plant resources, as well as the season under investigation. Nevertheless, at the scale of the daily range, bottom-up modulation of prey space use and foraging behaviour seemed much more important than modulation by top-down processes.

In this thesis I also showed that predators seemed fairly important in affecting small-sized herbivore prey space use at the scale of the food patch $\left( \pm 10-25 \mathrm{~m}^{2}\right)$ (chapter $2 \& 3$ ). Inside the daily range, prey species select locations at the scale of the food patch, based on the predation risk experienced by prey. At the associated time scale of the food patch, small-sized herbivores do not have a lot of time to respond to the immediate threat of a predator. Prey species primarily used habitat characteristics (i.e., vegetation structure) to assess the predation risk of a location (chapter $2 \& 3$ ), followed by cues indicating the presence and activity of 
predators and competitors (i.e., fellow prey) (chapter 3). Habitat characteristics such as vegetation structure are correlated with visibility and predator encounter rates (Gorini et al., 2012). According to models of patch use under predation risk, animals either reduce behaviours that attract predators, such as moving, or they reduce behaviours that increase the encounter rate with predators (Brown, 1999). For example, an increase in predation risk can encourage prey to avoid the attention of the predator (Ydenberg \& Dill, 1986; Broom \& Ruxton, 2005) by moving to a safer location with more cover. The spatial distribution of the habitat characteristics may affect habitat-dependent escape tactics (Heithaus et al., 2009). Habitats with high cover reduce the detection distance of approaching predators and facilitate crypsis, whereas habitats with low cover enable prey to see approaching predators and facilitate flight (Hewson, 1977; Focardi \& Rizzotto, 1999). Besides, high cover benefits stealth dependent predators (i.e., ambush or stalker), whereas low cover benefits coursers. Prey anti-predator behaviour in a habitat with specific characteristics thus depends on the characteristics of the local predator, such as the hunting mode (Creel, 2011). Therefore, my results showed that prey space use was only affected by the interaction between predators and habitat characteristics. Nevertheless, at the scale of the food patch, bottomup modulation of prey space use seemed more important than modulation by top-down processes, or processes from the same trophic level (i.e., competitors).

At the scale of the home range ( $>100 \mathrm{ha}$ ), predators seemed fairly important in affecting body condition and reproductive output (i.e., reproductive fecundity sensu Zanette et al., 2014) of small-sized herbivore prey (chapter 5). These results corroborate with studies that have demonstrated a strong relationship between predation risk and the reproductive output of mammals (Monclús et al., 2011; Sheriff et al., 2009, 2010, 2011), and birds (Eggers et al., 2006; Zanette et al., 2011). It has been suggested earlier that the effects of predators on reproductive survival (sensu Zanette et al., 2014) could be compensated by mechanisms such as a higher reproductive output (Panek et al., 2006), however, this seems unlikely because prey species' reproductive output seems strongly negatively affected by predators as well. This means that predators affect prey species population dynamics via various pathways (Zanette et al., 2014). The impact of multiple predators on prey reproductive output, and the pathways followed, depends on the range in body sizes of the predators relative to the prey and their resource specificity (Sinclair et al., 2003). Communities that consist solely of small or specialist predators affect only a few similar-sized prey species (Norrdahl \& Korpimäki, 2000). Nevertheless, at the scale of the home range, top-down modulation of prey reproductive output seemed fairly important. 
In this thesis, predators seemed to be fairly important on the scale of the food patch, while slightly important on the scale of the daily range. Prey can show both reactive and predictive responses to perceived predation risk. Reactive behavioural responses are based on current knowledge and result in an immediate response at the scale of the food patch. Predictive responses are based on previous knowledge and do not result in an immediate response (Valeix et al., 2009), but are for example expressed during habitat selection at the scale of the daily range. The physiological response of prey to predation risk can affect the behavioural response, morphological response and individual fitness of prey (Sheriff \& Thaler, 2014). My conclusion is that the relative importance of predators for the behavioural response of prey (i.e., space use and foraging behaviour) is negatively correlated with the scale of the processes, probably linked to the age of the cues prey species collect about predators (e.g., Bytheway et al., 2013). In contrast, prey physiological responses that affect prey fitness (i.e., body condition and reproductive output) can scale up to population and community dynamics via alterations in developmental pathways (Sheriff \& Thaler, 2014; Zanette et al., 2014). Therefore, the relative importance of predators for prey fitness is probably positively related with the scale of the processes.

\section{Implications of the increased impact of predators on the European hare in the north-west European landscape}

Insight into the effects of predation risk and habitat characteristics on the European hare, will help us to understand the responses of hares to the changes in the north-west European landscape. Because hares shift habitat use under the risk of predation (chapter $2,3 \& 4$ ), affecting their foraging efficiency (chapter 4), predator risk effects could lead to a behaviourally mediated trophic cascade (Schmitz et al., 2004). Ecosystems on land, especially with a small herbivore-toplant body-size ratio (Shurin \& Seabloom, 2005), and smaller predators (i.e., red fox) (DeLong et al., 2015), show weak trophic cascades. However, Kuijper and Bakker (2005), for example, showed that exclusion of European hares allowed the invasion of late successional plant species in a coastal habitat, with shifts in species composition being most pronounced in areas of low productivity. Top-down processes on the scale of the daily range caused hare to shift its habitat use from areas of low productivity (i.e., short vegetation) to areas of high productivity (i.e., tall vegetation) (chapter $3 \& 4$ ). In terrestrial systems of high productivity, herbivory increases plant diversity and fertilization reduces plant species diversity, whereas this is vice versa in systems of low productivity (Hillebrand et al., 2007). Despite the effect of fertilization (i.e., eutrophication), the increased impact of 
predators could thus have increased plant species diversity in systems of high and low productivity (see also Kuijper \& Bakker, 2005). Currently however, even systems that were low in productivity in the north-west European landscape 50 years ago are now eutrophicated (Ceulemans et al., 2011). Besides, foraging behaviour (i.e., herbivory) and space use of hares is more strongly affected by food availability than by predation. The increased impact of predators on other small-sized herbivorous mammals, however, could trigger a behaviourally mediated trophic cascade. Rabbits, for example, can prevent graminoid dominance (Ten Harkel \& Van der Meulen, 1996), can attain high densities (Holland, 1999), are relatively defenceless outside of their burrow, and trade safety inside their burrow against a reduced resource intake (Van der Stap et al., 2007). I would expect the increased impact of predators on European rabbits to have resulted in a trophic cascade, especially in systems with easily digested plants (Hall et al., 2007). Nevertheless, since the 1950s rabbit populations in north-west Europe were probably more strongly affected by myxomatosis (Provoost et al., 2011) and since 1984 by Rabbit Haemorrhagic Disease (RHD) (Frölich et al., 2002) than predation.

As a result of the homogenisation of the north-west European landscape, the increased impact of predators probably had a negative effect on the population trend of European hares. First, because predators affect prey species population dynamics via various pathways (Zanette et al., 2014), and second, because predation risk strongly affects hare reproductive output (chapter 5). A two times increase in predator field metabolic rate would reduce the number of placental scars of hares by about $20 \%$. Especially predator species with a large average body mass and a large estimated density, such as grey heron (Ardea cinerea), domestic or feral cat (Felis catus), European buzzard (Buteo buteo) and red fox (Vulpes vulpes) that contribute to an above average field metabolic rate density have increased in numbers during the time that hare population sizes decreased. For example, between 1979-2005 there was a 50\% increase in the percentage of Dutch households that own cats (RDA, 2006). European buzzard and grey heron breeding pairs have increased in the Netherlands by about $5000 \%$ and $300 \%$ respectively between 1901 and 2000 (Parlevliet, 2003). Between 1970-1998, red fox population numbers increased in West Germany (Knauer et al., 2010). Knauer et al. (2010), however, noted that that the changes in the agricultural landscapes probably more strongly affected hare population numbers. Nevertheless, this thesis showed that the effect of multiple predators should be taken into account to assess the effect of predators on hare population numbers, which has not been done by Knauer et al. (2010). 
So, what will happen to the population size of the European hare in the Netherlands in the near future? Recently, predator population sizes of grey heron (-23\% (2000-2016); NEM et al., 2017), domestic cat (-26\% (2005-2014); RDA, 2006; HAS Hogeschool \& faculteit Diergeneeskunde, 2015) and red fox (-12\% (1993-2015); NEM et al., 2015) have decreased. However, the European buzzard population size has increased even further between 2000-2016 (+36\%; NEM et al., 2017) (also note the strong recent increase in the population size of the great egret (Ardea alba)). At the same time, the population size of European hare in the Netherlands seems to have stabilized between 2000 and 2015 (NEM et al., 2015). Based on the recent decrease in population trends of some of the most important predators of the European hare, I would expect the population size of European hare in the Netherlands to slightly increase in the near future. However, this shall be separate from the current conversion of additional land to agricultural use and further agricultural intensification on existing productive land (Dauber \& Miyake, 2016) in the north-west European landscape. This will undoubtedly increase the loss in additional habitat diversity, structure and quality in the agricultural landscape even further (see e.g., Gamero et al., 2017). 




\section{Bibliography}



Apfelbach, R., Blanchard, C.D., Blanchard, R.J., Hayes, R.H. \& McGregor, I.S. (2005) The effects of predator odors in mammalian prey species: A review of field and laboratory studies. Neuroscience and Biobehavioural Reviews, 29: 1123-1144.

Arsenault, R. \& Owen-Smith, N. (2002) Facilitation versus competition in grazing herbivore assemblages. Oikos, 97(3): 313-318.

Ashby, K.R. (1972) Patterns of daily activity in mammals. Mammal Review, 1: 171-185.

Bakker, E.S., Reiffers, R.C., Olff, H. \& Gleichman, J.M. (2005) Experimental manipulation of predation risk and food quality: effect on grazing behaviour in a central-place foraging herbivore. Oecologia, 146: 157-167.

Bakker, E.S., Olff, H. \& Gleichman, J.M. (2009) Contrasting effects of large herbivore grazing on smaller herbivores. Basic and Applied Ecology, 10: 141-150.

Bal, D., Beije, H.M., Fellinger, N., Haveman, R., Van Opstal, A.J.F.M. \& Van Zadelhoff, F.J. (2001) Handboek Natuurdoeltypen. 2nd edition. Expertcentre LNV, Wageningen.

Barboza, P.S., Parker, K.L. \& Hume, I.D. (2009) Integrative wildlife nutrition. Springer-Verlag Berlin Heidelberg, pp 342.

Barnes, R.F.W. \& Tapper, S.C. (1986) Consequences of the myxomatosis epidemic in Britain's rabbit (Oryctolagus cuniculus L.) population on the numbers of brown hares (Lepus europaeus Pallas). Mammal Review, 16(3/4): 111-116.

Bednekoff, P.A. \& Lima, S.L. (1998) Re-examining safety in numbers: interactions between risk dilution and collective detection depend upon predator targeting behaviour. Proceedings of the Royal Society B, Biological Sciences, 265: 2021-2026.

Bedoya-Perez, M.A., Carthey, A.J.R., Mella, V.S.A., McArthur, C. \& Banks, P.B. (2013) practical guide to avoid giving up on giving-up densities. Behavioural Ecology and Sociobiology, 67: 1541-1553.

Bell, R.H.V. (1971) A grazing ecosystem in the Serengeti. Scientific American, 225: 86-93. Benjamini, Y. \& Hochberg, Y. (1995) Controlling the false discovery rate: A practical and powerful approach to multiple testing. Journal of the Royal Statistical Society. Series B, 57(1): 289-300.

Benton, T.G., Vickery, J.A. \& Wilson, J.D. (2003) Farmland biodiversity: is habitat heterogeneity the key? Trends in ecology and evolution, 18(4): 182-188.

Bian, J.H., Du, S.Y., Wu, Y., Cao, Y.F., Nie, X.H., He, H. \& You, Z.B. (2015) Maternal effects and population regulation: maternal density-induced reproduction suppression impairs offspring capacity in response to immediate environment in root voles Microtus oeconomus. Journal of Animal Ecology, 84, 326-336.

Bissett, C. \& Bernard, R.T.F. (2007) Habitat selection and feeding ecology of the cheetah (Acinonyx jubatus) in thicket vegetation: is the cheetah a savannah specialist? Journal of Zoology, 271: 310-317.

Bjørneraas, K., van Moorter, B., Rolandsen, C.M. \& Herfindal, I. (2010) Screening global positioning system location data for errors using animal movement characteristics. Journal of Wildlife Management, 74: 1361-1366.

Boele A., Hustings F., Koffijberg K., van Turnhout C. \& Plate C. (2008) Populatietrends van terrestrische wintervogels in 1980-2006: habitat, trekgedrag en verschillen tussen Hoog- en Laag Nederland. Limosa, 81: 50-61.

Bom, R.A., Bouten, W., Piersma, T., Oosterbeek, K. \& Van Gils, J.A. (2014) Optimizing acceleration-based ethograms: the use of variable-time versus fixed-time segmentation. Movement Ecology, 2(6): 1-8.

Bonsall, M.B. \& Hassell, M.P. (2000) Apparent competition structures ecological assemblages. Nature, 388: 371-373.

Bonsall, M.B., Bull, J.C., Pickup, N.J. \& Hassell, M.P. (2005) Indirect effects and spatial scaling affect the persistence of multispecies metapopulations. Proceedings of the Royal Society B, Biological Sciences, 272: 1465-1471. 
Boonstra, R., Hik, D., Singleton, G.R. \& Tinnikov, A. (1998) The impact of predator-induced stress on the snowshoe hare cycle. Ecological Monographs, 79(5): 371-394.

Bray, Y., Champely, S. \& Soyez, D. (2002) Age determination in leverets of European hare Lepus europaeus based on body measurements. Wildlife Biology, 8: 31-39.

Bray, Y., Marboutin, É., Péroux, R. \& Ferron, J. (2003) reliability of stained placental-scar counts in European hares. Wildlife Society Bulletin, 31(1): 237-246.

Bresinski, W. (1983) The effect of some habitat factors on the spatial distribution of a hare population during the winter. Acta Theriologica, 28-29: 435-441.

Broekhuis, F., Cozzi, G., Valeix, M., McNutt, J.W. \& Macdonald, D.W. (2013) Risk avoidance in sympatric large carnivores: reactive or predictive? Journal of Animal Ecology, 82: 1098-1105.

Broekhuizen, S. (1982) Hazen in Nederland. Studies on the population ecology of hares in the Netherlands. Research Institute for Nature Management, Arnhem, The Netherlands.

Broekhuizen, S. \& Maaskamp, F. (1979) Age determination in the European hare (Lepus europaeus Pallas) in the Netherlands. Zeitschrift fur Säugetierkunde, 44: 162-175.

Broom, M. \& Ruxton, G.D. (2005) You can run-or you can hide: optimal strategies for cryptic prey against pursuit predators. Behavioural Ecology, 16: 534-540.

Brown, J.H., Gillooly, J.F., Allen, A.P., Van Savage, M. \& West, G.B. (2004) Toward a metabolic theory of ecology. Ecology, 85(7): 1771-1789.

Brown, J.S. (1999) Vigilance, patch use and habitat selection: foraging under predation risk. Evolutionary Ecology Research, 1: 49-71.

Brown, J.S. \& Kotler, B.P. (2004) Hazardous duty pay and the foraging cost of predation. Ecology Letters, 7: 999-1014.

Brown, J.S., Kotler, B.P. \& Mitchell, W.A. (1997) Competition between birds and mammals: a comparison of giving- up densities between crested larks and gerbils. Evolution \& Ecology, 11: 757-771.

Burnham, K.P. \& Anderson, D.R. (2002) Model Selection and Multimodel Inference: A Practical informationTheoretic Approach. 2nd ed. New York, NY: Springer.

Bytheway, J.P., Carthey, A.J.R. \& Banks, P.B. (2013) Risk vs. reward: how predators and prey respond to aging olfactory cues. Behavioural Ecology and Sociobiology, 67(5): 715-725.

Camp, M.J., Shipley, L.A., Johnson, T.R., Forbey, J.S. \& Rachlow, J.L. (2015) Modeling trade-offs between plant fiber and toxins: a framework for quantifying risks perceived by foraging herbivores. Ecology, 96(12): 3292-3302.

Caravaggi, A., Montgomery, W.I. \& Reid, N. (2015) Range expansion and comparative habitat use of insular, congeneric lagomorphs: invasive European hares Lepus europaeus and endemic Irish hares Lepus timidus hibernicus. Biological Invasions, 17: 687-698.

Carbone, C. \& Gittleman, J.L. (2002) A common rule for the scaling of carnivore density. Science, 295(5563): 2273-2276.

Careau, V., Thomas, D., Humphries, M.M. \& Réale, D. (2008) Energy metabolism and animal personality. Oikos, 117(5): 641-653.

Carlson, S.M., Edeline, E., Vøllestad, L.A., Haugen, T.O., Winfield, I.J., Fletcher, J.M., James, J.B. \& Stenseth, N.C. (2007) Four decades of opposing natural and human-induced artificial selection acting on Windermere pike (Esox Lucius). Ecology Letters, 10: 512-521.

Ceulemans, T., Merckx, R., Hens, M. \& Honnay, O. (2011) A trait-based analysis of the role of phosphorous vs. nitrogen enrichment in plant species loss across North-west European grasslands. Journal of Applied Ecology, 48: 1155-1163.

Chaneton, E.J. \& Bonsall, M.B. (2000) Enemy-mediated apparent competition: empirical patterns and the evidence. Oikos, 88: 380-394. 
Chesson, P. (2000) General theory of competitive coexistence in spatially-varying environments. Theoretical Population Biology, 58: 211-237.

Chesson, P. \& Kuang, J.J. (2008) The interaction between predation and competition. Nature, 456: 235-238.

Ciuti, S., Northrup, J.M., Muhly, T.B., Simi, S., Musiani, M., Pitt, J.A. \& Boyce, M.S. (2012) Effects of humans on behaviour of wildlife exceed those of natural predators in a landscape of fear. PLoS ONE, 7(11): e50611. DOI 10.1371/journal.pone.0050611.

Clark, L.S., Cowan, D.F. \& Pfeiffer, D.C. (2006) Morphological changes in the Atlantic bottlenose dolphin (Tursiops truncates) adrenal gland associated with chronic stress. Journal of Comparative Pathology, 135: 208-216.

Clinchy, M., Zanette, Y.L., Charlier, T.D., Newman, A.E.M., Schmidt, K.L., Boonstra, R. \& Soma, K.K. (2011) Multiple measures elucidate glucocorticoid responses to environmental variation in predation threat. Oecologia, 166: 607-614.

Coltman, D.W., O’Donoghue, P., Jorgenson, J.T., Hogg, J.T., Strobeck, C. \& Festa-Blanchet, M. (2003) Undesirable evolutionary consequences of trophy hunting. Nature, 426: 655-658.

Cooper, Jr. W.E. \& Sherbrooke, W.C. (2013) Effects of recent movement, starting distance and other risk factors on escape behaviour by two phrynosomatid lizards. Behaviour, 150: 447-469.

Crawley, M.J. (2007) The R book. John Wiley \& Sons, Ltd, Chichester, pp 1076.

Creel, S. (2011) Towards a predictive theory of risk effects: hypotheses for prey attributes and compensatory mortality. Ecology, 92(12):2190-2195.

Creel., S. \& Christianson, D. (2008) Relationships between direct predation and risk effects. Trends in Ecology and Evolution, 23(4):194-201.

Creel, S., Christianson, D., Liley, S. \& Winnie-Jr, J.A. (2007) Predation risk affects reproductive physiology and demography of elk. Science, New Series, 315(5814): 960.

Creel, S., Winnie, J.A., Christianson, D. \& Liley, S. (2008) Time and space in general models of antipredator response: tests with wolves and elk. Animal Behaviour, 76: 1139-1146.

Cromsigt, J.P.G.M., Kuijper, D.J.P., Adam, M., Beschta, R.L., Churski, M., Eycott, A., Kerley, G.I.H., Mysterud, A., Schmidt, K. \& West, K. (2013) Hunting for fear: innovating management of human-wildlife conflicts. Journal of Applied Ecology, 50: 544-549.

Crowell, M.M., Shipley, L.A., Camp, M.J., Rachlow, J.L., Forbey, J.S. \& Johnson, T.R. (2016) Selection of food patches by sympatric herbivores in response to concealment and distance from a refuge. Ecology and Evolution, 6(9): 2865-2876. doi: 10.1002/ece3.1940.

Darimont, C.T., Carlson, S.M., Kinnison, M.T., Paquet, P.C., Reimchen, T.E. \& Wilmers, C.C. (2009) Human predators outpace other agents of trait change in the wild. PNAS, 106(3): 952-954.

Dauber, J. \& Miyake, S. (2016) To integrate or to segregate food crop and energy crop cultivation at the landscape scale? Perspectives on biodiversity conservation in agriculture in Europe. Energy, Sustainability and Society, 6:25. DOI 10.1186/s13705-016-0089-5.

Davidson, R.K., Romig, T., Jenkins, E., Tryland, M. \& Robertson, L.J. (2012) The impact of globalisation on the distribution of Echinococcus multilocularis. Trends in Parasitology, 28(6): 239-247.

De Bruyn, L., Scheirs, J. \& Verhagen, R. (2002) Nutrient stress, host plant quality and herbivore performance of a leaf-mining fly on grass. Oecologia, 130: 594-599.

DeCesare, N.J., Hebblewhite, M., Robinson, H.S. \& Musiani, M. (2010) Endangered, apparently: the role of apparent competition in endangered species conservation. Animal Conservation, 13: 353-362.

Del Hoyo, J., Elliot, A. \& Christie, D.A. (eds) (2009) Bush-shrikes to Old World Sparrows, Volume 14, Handbook of the Birds of the World. Lynx Edicions, Barcelona.

Del Hoyo, J., Elliot, A. \& Sargatal, J. (eds) (1999) Barn-owls to Hummingbirds, Volume 5, Handbook of the Birds of the World. Lynx Edicions, Barcelona. 
Del Hoyo, J., Elliot, A. \& Sargatal, J. (eds) (1996) Hoatzin to Auks, Volume 3, Handbook of the Birds of the World. Lynx Edicions, Barcelona.

Del Hoyo, J., Elliot, A. \& Sargatal, J. (eds) (1994) New World Vultures to Guineafowl, Volume 2, Handbook of the Birds of the World. Lynx Edicions, Barcelona.

Del Hoyo, J., Elliot, A. \& Sargatal, J. (eds) (1992) Ostrich to Ducks, Volume 1, Handbook of the Birds of the World. Lynx Edicions, Barcelona.

DeLong, J.P., Gilbert, B., Shurin, J.B., Savage, V.M., Barton, B.T., Clements, C.E., Dell, A.I., Greig, H.S., Harley, C.D.G., Kratina, P., McCann, K.S., Tunney, T.D., Vasseur, D.A. \& O’Connor, M.I. (2015) The body size dependence of trophic cascades. The American Naturalist, 185(3): 354-366.

Demment, M.W. \& Van Soest, P.J. (1985) A nutritional explanation for body-size patterns of ruminant and non- ruminant herbivores. The American Naturalist, 125: 641-672.

Dennis, T.E. \& Shah, S.F. (2012) Assessing acute effects of trapping, handling, and tagging on the behaviour of wildlife using GPS telemetry: a case study of the common brushtail possum. Journal of Applied Animal Welfare Science, 15: 189-207.

DiStefano, C., Zhu, M. \& Mîndrilă, D. (2009) Understanding and using factor scores: considerations for the applied researcher. Practical Assessment, Research \& Evaluation, 14(20): 1-11.

Dobson, A.P. (2014) Yellowstone wolves and the forces that structure natural systems. PLoS Biology, 12(12): e1002025. doi:10.1371/journal.pbio.1002025.

Duffy, J.E., Cardinale, B.J., France, K.E., McIntyre, P.B., Thébault, E. \& Loreau, M. (2007) The functional role of biodiversity in ecosystems: incorporating trophic complexity. Ecology Letters, 10: 522-538.

Ebling, F.J.P. \& Barrett, P. (2008) The regulation of seasonal changes in food intake and body weight. Journal of Neroendocrinology, 20: 827-833.

Eccard, J.A. \& Liesenjohann, T. (2014) The importance of predation risk and missed opportunity costs for context-dependent foraging patterns. PloS One, 9(5): e94107.

Eccard, J.A., Pusenius, J., Sundell, J., Halle, S. \& Ylönen, H. (2008) Foraging patterns of voles at heterogeneous avian and uniform mustelid predation risk. Oecologia, 157: 725-734.

Edwards, P.J., Fletcher, M.R. \& Berny, P. (2000) Review of the factors affecting the decline of the European Brown hare, Lepus europaeus (Pallas, 1778) and the use of wildlife incident data to evaluate the significance of paraquat. Agriculture, Ecosystems and Environment, 79: 95-103.

Eggers, S., Griesser, M., Nystrand, M. \& Ekman, J. (2006) Predation risk induces changes in nest-site selection and clutch size in the Siberian Jay. Proceedings of the Royal Society B, 273: 701-706.

Everts, F.H., Pranger, D.P., Tolman, M.E. \& De Vries, N.P.J. (2008) Vegetatiekartering deelgebieden EgmondBakkum 2007. Report: 653 EGG, EGG consult, Groningen, the Netherlands.

Everts, F.H., Pranger, D.P., Tolman, M.E. \& De Vries, N.P.J. (2009) Vegetatiekartering deelgebied Castricum 2008. Reportnumber: 739 EGG, EGG consult, Groningen, the Netherlands.

FAO (2010) 2000 World Census of Agriculture. Main Results and Metadata by Country (1996-2005) FAO Statistical development series 12. FAO, Rome, Italy.

Fernández, A., Soriguer, R., Carro, F. \& Castién, E. (2010) Relation between physical condition and reproductive activity in a population of Iberian hares, Lepus granatensis in northern Iberian peninsula, Folia Zoologica, 59(1): 1-8.

Ferrari, M.C.O., Sih, A. \& Chivers, D.P. (2009) The paradox of risk allocation: a review and prospectus. Animal Behaviour, 78: 579-585.

Ferretti, F., Corazza, M., Campana, I., Pietrocini, V., Brunetti, C., Scornavacca, D. \& Lovari, S. (2015) Competition between wild herbivores: reintroduced red deer and Apennine chamois. Behavioural Ecology, 26(2): 550-559. 
Filion, F.L. (1980) Human surveys in wildlife management. In: Schemnitz, S.D. (ed) Wildlife Techniques Manual, 4th edition. The Wildlife Society: Washington, DC, pp 441-453.

Flux, J.E.C. (1993) Relative effect of cats, myxomatosis, traditional control, or competitors in removing rabbits from islands. New Zealand Journal of Zoology, 20: 13-18.

Flux, J.E.C. (2008) A review of competition between rabbits (Oryctolagus cuniculus) and hares (Lepus europaeus). In: Alves, P.C., Ferrand, N. \& Hackländer, K. (2008) Lagomorph biology: Evolution, ecology and conservation. Springer-Verlag Berlin Heidelberg, Germany.

Focardi, S., Aragno. P., Montanaro, P. \& Riga, F. (2006) Inter-specific competition from fallow deer Dama dama reduces habitat quality for the Italian roe deer Capreolus capreolus italicus. Ecography, 29: 407-417.

Focardi, S. \& Rizzotto, M. (1999) Optimal strategies and complexity: a theoretical analysis of the anti-predatory behaviour of the hare. Bulletin of Mathematical Biology, 61: 829-848.

Fortin, D., Beyer, H., Boyce, M.S., Smith, D.W., Duchesne, T. \& Mao, J.S. (2005) Wolves influence elk movements: behaviour shapes a trophic cascade in Yellowstone National Park. Ecology, 86(5): 1320-1330.

Frey, S.N. \& Conover, M.R. (2006) Habitat use by meso-predators in a corridor environment. Journal of Wildlife Management, 70(4): 1111-1118.

Friar, J.L., Nielsen, S.E., Merrill, E.H., Lele, S.R., Boyce, M.S., Munro, R.H.M., Stenhouse, G.B. \& Beyer, H.L. (2004) Removing GPS collar bias in habitat selection studies. Journal of Applied Ecology, 41: 201-212.

Frid, A., \& Dill, L.M. (2002) Human-caused disturbance stimuli as a form of predation risk. Conservation Ecology, 6: 11-27.

Frölich, K., Thiede, S., Kozikowski, T. \& Jakob, W. (2002) A review of mutual transmission of important infectious diseases between livestock and wildlife in Europe. Annals of the New York Academy of Sciences, 969: 4-13.

Gamero, A., Brotons, L., Brunner, A., Foppen, R., Fornasari, L., Gregory, R.D., Herrando, S., Hořák, D., Jiguet, F., Kmecl, P., Lehikoinen, A., Lindström, Å., Paquet, J-Y., Reif, J., Sirkiä, P.M., Škorpilová, J., Van Strien, A., Szép, T., Telenský, T., Teufelbauer, N., Trautmann, S., Van Turnhout, C.A.M., Vermouzek, Z., Vikstrøm, T. \& Voř́šek, P. (2017) Tracking progress toward EU biodiversity strategy targets: EU policy effects in preserving its common farmland birds. Conservation Letters, 10(4): 395-402.

Geiger, F., Bengtsson, J., Berendse, F., Weisser, W.W., Emmerson, M., Morales, M.B., Ceryngier, P., Liira, J., Tscharntke, T., Winqvist, C., Eggers, S., Bommarco, R., Pärt, T., Bretagnolle, V., Plantegenest, M., Clement, L.W., Dennis, C., Palmer, C., Oñate, J., Guerrero, I., Hawro, V., Aavik, T., Thies, C., Flohre, A., Hänke, S., Fischer, C., Goedhart, P.W. \& Inchausti, P. (2009) Persistent negative effects of pesticides on biodiversity and biological control potential on European farmland. Basic and Applied Ecology, doi:10.1016/j.baae.2009.12.001

Gelman, A. (2008) Scaling regression inputs by dividing by two standard deviations. Statistics in Medicine, 27: 2865-2873.

Gerritsmann, H., Stalder, G.L., Seilern-Moy, K., Knauer, F. \& Walzer, C. (2012) Comparison of S(+)-ketamine and ketamine, with medetomidine, for field anaesthesia in the European brown hare (Lepus europaeus). Veterinary Anaesthesia and Analgesia, 39: 511-519.

Gorini, L., Linnell, J.D.C., May, R., Panzacchi, M., Boitani, L., Odden, M. \& Nilsen, E.B. (2012). Habitat heterogeneity and mammalian predator-prey interactions. Mammal Review, 42: 55-77.

Grand, T.C. \& Dill, L.M. (1999a) The effect of group size on the foraging behaviour of juvenile coho salmon: reduction of predation risk or increased competition? Animal Behaviour, 58: 443-451.

Grand, T.C. \& Dill, L.M. (1999b) Predation risk, unequal competitors and the ideal free distribution. Evolutionary Ecology Research, 1: 389-409.

Griffin, K.A., Hebblewhite, M., Robinson, H.S., Zager, P., Barber-Meyer, S.M., Christianson, D., Creel, S., Harris, N.C., Hurley, M.A., Jackson, D.H., Johnson, B.K., Myers, W.L., Raithel, J.D., Schlegel, M., Smith, 
B.L., White, C. \& White, P.J. (2011) Neonatal mortality of elk driven by climate, predator phenology and predator community composition. Journal of Animal Ecology, 80: 1246-1257.

Griffin, J.N., Toscano, B.J., Griffen, B.D. \& Silliman, B.R. (2015) Does relative abundance modify multiple predator effects? Basic and Applied Ecology, 16: 641-651.

Gripenberg, S. \& Roslin, T. (2007) Up or down in space? Uniting the bottom-up versus top-down paradigm and spatial ecology. Oikos, 116: 181-188.

Grueber, C.E., Nakagawa, S., Laws, R.J. \& Jamieson, I.G. (2011) Multimodel inference in ecology and evolution: challenges and solutions. Journal of Evolutionary Biology, 24: 699-711.

Hackländer, K., Frisch, C., Klansek, E., Steineck, T. \& Ruf, T. (2001) Die fruchtbarkeit weiblicher feldhasen (Lepus europaeus) aus revieren mit unterschiedlicher populationsdichte. Zeitschrift fur Jagdwissenschaft, 47: 100-110.

Hackländer, K., Zeitlhofer, C., Ceulemans, T. \& Suchentrunk, F. (2011) Continentality affects body condition and size but not yearly reproductive output in female European hares (Lepus europaeus). Mammalian Biology, 76: 662-664.

Hall, S.R., Shurin, J.B., Diehl, S. \& Nisbet, R.M. (2007) Food quality, nutrient limitation of secondary production, and the strength of trophic cascades. Oikos, 116: 1128-1143.

Halliday, W.D. \& Morris, D.W. (2013) Safety from predators or competitors? Interference competition leads to apparent predation risk. Journal of Mammalogy, 94(6): 1380-1392.

Halpin, M.A. \& Bissonette, J.A. (1988) Influence of snow depth on prey availability and habitat use by red fox. Canadian Journal of Zoology, 66: 587-592.

Harder, J.D. \& Kirkpatrick, R.L. (1994) Physiological methods in wildlife research. In: Bookhout, T.A. (ed) Research and management techniques for wildlife and habitats, 5th edition. The Wildlife Society, Bethesda, Maryland, pp 275-306.

HAS Hogeschool \& faculteit Diergeneeskunde (2015) Feiten \& cijfers Gezelschapsdierensector 2015. Ministerie van Economische Zaken, Den Haag

Hauzy, C., Tully, T., Spataro, T., Paul, G. \& Arditi, R. (2010) Spatial heterogeneity and functional response: an experiment in microcosms with varying obstacle densities. Oecologia, 163: 625-636.

Hawlena, D., Schmitz, O.J. (2010) Physiological stress as a fundamental mechanism linking predation to ecosystem functioning. The American Naturalist, 176(5): 537-556.

Heithaus, M.R., Wirsing, A.J., Burkholder, D., Thomson, J. \& Dill, L.M. (2009) Towards a predictive framework for predator risk effects: the interaction of landscape features and prey escape tactics. Journal of Animal Ecology, 78: 556-562.

Hernández, L. \& Laundré, J.W. (2005) Foraging in the 'landscape of fear' and its implications for habitat use and diet quality of elk Cervus elaphus and bison Bison bison. Wildlife Biology, 11(3): 215-220.

Heuermann, N., Van Langevelde, F., Van Wieren, S.E. \& Prins, H.H.T. (2011) Increased searching and handling effort in tall swards lead to a Type IV functional response in small grazing herbivores. Oecologia, 166: 659-669.

Hewson, R. (1977) Food selection by brown hares (Lepus capensis) on cereal and turnip crops in north-east Scotland. Journal of Applied Ecology, 14: 779-785.

Hillebrand, H., Gruner, D.S., Borer, E.T., Bracken, M.E.S., Cleland, E.E., Elser, J.J., Harpole, W.S., Ngai, J.T., Seabloom, E.W., Shurin, J.B. \& Smith, J.E. (2007) Consumer versus resource control of producer diversity depends on ecosystem type and producer community structure. PNAS, 104(26): 10904-10909.

Hodges, K.E. \& Sinclair, A.R.E. (2005) Browse site selection by snowshoe hares: effects of food supply and predation risk. Canadian Journal of Zoology, 83: 280-292.

Hoeksma, W. (1950) De familie Lepus. Deventer, the Netherlands: N.V. Uitgevers-maatschappij Æ. E. Kluwer. Holland, M.K. (1999) Fertility control in wild populations of animals. Journal of Andrology, 20(5): 579-585. 
Holt, R.D. (2009) Predation and community organization. In: Levin, S.A. (ed.) The Princeton guide to ecology. Princeton University press, Princeton, USA.

Holt, R.D. \& Kotler, B.P. (1987) Short-term apparent competition. The American Naturalist, 130(3): 412-430.

Holt, R.D. \& Lawton, J.H. (1994) The ecological consequences of shared natural enemies. Annual Review of Ecology, Evolution, and Systematics, 25: 495-520.

Homolka, M. (1987) The diet of brown hare (Lepus europaeus) in central Bohemia. Folia zoologica, 36(2): 103-110.

Hopcraft, J.G.C., Olff, H., \& Sinclair, A.R.E. (2010) Herbivores, resources and risks: alternating regulation along primary environmental gradients in savannas. Trends in Ecology and Evolution, 25, 119-128.

Hopcraft, J.G.C., Anderson, T.M., Pérez-Vila, S., Mayemba, E. \& Olff, H. (2012) Body size and the division of niche space: food and predation differentially shape the distribution of Serengeti grazers. Journal of Animal Ecology, 81: 201-213.

Huber, J. (2004) Heer Lampe: de Europese haas in Nederland. Koninklijke Vermande, Den Haag.

Hugie, D.M. \& Dill, L.M. (1994) Fish and game: a game theoretic approach to habitat selection by predators and prey. Journal of Fish Biology, 45: 151-169.

Hunter, M. \& Price, P. (1992) Playing chutes and ladders: heterogeneity and the relative roles of bottom-up and top-down forces in natural communities. Ecology, 73: 724-732.

Hustings, F. \& Vergeer, J-W. (eds.) (2002) Atlas van de Nederlandse broedvogels 1998-2000: verspreiding, aantallen, verandering. Nederlandse fauna 5. Nationaal Natuurhistorisch Museum Naturalis, Leiden. KNNV Uitgeverij, Utrecht.

Hutchings, M.R. \& Harris, S. (1996) The current status of the Brown hare (Lepus europaeus) in Britain. Joint Nature Conservation Committee, Birmingham, UK.

Iason, G., Van Wieren, S.E. (1999) Digestive and ingestive adaptations of mammalian herbivores to low-quality forage. In: Olff, H., Brown, V.K. \& Drent, R.H. Herbivores: Between Plants and Predators. Oxford, Blackwell Science, p.337-370.

IUCN (2017) IUCN Red List of threatened species: Lepus europaeus. Version 2017.2. www.iucnredlist.org [21-11-2017]

Jansen, P.A., Forrester, T.D. \& McShea, W.J. (2014) Protocol for camera-trap surveys of mammals at CTFSForestGEOsites. Smithsonian Tropical Research Institute, center for tropical forest science, Ancon, Panamá.

Johnson, H.E., Hebblewhite, M., Stephenson, T.R., German, D.W., Pierce, B.M. \& Bleich, V.C. (2013) Evaluating apparent competition in limiting the recovery of an endangered ungulate. Oecologia, 171: 295-307.

Johnson, A.R., Wiens, J.A., Milne, B.T. \& Crist, T.O. (1992) Animal movements and population dynamics in heterogeneous landscapes. Landscape Ecology, 7: 63-75.

Jonker, R.M., Eichhorn, G., Van Langevelde, F. \& Bauer, S. (2010) Predation danger can explain changes in timing of migration: the case of the barnacle goose. PLoS ONE, 5(6): e11369. doi:10.1371/journal. pone.0011369.

Kaiser, H.F. (1960) The application of electronic computers to factor analysis. Educational and Psychological Measurement, 20: 141-151.

Katona, K., Bíró, Z., Hahn, I., Kertész, M. \& Altbäcker, V. (2004) Competition between European hare and European rabbit in a lowland area, Hungary: a long-term ecological study in the period of rabbit extinction. Folia Zoologica, 53(3): 255-268.

Kauffman, M.J., Brodie, J.F. \& Jules, E.S. (2010) Are wolves saving Yellowstone's aspen? A landscape-level test of a behaviourally mediated trophic cascade. Ecology, 91(9): 2742-2755.

Kauffman, M.J., Varley, M., Smith, D.W., Stahler, D.R., MacNulty, D.R. \& Boyce, M.S. (2007) Landscape heterogeneity shapes predation in a newly restored predator-prey system. Ecology Letters, 10: 690-700. 


\section{Bibliography}

Kiener, T.V. \& Zaitsev, V.A. (2010) Range structure in the red fox (Vulpes vulpes L.) in the forest zone of Eastern Europe. Contemporary Problems of Ecology, 3(1): 119-126.

Knauer, F., Küchenhoff, H. \& Pilz, S. (2010) A statistical analysis of the relationship between red fox Vulpes vulpes and its prey species (grey partridge Perdix perdix, brown hare Lepus europaeus and rabbit Oryctolagus cuniculus) in Western Germany from 1958 to 1998. Wildlife Biology, 16: 56-65.

Kooijman, A.M., Dopheide, J.C.R., Sevink, J., Takken, I. \& Verstraten, J.M. (1998) Nutrient limitations and their implications on the effects of atmorspheric deposition in coastal dunes; lime-poor and lime-rich sites in the Netherlands. Journal of Ecology, 86: 511-526.

Koops, M.A. (2004) Reliability and the value of information. Animal Behaviour, 67: 103-111.

Kotler, B. \& Blaustein, L. (1995) Titrating food and safety in a heterogeneous environment: when are the safe and risky patches of equal value? Oikos, 74: 251-258

Krebs, C.J. (2009) Ecology: the experimental analysis of distribution and abundance. 6th ed. Benjamin Cummings, San Francisco, USA. 655 pp.

Krebs, C.J., Boonstra, R., Boutin, S., Sinclair, A.R.E. (2001) What drives the 10-year cycle of snowshoe hares? Bioscience, 51(1): 25-35.

Kuijper, D.J.P. \& Bakker, J.P. (2005) Top-down control of small herbivores on saltmarsh vegetation along a productivity gradient. Ecology, 86: 914-923.

Kuijper, D.P.J. \& Bakker, J.P. (2008) Unpreferred plants affect patch choice and spatial distribution of Brown hares. Acta Oecologica, 34: 339-344.

Kuijper, D.P.J., Beek, P., Van Wieren, S.E. \& Bakker, J.P. (2008) Time-scale effects in the interaction between a large and a small herbivore. Basic and Applied Ecology, 9: 126-134.

Kuijper, D.P.J., Bubnicki, J.W., Churski, M., Mols, B. \& Van Hooft, P. (2015) Context dependence of risk effects: wolves and tree logs create patches of fear in an old-growth forest. Behavioural Ecology, 26(6): 1558-1568.

Kuijper, D.P.J., de Kleine, C., Churski, M., van Hooft, P., Bubnicki, J. \& Jędrzejewska, B. (2013) Landscape of fear in Europe: wolves affect spatial patterns of ungulate browsing in Białowieża Primeval Forest, Poland. Ecography, 36: 1263-1275.

Kuijper, D.P.J., van Wieren, S.E. \& Bakker, J.P. (2004) Digestive strategies in two sympatrically occurring lagomorphs. Journal of Zoology London, 264: 1-8.

Kuijper, D.P.J., Verwijmeren, M., Churski, M., Zbyryt, A., Schmidt, K., Jędrzejewska, B. \& Smit, C. (2014) What cues do ungulates use to assess predation risk in dense temperate forests? PloS ONE, 9: 1-12.

Kunst, P.J.G., van der Wal, R. \& van Wieren, S. (2001) Home ranges of brown hares in a natural salt marsh: comparisons with agricultural systems. Acta Theriologica, 46: 287-294.

Lamarque, F., Barrat, J. \& Moutou, F. (1996) Principal diagnoses for determining causes of mortality in the European Brown hare (Lepus europaeus) found dead in France between 1986 and 1994. Gibier Faune Sauvage, 13(1):53-72.

Lamoot, L. (2004) Foraging behaviour and habitat use of large herbivores in a coastal dune landscape. Ph.D thesis, University of Ghent, Belgium.

Lampropoulos, P.D., Perdikis, D.Ch. \& Fantinou, A.A. (2013) Are multiple predator effects directed by prey availability? Basic and Applied Ecology, 14: 605-613.

Lange, R., Twisk, P., Van Winden, A. \& Van Diepenbeek, A. (2003) Zoogdieren van West-Europa., 2e druk. ed. KNNV Uitgeverij, Utrecht.

Laundré, J.W. (2010) Behavioural response races, predator-prey shell games, ecology of fear, and patch use of pumas and their ungulate prey. Ecology, 91(10): 2995-3007.

Laundré, J.W., Hernández, L. \& Altendorf, K.B. (2001) Wolves, elk, and bison: re-establishing the "landscape of fear" in Yellowstone National Park, U.S.A. Canadian Journal of Zoology, 79: 1401-1409. 
Laundré, J.W., Hernández, L. \& Ripple, W.J. (2010) The landscape of fear: Ecological implications of being afraid. The Open Ecology Journal, 3: 1-7.

Lenth, B.E., Knight, R.L. \& Brennan, M.E. (2008) The effects of dogs on wildlife communities. Natural Areas Journal, 28: 218-227.

Lima, S.L. (1992) Strong preferences for apparently dangerous habitats? A consequence of differential escape from predators. Oikos, 64: 597-600.

Lima, S.L. (1998) Stress and decision making under the risk of predation: recent developments from behavioural, reproductive and ecological perspectives. Advances in the Study of Behaviour, 27: 215-290.

Lima, S.L. (2002) Putting predators back into behavioural predator-prey interactions. TRENDS in Ecology \& Evolution, 17(2): 70-75.

Lima, S.L. \& Bednekoff, P.A. (1999) Temporal variation in danger drives antipredator behaviour: The predation risk allocation hypothesis. The American Naturalist, 153(6): 649-659.

Lima, S.L. \& Dill, L.M. (1990) Behavioural decisions made under the risk of predation: a review and prospectus. Canadian Journal of Zoology, 68: 619-640.

Lipták T. (1958) On the combination of independent tests. Publications of the Mathematical Institute of the Hungarian Academy of Sciences, 3: 171-196.

Lundström-Gilliéron, C. \& Schlaepfer, R. (2003) Hare abundance as an indicator for urbanization and intensification of agriculture in Western Europe. Ecological Modelling, 168: 283-301.

Luttbeg, B., Rowe, L. \& Mangel, M. (2003) Prey state and experimental design affect relative size of trait- and density-mediated indirect effects. Ecology, 84: 1140-1150.

Marboutin, E., Bray, Y., Péroux, R., Mauvy, B. \& Lartiges, A. (2003) Population dynamics in European hare: breeding parameters and sustainable harvest rates. Journal of Applied Ecology, 40: 580-591.

Møller, A.P. (2008) Flight distance and population trends in European breeding birds. Behavioural Ecology, 19: 1095-1102.

Monclús, R., Palomares, F., Tablado, Z., Martínez-Fontúrbel, A. \& Palme, R. (2009) Testing the threat-sensitive predator avoidance hypothesis: physiological responses and predator pressure in wild rabbits. Oecologia, 158(4): 615-623.

Monclús, R., Tiulim, J. \& Blumstein, D.T. (2011) Older mothers follow conservative strategies under predator pressure: the adaptive role of maternal glucocorticoids in yellow-bellied marmots. Hormones and Behaviour, 60: 660-665.

Morris, D.W. (2002) Toward an ecological synthesis: a case for habitat selection. Oecologia, 136: 1-13.

Morris,D.W. (2009) Apparent predation risk: tests of habitat selection theory reveal unexpected effects of competition. Evolutionary Ecology Research, 11: 209-225.

Mulder, J.L. (2005) Vossenonderzoek in de duinstreek van 1979 tot 2000. VZZ report 2005.72. Zoogdiervereniging VZZ, Arnhem, the Netherlands.

Nagy, K.A., Girard, I.A. \& Brown, T.K. (1999) Energetics of free-ranging mammals, reptiles, and birds. Annual Review of Nutrition, 19: 247-277.

Nagy, K.A. (2005) Review: Field metabolic rate and body size. The Journal of Experimental Biology, 208: 1621-1625.

Nathan, R., Spiegel, O., Fortmann-Roe, S., Harel, R., Wikelski, M. \& Getz, W.M. (2012) Using tri-axial acceleration data to identify behavioural modes of free-ranging animals: general concepts and tools illustrated for griffon vultures. The Journal of experimental biology, 215(6): 986-996.

NDFF (2015) NDFF Dutch mammal species distribution maps. ttp://verspreidingsatlas.nl/zoogdieren. [accessed: 22 October 2017].

NEM (2009) Aantalsontwikkeling dagactieve zoogdieren, 1994-2008. www.milieuennatuurcompendium.nl. VZZ, Arnhem, SOVON, Driebergen and CBS, Den Haag. 
NEM, CBS \& Zoogdiervereniging (2015) Trend red fox 1993-2015. Zoogdiervereniging, Nijmegen.

NEM, SOVON \& CBS (2017) Broedvogeltrends 1990-2016. Sovon Vogelonderzoek Nederland, Nijmegen.

Neumann, F., Schai-Braun, S., Weber, D. \& Amrhein, V. (2011) European hares select resting places for providing cover. Hystrix, the Italian Journal of Mammalogy, 22(2): 291-299.

Norbury, G. (2001) Conserving dryland lizards by reducing predator-mediated apparent competition and direct competition with introduced rabbits. Journal of Applied Ecology, 38: 1350-1361.

Norrdahl, K. \& Korpimäki, E. (2000) Do predators limit the abundance of alternative prey? Experiments with vole-eating avian and mammalian predators. Oikos, 91(3): 528-540.

Olesen, C.R. \& Asferg, T. (2006) Assessing potential causes for the population decline of European Brown hare in the agricultural landscape of Europe: a review of the current knowledge. NERI Technical Report No.600. National Environmental Research Institute, Ministry of the Environment, Copenhagen.

Oliver, M., Luque-Larena, J.J. \& Lambin, X. (2009) Do rabbits eat voles? Apparent competition, habitat heterogeneity and large-scale coexistence under mink predation. Ecology Letters, 12: 1201-1209.

Owen-Smith, N. (1988) Megaherbivores: The influence of very large body size on ecology. Cambridge University Press, Cambridge, UK.

Owen-Smith, N., Fryxell, J.M. \& Merrill, E.H. (2010) Foraging theory upscaled: the behavioural ecology of herbivore movement. Philosophical Transactions of the Royal Society B, 365: 2267-2278.

Paci, G., Ferretti, M. \& Bagliacca, M. (2012) Reducing visual stimulations in European hares (Lepus europaeus Pallas) captured for translocation. Italian Journal of Animal Science, 11: 275-278.

Padding, P.I. \& Royle, J.A. (2012) Assessment of bias in US waterfowl harvest estimates. Wildlife Research, 39(4): 336-342.

Panek, M., Kamieniarz, R. \& Bresiński, W. (2006) The effect of experimental removal of red foxes Vulpes vulpes on spring density of brown hares Lepus europaeus in western Poland. Acta Theriologica, 51(2): 187-193.

Parlevliet, J. (2003) Broedvogels in Nederland in de 20 eeuw. Limosa, 76: 141-156.

Pays, O., Blanchard, P., Valeix, M., Chamaillé-Jammes, S., Duncan, P., Périquet, S., Lombard, M., Ncube, G., Tarakini, T., Makuwe, E. \& Fritz, H. (2012) Detecting predators and locating competitors while foraging: an experimental study of a medium-sized herbivore in an African savanna. Oecologia, 169: 419-430.

Persson, L. (1985) Asymmetrical competition: are larger animals competitively superior? American Naturalist, 126: 261-266.

Peters, R.H. (1983) The ecological implications of body size. Cambridge University Press, Cambridge, UK.

Petrovan, S.O., Ward, A.I. \& Wheeler, P.M. (2013) Habitat selection guiding agri-environment schemes for a farmland specialist, the brown hare. Animal Conservation, 16: 344-352. Potts, J.R., Harris, S. \& Giuggioli, L. (2012) Territorial dynamics and stable home range formation for central place foragers. PLoS ONE 7(3): e34033. doi:10.1371/journal.pone.0034033

Pranger, D.P. \& Tolman, M.E. (2012) Toelichting bij de vegetatiekartering Schiermonnikoog 2010. Delft, the Netherlands: Rijkswaterstaat.

Preisser, E.L., Bolnick, D.I. \& Benard, M. (2005) Scared to death? The effects of intimidation and consumption in predator-prey interactions. Ecology, 86(2):501-509.

Prevedello, J.A., Forero-Medina, G. \& Vieira, M.V. (2010) Movement behaviour within and beyond perceptual ranges in three small mammals: effects of matrix type and body mass. Journal of Animal Ecology, 79: 1315-1323.

Prins, H.H.T. \& Olff, H. (1998) Species-richness of African grazer assemblages: Towards a functional explanation. In: Newbery, D.M., Prins, H.T.T. \& Brown, N.D. (Eds.) Dynamics of tropical communities. 37th Symposium of the British Ecological Society. 
Proffitt, K.M., Grigg, J.L., Hamlin, K.L. \& Garrott, R.A. (2009) Contrasting effects of wolves and human hunters on elk behavioural responses to predation risk. Journal of Wildlife Management, 73: 345-356.

Provoost, S., Laurence, M., Jones, M. \& Edmondson, S.E. (2011) Changes in landscape and vegetation of coastal dunes in northwest Europe: a review. Journal of Coastal Conservation, 15: 207-226.

Pullin, A.S. \& Stewart, G.B. (2006) Guidelines for systematic review in conservation and environmental management. Conservation Biology, 20(6): 1647-1656.

RDA (2006) Gedeelde zorg: feiten \& cijfers. Raad voor Dierenaangelegenheden. Den Haag.

Resheff, Y. S., Rotics, S., Harel, R., Spiegel, O. \& Nathan, R. (2014) AcceleRater: a web application for supervised learning of behavioural modes from acceleration measurements. Movement Ecology, 2(1): 25.

Reynolds, J.C. \& Tapper, S.C. (1995) Predation by foxes Vulpes vulpes on brown hares Lepus europaeus in central southern England, and its potential impact on annual population growth. Wildlife Biology, 1: 145-158.

Ripple, W.J., Larsen, E.J., Renkin, R.A. \& Smith, D.W. (2001) Trophic cascades among wolves, elk and aspen on Yellowstone National Park's northern range. Biological Conservation, 102: 227-234.

Ripple, W.J. \& Beschta, R.L. (2012) Trophic cascades in Yellowstone: the first 15 years after wolf reintroduction. Biological Conservation, 145: 205-213.

Robinson, R.A. \& Sutherland, W.J. (2002) Post-war changes in arable farming and biodiversity in Great Britain. Journal of Applied Ecology, 39:157-176.

Roedenbeck, I.A. \& Voser, P. (2008) Effects of roads on spatial distribution, abundance and mortality of Brown hare (Lepus europaeus) in Switzerland. European Journal of Wildlife Research, 54: 425-437.

Rowcliffe, J.M., Carbone, C., Jansen, P.A., Kays, R. \& Kranstauber, B. (2011) Quantifying the sensitivity of camera traps: an adapted distance sampling approach. Methods in Ecology and Evolution, 2: 464-476.

Riginos, C. \& Grace, J.B. (2008) Savanna tree density, herbivores, and the herbaceous community: Bottom-up vs. top-down effects. Ecology, 89: 2228-2238.

Rivrud, I.M., Sonkoly, K., Lehoczki, R., Csányi, S., Storvik, G.O. \& Mysterud, A. (2013) Hunter selection and long-term trend (1881-2008) of red deer trophy sizes in Hungary. Journal of Applied Ecology, 50: 168-180.

Robbins, C.T. (1993) Wildlife feeding and nutrition. $2^{\text {nd }}$ edition, Academic Press, New York, USA, pp 342.

Ruxton, G.D. \& Colegrave, N. (2011) Experimental design for the life sciences. 3rd ed. Oxford, U.K.: Oxford University Press.

Samelius, G. Andrén, H., Kjellander, P. \& Liberg, O. (2013) Habitat selection and risk of predation: re-colonization by lynx had limited impact on habitat selection by roe deer. PLoS ONE, 8(9): e75469. doi:10.1371/ journal.pone.0075469.

Schai-Braun, S.C., Rödel, H.G. \& Hackländer, K. (2012) The influence of daylight regime on diurnal locomotor activity patterns of the European hare (Lepus europaeus) during summer. Mammalian Biology, 77: 434-440.

Schmidt, N.M., Asferg, T. \& Forchhammer, M.C. (2004) Long-term patterns in European Brown hare population dynamics in Denmark: effects of agriculture, predation and climate. BMC Ecology, 4:15.

Schmidt-Nielsen K. (ed.) (1990) Animal physiology: adaptation and environment. Cambridge University Press, Cambridge, UK.

Schmitz, O.J., Krivan, V. \& Ovadia, O. (2004) Trophic cascades: the primacy of trait-mediated indirect interactions. Ecology Letters, 7: 153-163.

Schmitz, O.J. (2007) Predator diversity and trophic interactions. Ecology, 88(10): 2415-2426.

Schneider, M.F. (2001) Habitat loss, fragmentation and predator impact: spatial implications for prey conservation. Journal of Applied Ecology, 38: 720-735.

Schoener, T.W. (1989) Food webs from the small to the large. Ecology, 70: 1559-1589. 
Sheriff, M.J., Krebs, C.J. \& Boonstra, R. (2011) From process to pattern: how fluctuating predation risk impacts the stress axis of snowshoe hares during the 10-year cycle. Oecologica, 166: 593-605.

Sheriff, M.J., Krebs, C.J. \& Boonstra, R. (2010) The ghosts of predators past: population cycles and the role of the maternal programming under fluctuating predation risk. Ecology, 91(10): 2983-2994.

Sheriff, M.J., Krebs, C.J., Boonstra, R. (2009) The sensitive hare: sublethal effects of predator stress on reproduction in snowshoe hares. Journal of Animal Ecology, 78: 1249-1258.

Sheriff, M.J. \& Thaler, J.S. (2014) Ecophysiological effects of predation risk; an integration across disciplines. Oecologia, 176: 607-611.

Shipley, L.A. (2007) The influence of bite size on foraging at larger spatial and temporal scales by mammalian herbivores. Oikos, 116: 1964-1974.

Shrader, A.M., Kerley, G.I.H., Brown, J.S. \& Kotler, B.P. (2012) Patch use in free-ranging goats: Does a large mammalian herbivore forage like other central place foragers? Ethology, 118: 967-974.

Shurin, J.B. \& Seabloom, E.W. (2005) The strength of trophic cascades across ecosystems: predictions from allometry and energetics. Journal of Animal Ecology, 74: 1029-1038.

Sih, A. (2005) Predator-prey space use as an emergent outcome of a behavioural response race. In: Barbosa, P. \& Castellanos, I. (eds.) The ecology of predator-prey interactions. Oxford University Press, Oxford, UK. Pp. 240-255.

Sih, A., Bell, A. \& Johnson, J.C. (2004) Behavioural syndromes: an ecological and evolutionary overview. TRENDS in Ecology and Evolution, 19(7): 372-378.

Sih, A., Englund, G., Wooster, D. (1998) Emergent impacts of multiple predators on prey. Trends in Ecology and Evolution, 13(9): 350-355.

Sinclair, A.R.E. (1985) Does interspecific competition or predation shape the African ungulate community. Journal of Animal Ecology, 54: 899-918.

Sinclair, A.R.E. \& Krebs, C.J. (2002) Complex numerical responses to top-down and bottom-up processes in vertebrate populations. Philosophical Transactions: Biological Sciences, 357(1425): 1221-1231.

Sinclair, A.R.E., Mduma, S., \& Brashares, J.S. (2003) Patterns of predation in a diverse predator-prey system. Nature, 425, 288-290.

Smith, A.P. \& Quin, D.G. (1996) Patterns and causes of extinction and decline in Australian Conilurine rodents. Biological Conservation, 77: 243-267.

Smith, A.T. \& Johnston, C.H. (2008) Lepus europaeus. In: IUCN (2009) IUCN Red List of threatened species. Version 2009.2. www.iucnredlist.org [26-11-2009].

Smith, D.W., Peterson, R.O. \& Houston, D.B. (2003) Yellowstone after wolves. BioScience, 53(4): 330-340.

Smith, R.K., Jennings, N.V. \& Harris, S. (2005) A quantitative analysis of the abundance and demography of European hares Lepus europaeus in relation to habitat type, intensity of agriculture and climate. Mammal Review, 35: 1-24.

Smith, S., Mang, T., De Bellocq, J.G., Schaschl, H., Zeitlhofer, C., Hackländer, K., Suchentrunk, F. (2010) Homozygosity at a class II MHC locus depresses female reproductive ability in European brown hares. Molecular Ecology, 19: 4131-4143.

SOVON (2017) Dutch bird species distribution maps. http://www.vogelatlas.nl/ [accessed: 22 October 2017]

Stankowich, T. \& Blumstein, D.T. (2005) Fear in animals: a meta-analysis and review of risk assessment. Proceedings of the Royal Society B, 272: 2627-2634.

Stephens, D. \& Krebs, J.R. (1986) Foraging theory. Princeton University Press, Princeton, USA.

Stott, P. (2003) Use of space by sympatric European hares (Lepus europaeus) and European rabbits (Oryctolagus cuniculus) in Australia. Mammalian Biology, 68: 317-327. 
Stott, P. (2007) Comparisons of digestive function between the European hare (Lepus europaeus) and the European rabbit (Oryctolagus cuniculus): mastication, gut passage, and digestibility. Mammalian Biology, 73: 276-286.

Stott, P. \& Harris, S. (2006) Demographics of the European hare (Lepus europaeus) in the Mediterranean climate zone of Australia. Mammalian Biology, 4: 214-226.

Strong, D.R. (1992) Are trophic cascades all wet? Differentiation and donor-control in speciose ecosystems. Ecology, 73(3): 747-754.

Szymanski, M.L., Johnson, M.A. \& Grovijahn, M. (2013) Effects of hunting pressure and collection method bias on body mass of drake mallards. Management and Conservation, 77(2): 235-242.

Tapper, S. (1992) Game heritage: an ecological review from shooting and gamekeeping records. Game Conservancy Ltd., Fordingbridge.

Tapper, S.C. \& Barnes, R.F.W. (1986) Influence of farming practice on the ecology of the brown hare (Lepus europaeus). Journal of Applied Ecology, 23: 39-52.

Tapper, S. \& Parsons, N. (1984) The changing status of the brown hare (Lepus capensis L) in Britain. Mammal Review, 14: 57-70.

Tapper S \& Yalden D (2010) The brown hare. The Mammal Society, Southampton, UK.

Ten Harkel, M.J. \& Van der Meulen, F. (1996) Impact of grazing and atmospheric nitrogen deposition on the vegetation of dry coastal dune grasslands. Journal of Vegetation Science, 7(3): 445-452.

Thaker, M., Vanak, A.T., Owen, C.R., Ogden, M.B., Niemann, S.M. \& Slotow R. (2011) Minimizing predation risk in a landscape of multiple predators: effects on the spatial distribution of African ungulates. Ecology, 92: 398-407.

Travers, M., Clinchy, M., Zanette, Y.L., Boonstra, R. \& Williams, T.D. (2010) Indirect predator effects on clutch size and the cost of egg production. Ecology Letters, 13: 980-988.

Trewhella, W.J., Harris, S. \& McAllister, F.E. (1988) Dispersal distance, home-range size and population density in the red fox (Vulpes vulpes): a quantitative analysis. Journal of Applied Ecology, 25(2): 423-434.

Tryjanowski, P., Sparks, T.H., Kamieniarz, R. \& Panek, M. (2009) The relationship between hunting methods and sex, age and body weight in a non-trophy animal, the red fox. Wildlife Research, 36: 106-109.

Valeix, M., Loveridge, A. J., Chamaillé-Jammes, S., Davidson, Z., Murindagomo, F.,Fritz, H. \& MacDonald, D.W. (2009). Behavioural adjustments of African herbivores to predation risk by lions: spatiotemporal variations influence habitat use. Ecology, 90: 23-30.

Valeix, M., Loveridge, A.J., Davidson, Z., Madzikanda, H., Fritz, H. \& Macdonald, D.W. (2010) How key habitat features influence large terrestrial carnivore movements: waterholes and African lions in a semi-arid savannah of north-western Zimbabwe. Landscape Ecology, 25: 337-351.

Valencak, T.G., Tataruch, F. \& Ruf, T. (2009) Peak energy turnover in lactating European hares: the role of fat reserves. Journal of Experimental Biology, 212: 231-237.

Vance-Chalcraft, H.D. \& Soluk, D.A. (2005) Estimating the prevalence and strength of non-independent predator effects. Oecologia, 146: 452-460.

Van der Stap, I., Vos, M., Verschoor, A.M., Helmsing, N.R. \& Mooij, W.M. (2007) Induced defences in herbivores and plants differentially modulate a trophic cascade. Ecology, 88(10): 2474-2481.

Van Dievel, M., Janssens, L. \& Stoks, R. (2015) Short- and long-term behavioural, physiological and stoichiometric responses to predation risk indicate chronic stress and compensatory mechanisms. Oecologia: DOI 10.1007/s00442-015-3440-1.

Van Vuuren, D. \& Coblentz, B.E. (1985) Kidney weight variation and the kidney fat index: an evaluation. The Journal of Wildlife Management, 49(1): 177-179.

Van Wieren, S.E., Wiersma, M. \& Prins, H. (2006) Climatic factors affecting a brown hare (Lepus europaeus) population. Lutra, 49: 103-110. 


\section{Bibliography}

Verdolin, J.L. (2006) Meta-analysis of foraging and predation risk trade-offs in terrestrial systems. Behavioural Ecology and Sociobiology, 60: 457-464.

Vijayan, S., Morris, D.W. \& McLaren, B.E. (2012) Prey habitat selection under shared predation: tradeoffs between risk and competition? Oikos, 121: 783-789.

Vogelbescherming (2017) Species information on their presence in the Netherlands during the year. https:// www.vogelbescherming.nl/ [accessed 19-2-2017].

Vucetich, J.A., Smith, D.W. \& Stahler, D.R. (2005) Influence of harvest, climate and wolf predation on Yellowstone elk, 1961-2004. Oikos, 111: 259-270.

Waggett, R.J. \& Buskey, E.J. (2007) Calanoid copepod escape behaviour in response to a visual predator. Marine Biology, 150: 599-607.

Weterings, M.J.A., Zaccaroni, M., Van der Koore, N., Zijlstra, L.M., Kuipers, H.J., Van Langevelde, F. \& Van Wieren, S.E. (2016) Strong reactive movement response of the medium-sized European hare to elevated predation risk in short vegetation. Animal Behaviour, 115: 107-114.

Whinam, J., Fitzgerald, N., Visoiu, M. \& Copson, G. (2014) Thirty years of vegetation dynamics in response to a fluctuating rabbit population on sub-Antarctic Macquarie Island. Ecological Management \& Restoration, 15(1): 41-51.

White, P.J. \& Garrott, R.A. (2005) Yellowstone's ungulates after wolves - expectations, realizations, and predictions. Biological Conservation, 125: 141-152.

White, P.J., Garrott, R.A., Hamlin, K.L., Cook, R.C., Cook, J.G. \& Cunningham, J.A. (2011) Body condition and pregnancy in northern Yellowstone elk: evidence for predation risk effects? Ecological Applications, 21(1): 3-8.

Wilkenros, C., Kuijper, D.J.P., Behnke, R. \& Schmidt, K. (2015) Behavioural responses of ungulates to indirect cues of an ambush predator. Behaviour, 152: 1019-1040.

Wilmshurst, J.F., Fryxell, J.M. \& Bergman, C.M. (2000) The allometry of patch selection in ruminants. Proceedings: Biological Sciences, 267(1441): 345-349.

Wincentz-Jensen, T.L. (2009) Identifying caused of population decline of the brown hare in agriculture landscapes in Denmark. PhD thesis. Aarhus, Denmark: National environment research institute, Aarhus University.

Wirsing, A.J., Cameron, K.E. \& Heithaus, M.R. (2010) Spatial responses to predators vary with prey escape mode. Animal Behaviour, 79: 531-537.

Wright, V.L. (1978) Causes and effects of biases on waterfowl harvest estimates. The Journal of Wildlife Management, 42(2): 251-262.

Ydenberg, R.C. \& Dill, L.M. (1986) The economics of fleeing from predators. Advances in the Study of Behaviour, 16: 229-249.

Ylönen, H., Jedrzejewki, B., Jedrzejewki, W. \& Heikkilä, J. (1992) Antipredator behaviour of Clethrionomy voles: 'David and Goliath' arms race. Annales Zoologici Fennici, 29:207-216.

Young, J.K., Olson, K.A., Reading, R.P., Amgalanbaatar, S. \& Berger, J. (2011) Is wildlife going to the dogs? Impacts of feral and free-roaming dogs on wildlife populations. BioScience, 61: 125-132.

Zanette, L.Y., Clinchy, M. \& Suraci, J.P. (2014) Diagnosing predation risk effects on demography: can measuring physiology provide the means. Oecologia, 176: 637-651.

Zanette, L.Y., Hobson, K.A., Clinchy, M., Travers, M. \& Williams, T.D. (2013) Food use is affected by the experience of nest predation: implications for indirect predator effects on clutch size. Oecologia, 172: 1031-1039.

Zanette, L.Y., White, A.F., Allen, M.C. \& Clinchy, M. (2011) Perceived predation risk reduces the number of offspring songbirds produced per year. Science, 334: 1398-1401.

Zuur, A.F., Ieno, E.N. \& Elphick, C.S. (2010) A protocol for data exploration to avoid common statistical problems. Methods in Ecology and Evolution, 1: 3-14. 




\section{Summary}



The relative importance and the role of predation risk for prey is still subject of debate, especially the relative strength of top-down versus bottom-up processes. European hare are thought to respond particularly strong to predation risk, which possibly contributed to the decline in population numbers in north-west Europe. Therefore the objective of this thesis was to investigate the effects of predation risk and habitat characteristics on European hare.

On a predator-free island, I subjected European hares to the elevated predation risk of a leashed dog, and tested how habitat characteristics affected the movement response of hares (chapter 2). Movement responses of GPS-collared hares were measured in patches with different vegetation structure on a short (i.e., several hours) and a long (i.e., 24 hours) timescale. The movement response of European hare to elevated predation risk was best explained by a model that included the interaction between predation risk and vegetation structure. On the short time scale, a strong immediate movement response was found in open habitat with low cover. However, on the long timescale, the effect of the treatment synchronized with the daily rhythm of the hare. The distance covered between resting and foraging grounds was negatively affected by elevated predation risk, while use of less risky (often low-quality) vegetation during resting and foraging was favoured.

Secondly, I tested the effects of predators (i.e., red fox (Vulpes vulpes)), fellow prey and habitat characteristics on the space use of two sympatric prey species, the European hare and European rabbit (Oryctolagus cuniculus) using camera traps (chapter 3). The response of hare and rabbit to space use by predators, space use by fellow prey, and habitat characteristics was different for both prey species investigated. The level of activity by rabbits, which make use of a refuge for escape (i.e., central-place forager), was positively correlated with the level of activity by predators over time, however, space use of rabbits was uncorrelated to space use by predators. The level of activity by hares, which do not make use of a refuge (i.e., free-ranging), was uncorrelated with the level of activity by predators over time, however, space use of hares was correlated with space use by predators. Predator absence possibly led to avoidance behaviour between hare and rabbit, while predator presence promoted coexistence between the two species. Competition for predator free space may have reversed the relationship between the space use by predators and habitat characteristics on the space use by hare and rabbit.

In chapter 4, I tested the relative importance of predator (fox) and competitor (rabbit) activity and forage quality and quantity for the proportion of time spent in a vegetation type, and the proportion of time spent foraging by European hare. Space use and foraging behaviour was investigated by equipping hares with 
GPS and accelerometers. Hand-plucked samples of plant species were used to analyse forage quality and quantity. The activity of predators and competitors was investigated using camera traps. During days that predators were more active, hares spent a higher proportion of time in low-risk patches, and in patches with low-quality food and low-quantity food. Overall, habitat characteristics (i.e., forage quality and vegetation height) more strongly affected hare space use than the activity of predators. The activity of competitors was not related to space use. During days that competitors were more active, hares spent a higher proportion of time foraging in patches of low-quality food. Overall, habitat characteristics (i.e., forage quality, vegetation height and edible biomass) more strongly affected hare foraging time than the activity of predators, and the activity of competitors was least important.

In chapter 5, I tested the correlation between chronic exposure to predation risk of multiple predators and body condition and reproductive output of European hare. Density of all predators year-round present was estimated. To describe body condition, I extracted four components of the body measurements of shot hares by a principal component analysis. Reproductive output of hares was estimated as the number of placental scars. Predation risk was negatively correlated with the number of placental scars and the 'health' component of body condition of European hare (i.e., weight of liver, kidney, heart and body).

In this thesis I showed that both bottom-up and top-down forces modulate prey species behaviour and distribution simultaneously. Overall, predators seemed less important than habitat characteristics in affecting space use and foraging behaviour on the scale of the food patch and the scale of the daily range. Nevertheless, the effect of predators per se on space use and foraging behaviour was more important at the scale of the food patch than at the scale of the daily range. My conclusion is that the relative importance of predators for the behavioural response of prey is negatively correlated with the scale of the processes. Additionally, predators seemed fairly important in affecting body condition and reproductive output at the scale of the home range. I expect the relative importance of predators for prey fitness to be positively related with the scale of the processes. Insight into the effects of predation risk and habitat characteristics on European hare, helps us to understand the responses of hares to the changes in the north-west European landscape. The increased numbers, accessibility and distribution of predators has probably contributed to the decline in population numbers of the European hare in north-west Europe. 




\section{Samenvatting}



Het relatieve belang en de rol van predatierisico voor prooidieren is nog steeds onderwerp van discussie, met name het relatieve belang van top-down versus bottom-up processen. Van Europese hazen wordt verondersteld dat ze bijzonder sterk reageren op predatierisico, wat mogelijk heeft bijgedragen aan de achteruitgang van de populatie in noordwest Europa. Het doel van dit proefschrift is inzicht te krijgen in de effecten van predatierisico en habitatkenmerken op de Europese haas.

Op een eiland zonder roofdieren onderwierp ik Europese hazen aan een verhoogd predatierisico van een aangelijnde hond, en testte ik hoe habitatkenmerken de bewegingsreactie van hazen beïnvloedden (hoofdstuk 2). De bewegingsreacties van GPS-gezenderde hazen werden gemeten in verschillende vegetatiestructuren op een korte (d.w.z. verscheidene uren) en een lange (d.w.z. 24 uur) tijdschaal. De reactie van hazen op een verhoogd predatierisico werd het best verklaard door een model met een interactie tussen predatierisico en vegetatiestructuur. Op een korte tijdschaal werd een sterke acute reactie gemeten in open habitat met een lage dekking. Echter, op een lange tijdschaal, volgde het effect van de behandeling het dagritme van de haas. Een verhoogd predatierisico had een negatief effect op de afgelegde afstand tussen rust- en foerageergebieden. Daarnaast hadden hazen tijdens het rusten en het foerageren een voorkeur voor minder risicovolle (vaak lage kwaliteit) vegetatie.

In een tweede project heb ik met behulp van cameravallen onderzoek gedaan naar de effecten van roofdieren (d.w.z. Vos (Vulpes vulpes)), mede-prooisoorten en habitatkenmerken op het ruimtegebruik van twee prooisoorten, de Europese haas en het Europese konijn (Oryctolagus cuniculus) (hoofdstuk 3). De reactie van haas en konijn op het ruimtegebruik door roofdieren, het ruimtegebruik door mede-prooidiersoorten en habitatkenmerken was verschillend voor beide prooidiersoorten. De mate van activiteit van konijnen, die gebruik maken van een burcht als toevluchtsoord om te ontsnappen, was positief gecorreleerd met de mate van activiteit van roofdieren in de tijd. Het ruimtegebruik door konijnen was echter niet gecorreleerd met het ruimtegebruik door roofdieren. De mate van activiteit van hazen, die geen gebruik maken van een toevluchtsoord, was niet gecorreleerd met de mate van activiteit van roofdieren in de tijd. Het ruimtegebruik door hazen was echter wel gecorreleerd met het ruimtegebruik door roofdieren. De afwezigheid van roofdieren leidde mogelijk tot vermijdingsgedrag tussen haas en konijn, terwijl de aanwezigheid van roofdieren mogelijk de co-existentie tussen beide soorten bevorderde. Competitie tussen haas en konijn voor locaties zonder roofdieren heeft er mogelijk voor gezorgd dat de relatie tussen het ruimtegebruik door roofdieren en habitatkenmerken op het ruimtegebruik door haas en konijn werd omgekeerd. 
In hoofdstuk 4 heb ik het relatieve belang onderzocht van de mate van activiteit van roofdieren (vos) en concurrenten (konijn) en de kwaliteit en kwantiteit van voedsel, voor de relatieve tijd die hazen doorbrachten in een vegetatietype, en de relatieve tijd die hazen besteedden aan foerageren. Ruimtegebruik en foerageergedrag werden onderzocht door hazen te voorzien van GPS en versnellingsmeters. Met de hand geplukte samples van plantensoorten werden gebruikt om de kwaliteit en kwantiteit van het voedsel te analyseren. De mate van activiteit van roofdieren en concurrenten werd onderzocht met behulp van cameravallen. Tijdens dagen dat roofdieren actiever waren, brachten hazen een groter deel van de tijd door in vegetatietypen met een laag risico op predatie, in vegetatietypen met weinig voedsel, en in vegetatietypen met voedsel van lage kwaliteit. Over het algemeen waren de habitatkenmerken (d.w.z. voedselkwaliteit en vegetatiehoogte) sterker gerelateerd aan het ruimtegebruik door hazen dan de mate van activiteit van roofdieren. De mate van activiteit van concurrenten was niet gerelateerd aan het ruimtegebruik door hazen, maar was wel positief gerelateerd aan de fractie van de tijd dat hazen foerageerden in vegetatietypen met een lage voedsel kwaliteit. Over het geheel genomen beïnvloedden de habitatkarakteristieken (d.w.z. voedselkwaliteit, vegetatiehoogte en de hoeveelheid eetbare biomassa) de foerageertijd van hazen sterker dan de mate van activiteit van roofdieren, waarbij de mate van activiteit van concurrenten het minst sterk gerelateerd was.

In hoofdstuk 5 heb ik de correlatie getest tussen de chronische blootstelling aan het predatierisico van meerdere roofdieren, de lichaamsconditie en het voortplantingssucces van de Europese haas. De dichtheid van alle roofdieren die het hele jaar door aanwezig waren werd geschat door jagers. Om de lichaamsconditie te beschrijven, heb ik de metingen aan het lichaam van hazen in vier hoofdcomponenten gekwantificeerd door middel van een 'Principal Component Analysis'. Het voortplantingssucces van hazen werd geschat door het aantal littekens van voormalige placenta's te tellen. Het predatierisico was negatief gecorreleerd met het aantal littekens van voormalige placenta's en de 'gezondheids'-component van de lichaamsconditie van Europese hazen (d.w.z. het gewicht van de lever, de nieren, het hart en het lichaam).

In dit proefschrift heb ik angetoond dat zowel bottom-up als top-down processen tegelijkertijd het gedrag en de verspreiding van prooidieren beïnvloedden. Over het algemeen leken roofdieren minder belangrijk dan habitatkenmerken in het beïnvloeden van het ruimtegebruik en het foerageergedrag van hazen op een kleine (foerageerplek) en middelgrote (dagbesteding) schaal. Desalniettemin, op een kleine schaal was het effect van roofdieren op het ruimtegebruik en het foerageergedrag van hazen sterker dan op een middelgrote schaal. Mijn conclusie is dat het relatieve belang van roofdieren voor het ruimtegebruik en het foera- 
geergedrag van prooidieren sterker wordt op een kleiner schaalniveau. Roofdieren bleken bovendien tamelijk belangrijk in het beïnvloeden van de lichaamsconditie en het voortplantingssucces op grotere schaal (leefgebied). Ik verwacht dat het relatieve belang van roofdieren voor het voortplantingssucces sterker wordt op een groter schaalniveau. Inzicht in de effecten van predatierisico en habitatkenmerken op de Europese haas helpt ons de reacties van hazen op de veranderingen in het Noordwest-Europese landschap beter te begrijpen. De toename van de aantallen roofdieren, hun toegankelijkheid, en hun distributie heeft waarschijnlijk bijgedragen aan de achteruitgang van de populatie aantallen van de noordwest Europese haas. 



\section{Acknowledgements}



I would not have been able to successfully complete my doctorate without the support and help of many people.

Suzanne, I am especially indebted to you and grateful for your support. You managed to stay relaxed and sedate during the many hectic months that we encountered during my doctorate. Above all, you were especially able to complement, and take over my household activities and the upbringing of our children (Jasmijn and Lente) at the right time, in addition to carrying out your own work. You have my utmost respect for that. You also never complained about this crazy project that I started and really enjoyed up to the end.

Sip, I am very grateful for the trust you invested in me from the start of this project. I have learned a lot from your enormous mental library of real field knowledge regarding all aspects of ecology, i.e., plant and animal species, in the temperate zone. I have always enjoyed our discussions during my doctorate and especially during the times we wrote papers together. Most of the times you magically conjured up some dark musty manuscript from the bookshelf or some obscure box that could aid in the defence of our data. Besides that, I can still remember many of the nice moments we had together, such as the lively discussions about nature management in the Netherlands, eating pizzas and drinking beer on Schiermonnikoog and Wageningen, or capturing hares in the freezing cold.

Frank, we laughed a lot during my doctorate, especially because we were often making jokes about each other or about your hair. Luckily we were both not offended by it. I think you need to make jokes to compensate for your hard working. Besides that, I really enjoyed our time discussing papers and other matters. I am grateful for the trust and freedom you gave me. You were also irreplaceable when it comes to teaching me how to write a paper and handle the reasoning behind. However, even with your advice, I rewrote our camera paper about twenty times, and we still can't find a publisher that is willing to even look at the paper. Probably that is because of my pig-headedness. Besides reasoning, you were very knowledgeable about research, as well as statistics, and I have learned a lot from it. Hopefully our next project will be successful and fun too.

Herbert, I am very happy that I was allowed to do my doctorate in your department. I always felt welcome and you always supported me throughout the process. During the breaks, I enjoyed your discussions about the craziest subjects that often led to a surprising insight with your skilled way of argumentation. I really appreciated the freedom you gave me to work on my topic. You also ignited the writing of a SIA RAAK proposal that led to the successful funding of my new project. Thank you for that. 
Theo, I would not have been able to complete this research successfully without you. Because of your flexibility and understanding I could always make time during the regular education for my doctorate study and the many meetings that were always planned during my lectures. Additionally, you were (as always) enthusiastic about (hare) research yourselves, scientifically challenging, knowledgeable and well informed about every topic that is currently in the news (and was in the news earlier). Even though I was not able to follow your footsteps every time, I am grateful for all the things you have learned me throughout my career. Besides, you were great to collaborate with, and I appreciated your keen feedback on the introduction and the general discussion. I have only one advice to you: eat more chocolate!

Henry, I have great admiration for your knowledge, expertise and professionalism as a colleague, but especially with regard to statistics and research. At times when no other statistician was able to help me (and that happened a lot), you came up with the right direction. I believe that I have asked you more than a thousand questions about the preferred statistical approach and the interpretation of the data and the results from my research. There was not a single time that you were not helpful, or that you were 'too busy' to be involved in my statistical problems. Above all, I like working with you. Our collaboration on various thesis projects was characterized by an open direct and honest attitude, which I am especially fond of. We are a good team.

I would like to express my special gratitude to the WM team: Arjen, Berend, Christa, Froukje, Hans, Henry, Ignas, Jelmer, Jeroen, Marcel, Miranda, Okka, Rick, Theo, Tine and Wimke for their great selfless job and limitless support, as they had to take over a lot of my former teaching work during my doctorate, so I was able to concentrate myself on working on my hare project. In recent years, they have done a huge amount of work in too little time, without taking a break. You are the best colleagues one could wish for.

Patrick, we have had many discussions about my camera trap research. Whenever I was struggling, you repeatedly took time to help me reorganising my thoughts. I really appreciated your feedback. When it comes to your ideas about how to structure the general idea of a paper, you are also outspoken and honest. I sometimes felt myself in the middle of a scientific debate between several people pointing towards different directions. I struggled with that, because most of the times I wanted to try to take the best of both worlds, however that did not always work out. I enjoyed your camera trap labs, as they stimulated my thoughts about various aspects of camera trapping research. Lastly, I also enjoyed our discussions about nature management in the Netherlands. Besides being a scientist, you are a true political animal. 
Klaus, I would like to thank you for the general advice you gave me during my work. In particular Suzanne and I enjoyed our stay at your laboratory in Vienna, we felt very welcome and you selflessly spent a lot of time teaching us how to count placental scars. Moreover, I enjoyed the moments when we met, such as during the Lagomorph conferences. The $4^{\text {th }}$ Lagomorph Conference in Vienna was fantastic!

Dries, it is a pity that we did not meet during my doctorate, however you consistently provided constructive feedback quickly and whenever necessary. Thank you for that.

Tim, we discussed various aspects of our research during the five years that we were roommates. I always admired your knowledge of natural history and high standard of doing research. I am sure that you will end up as one of the leading world scientists in your field. Thanks for your pleasant company.

From my employer, the Van Hall Larenstein University of Applied Sciences, this would not have been possible without the strong unconditional support of Hans Hardus (the program manager at that time), Tjalling Huisman (the present program manager) and Diane Keizer and Peter van Dongen (the Executive Board), as they have greatly facilitated my project by ensuring that all sorts of nasty organizational problems were greatly reduced.

Joost, thanks for your feedback on my introduction and discussion. Lennart and Claudius (the PE\&RC team), thanks for the wonderful courses, meetings and discussions you organised and offered. They are of genuine added value to my career as a scientist.

In particular, I have enjoyed my stay at the Resource Ecology Group, and would like to thank all my fellow $\mathrm{PhD}$ students and the staff for the fantastic scientific and non-scientific atmosphere.

Lastly, Erik, Koen and Suzanne, thanks for your feedback on the thesis booklet in general, and Erik and Koen for being my paranymphs.

A lot of people and organisations helped me during the fieldwork of one of my chapters.

Chapter 2: I am indebted to Danny Brandwacht and Jelle Hazenbosch, who assisted in the field experiment, Martijn van der Ende, Nel van Vliet, Diva the retriever, many enthusiastic students from the VHL department of Wildlife Management and the WUR Resource Ecology Group for field assistance, and Natuurmonumenten for providing access to the study area and logistical support.

Chapter 3 \& 4: I am indebted to Bart Albers, Lisa van Erp, Kyra Fastenau, Rick Heeres, Inger de Jonge, Jingyue Jongsma, Julia Kestler, Theo Meijer, Sander Moonen, Robert Oudejans, Kira Rokx, David Smit, Nina Schouten, Arjen Strijkstra, Martijn Struijf, Sharon Boekhout and Martijn van der Ende for field 
assistance, and Jan van Walsem for lab assistance with plant nutrient analysis. I thank PWN 'Waterleidingbedrijf Noord-Holland', for providing access to the study area, and; Hubert Kivit, Paul van der Linden, Dave Nanne and Evert-Jan Woudsma from PWN for logistical support.

Chapter 5: I am indebted to Arjen Strijkstra, Sophie Bader, Christina Cappello, Greta Dekker, Rieke Hochstrat, Sven Kolbe, Jonno Stelder, and Aron Witzand for lab assistance, Gerrit Gort for assistance with statistical analysis and Klaus Hackländer for assistance with placental scar counts. I thank the KJV and their local hunting groups for providing access to their hunting leases, assistance in the questionnaire and for providing the samples. I thank the Dutch Wildlife Health Centre for examining tissue samples for general pathology. 




\section{Short biography}



Martinus Jacobus Antonius Weterings was born on the $18^{\text {th }}$ of September 1970 in Oosterhout, the Netherlands. He is fascinated by history, old paintings, indigenous art and objects, reptiles and amphibians, and likes hiking, camping, running and canoeing. After completing mavo, havo and vwo, he chose to study aerospace industry technology at the Technical University of Delft from 19901993. In 1994, in pursuit of more excitement, he switched his study, but was obligated to fulfil military service first. After working for the UN peacekeeping mission (UNPROFOR) in Bosnia and Herzegovina mid-1994, he started to study Wildlife Management at the Van Hall Larenstein University of Applied Sciences in Groningen and later in Leeuwarden. During his study, he travelled to Thailand, Taiwan and Panama to do field research on primates. His BSc thesis (cum laude) focussed on a population viability assessment for the European otter (Lutra lutra) that was successfully reintroduced in the Netherlands later in 2002. In 1999-2000 he followed an MSc in Applied Ecology and Conservation at the University of East Anglia. For his MSc thesis (with distinction), he went to Surinam to study spider monkey foraging ecology in the Raleighvallen Voltzberg Nature Reserve. After returning from the UK he accepted a teaching position at the Van Hall Larenstein (VHL) in wildlife management ecology and research techniques. In 2009 and 2012, he and Suzanne were lucky to get two beautiful children ('Jasmijn' and 'Lente'). In 2011 he obtained a personal doctoral grant for teachers from the Netherlands Organisation of Scientific Research (NWO) and started his doctoral study in August 2012 at Wageningen University. During his doctoral study he was strongly involved in a RAAK Pro application from SIA (NWO) that was honoured in 2017. Currently, besides teaching at the VHL, he is working as a program manager and scientist at the professorship 'animal behaviour, health and welfare' and as a postdoctoral researcher at the Resource Ecology Group, Wageningen University. Together with a strong consortium (WUR, SAXION, SODAQ and WNF), he aims to develop a sensor network that captures the alarm responses of ungulate herds to prevent poaching of endangered megafauna. 



\section{Publications}





\section{PUBLISHED ARTICLES}

Rademaker, M., Rode-Margono, E.J. \& Weterings, M. (2017) Estimation of species area abundance from point abundance data, using effective detection areas from camera traps. Journal of Biodiversity and Endangered Species, 5: 200. DOI:10.4172/2332-2543.1000200

Weterings, M.J.A., Zaccaroni, M., Van der Koore, N., Zijlstra, L.M., Kuipers, H.J., Van Langevelde, F. \& Van Wieren, S.E. (2016) Strong reactive movement response of the medium-sized European hare to elevated predation risk in short vegetation. Animal Behaviour, 115: 107-114.

Stark, T., Laurijssens, C., Weterings, M., Martel, A., Köhler, G. \& Pasmans, F. (2016) Prevalence of Batrachochytrium dendrobatidis in a Nicaraguan, micro-endemic Neotropical salamander, Bolitoglossa mombachoensis. Amphibia-Reptilia: 1-6. DOI:10.1163/15685381-00003077

Loehr, V.J.T., Stark, T., Weterings, M. \& Kuijpers, H. (2015) Overcoming low environmental temperatures in the primary feeding season: low-level activity and long basking in the tortoise Homopus signatus. Amphibia-Reptilia: 1-8. DOI:10.1163/15685381-00002994

Raap, T., Van Schoote, G., Van Dieren, M., Hedger, C., Kuipers, H. \& Weterings, M. (2015) The effect of fire on the habitat use of the Black-eared Miner Manorina melanotis. Ardea, 103(1): 79-90.

Linden, V.M.G., Weier, S.M., Gaigher, I., Kuipers, H.J., Weterings, M.J.A. \& Taylor, P. (2014) Changes of bat activity, species richness, diversity and community composition over an altitudinal gradient in the Soutpansberg range, South Africa. Acta Chiropterologica, 16(1): 27-40.

Stark, T., Laurijssens, C. \& Weterings, M. (2014) Distributional and natural history notes on five species of amphibians and reptiles from Isla Ometepe, Nicaragua. Mesoamerican Herpetology, 1(2): 308-312.

Stark, T., Laurijssens, C., Weterings, M., Spitzen-van der Sluijs, A., Martel, A. \& Pasmans, F. (2014) Death in the clouds: ranavirus associated mortality in assemblage of cloud forest amphibians in Nicaragua. Acta Herpetologica, 9(1): 125-127. 
Koschnicke, S., Franke, L., Doormaal, F. van, Kuipers, H., Weterings, M., GlozaRausch, F. \& Konadu-Ameyaw, J. (2010) Bat boxes as a tool for biological insect pest control on cocoa plantations in Ghana. Nyctalus, 15(4): 357-366.

De Pous, P., Beukema, W., Weterings, M., Dümmer, I. \& Geniez, P. (2010) Area prioritization and performance evaluation of the conservation area network for the Moroccan herpetofauna: a preliminary assessment. Biodiversity Conservation (DOI 10.1007/s10531-010-9948-0)

Weterings, M.J.A., Schonck, S.M., Vester, H.F.M. \& Calme, S. (2008) Senescence of Manilkara zapota trees and implications for large frugivorous birds in the Southern Yucatan Peninsula, Mexico. Forest Ecology and Management, 256(9): 1604-1611.

\section{IN PREPARATION}

Weterings, M.J.A., Ewert, S.P., Peereboom, J.N., Kuipers, H.J., Kuijper, D.P.J., Prins, H.H.T., Jansen, P.A., Van Langevelde, F. \& Van Wieren, S.E. Implications of apparent competition for the space use of two sympatric leporids.

Weterings, M.J.A., Losekoot, S., Kuipers, H.J., Prins, H.H.T., Van Langevelde, F. \& Van Wieren, S.E. Chronic exposure to high predation risk by multiple predators decreases body condition and reproductive fertility of prey.

Weterings, M.J.A., Moonen, S., Prins, H.H.T., Van Wieren, S.E. \& Van Langevelde, F. Food quality and quantity is more important explaining foraging of an intermediate-sized mammalian herbivore than predation or competition 




\section{Affiliation of co-authors}



Sipke E. van Wieren, Frank van Langevelde, Herbert H.T. Prins, Patrick A. Jansen: Resource Ecology Group, Department of Environmental Sciences, Wageningen University, Wageningen the Netherlands

Henry J. Kuipers, Sophie P. Ewert, Nikki van der Koore, Sanne Losekoot, Sander Moonen, Jeffrey N. Peereboom, Linda M. Zijlstra:

Wildlife Management, Department of Animal Management, Van Hall Larenstein University of Applied Sciences, Leeuwarden, the Netherlands

Dries P.J. Kuijper:

Population Ecology Unit, Mammal Research Institute, Polish Academy of Sciences, Białowieża, Poland.

Marco Zaccaroni:

Department of Biology, University of Florence, Sesto Florence, Italy 



\section{PE\&RC Training and Education}

\section{Statement}



With the training and education activities listed below the $\mathrm{PhD}$ candidate has complied with the requirements set by the C.T. de Wit Graduate School for Production Ecology and Resource Conservation (PE\&RC) which comprises of a minimum total of 32 ECTS (= 22 weeks of activities)

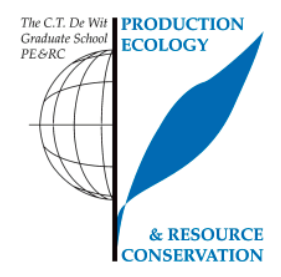

\section{Review of literature (4.5 ECTS)}

- Declining European hare (Lepus europaeus) populations as consequence of effects of habitat heterogeneity, diet quality and indirect effects of predation

\section{Writing of project proposal (4.5 ECTS)}

- European hare (Lepus europaeus) population reproduction as a resultant of landscape quality: effects of habitat heterogeneity, diet quality and indirect effects of predation

\section{Post-graduate courses (5.7 ECTS)}

- Multivariate Analysis; PE\&RC (2012)

- Bayesian Statistics; PE\&RC (2012)

- Generalized Linear Models; PE\&RC (2013)

- Mixed Linear Models; PE\&RC (2013)

- Spatial Ecology; PE\&RC (2014)

\section{Laboratory training and working visits (0.9 ECTS)}

- Identification of placental scars in European hare uteri; BOKU, Vienna (2014)

\section{Invited review of (unpublished) journal manuscript (2 ECTS)}

- Mammal Review: 'Flexibility in the food selection by the brown hare (Lepus europaeus) along the altitudinal gradient of Southern Andean Precordillera (Argentina)'

- Animal Behaviour: 'Different responses to predation risk of native and invasive rodents in a tropical savannah'

\section{Competence strengthening / skills courses (2.5 ECTS)}

- Scientific publishing; WGS (2012)

- Communication in interdisciplinary research; WGS (2012)

- Reviewing a scientific paper; WGS (2013)

- Techniques for writing and presenting a scientific paper; WGS (2016) 
PE\&RC Annual meetings, seminars and the PE\&RC weekend (2.4 ECTS)

- PE\&RC Weekend (2013)

- PE\&RC Day (2013, 2014, 2015)

- Current Themes in Ecology (NERN) (2014, 2017)

Discussion groups / local seminars / other scientific meetings (4.1 ECTS)

- WEES (2012, 2013, 2015, 2016)

- Field meeting European hare netting \& tagging (2012, 2014)

- Ecological theory and application (disc. Propositions) (2013)

- FEM Guest lecture (2015)

- Camera trapping lab $(2016,2017)$

\section{International symposia, workshops and conferences (10.8 ECTS)}

- Annual meeting of ATBC $50^{\text {th }}$ anniversary; poster presentation

- $\quad 4^{\text {th }}$ World Lagomorph Conference; poster presentation

- $8^{\text {th }}$ EU Zoo Nutrition Conference EAZA; poster presentation

- $\quad 5^{\text {th }}$ World Lagomorph Conference; oral presentation and poster presentation

- Predator-Prey interactions GRC Conference; poster presentation

\section{Lecturing / Supervision of practicals / tutorials (6 ECTS)}

- The ecological advisor; Van Hall Larenstein University of Applied Sciences, Leeuwarden (2012-2018)

- Introduction nature management; Van Hall Larenstein University of Applied Sciences, Leeuwarden (2012-2018)

\section{Supervision of 4 MSc students (12 ECTS)}

- Hare behavioural ecology Schiermonnikoog 2x

- Hare foraging ecology Castricum

- Hare response to risk effects (sounds) Castricum 
The research described in this thesis was financially supported by the Netherlands Organisation for Scientific Research (NWO) Doctoral Grant for Teachers (023.001.222), the Van Hall Larenstein University of Applied Sciences, and Wageningen University.

Financial support from Wageningen University for printing this thesis is gratefully acknowledged.

Cover design: Martijn Weterings

Lay-out and print by: ProefschriftMaken // www.proefschriftmaken.nl 


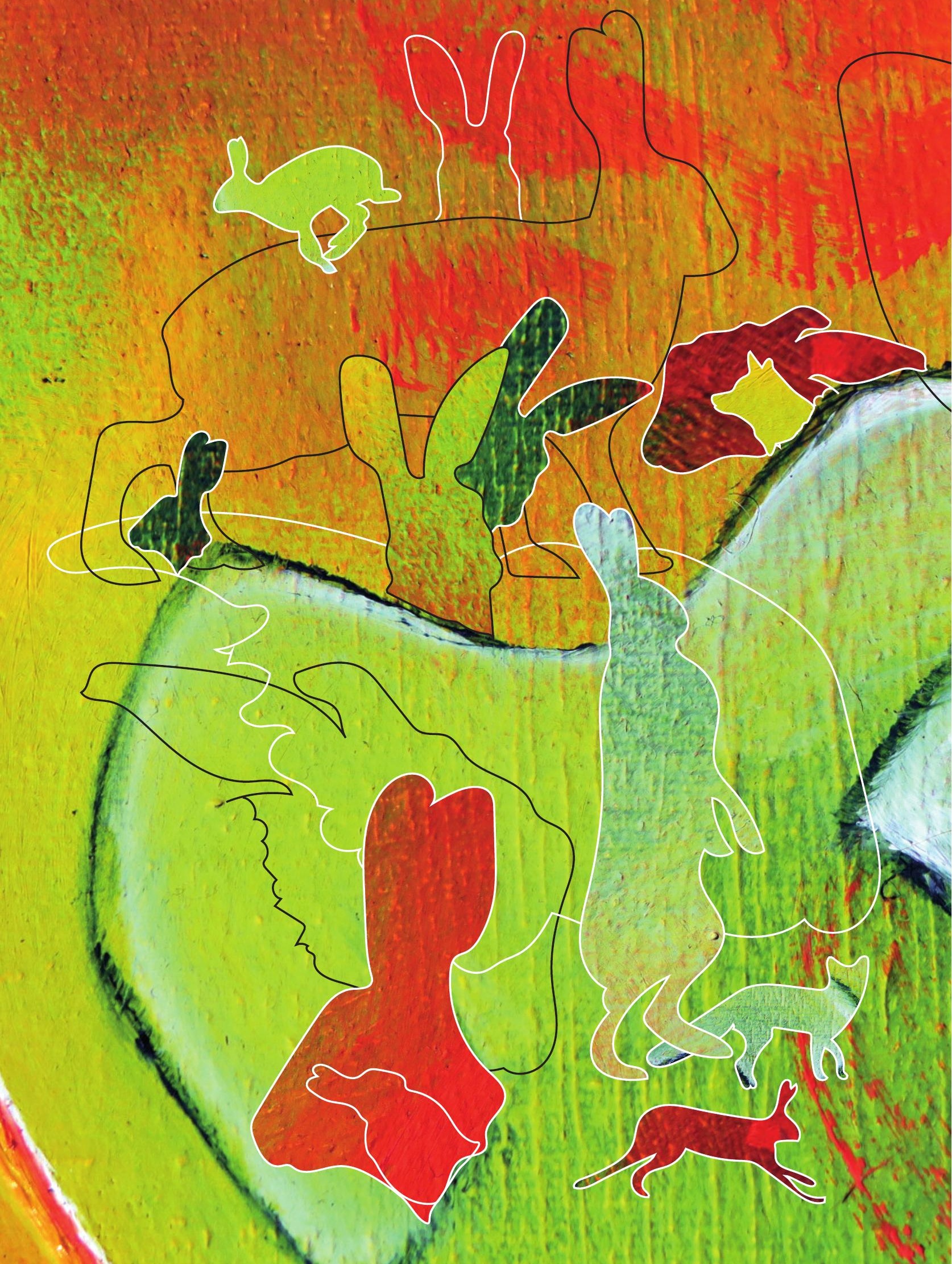

